\title{
GEMPIC: geometric electromagnetic particle-in-cell methods
}

\author{
Michael Kraus ${ }^{1,2,}$, , Katharina Kormann ${ }^{1,2}$, Philip J. Morrison ${ }^{3}$ \\ and Eric Sonnendrücker ${ }^{1,2}$ \\ ${ }^{1}$ Max-Planck-Institut für Plasmaphysik, Boltzmannstraße 2, 85748 Garching, Deutschland \\ ${ }^{2}$ Technische Universität München, Zentrum Mathematik, Boltzmannstraße 3, \\ 85748 Garching, Deutschland \\ ${ }^{3}$ Department of Physics and Institute for Fusion Studies, The University of Texas at Austin, \\ Austin, TX 78712, USA
}

(Received 20 January 2017; revised 12 May 2017; accepted 12 May 2017)

We present a novel framework for finite element particle-in-cell methods based on the discretization of the underlying Hamiltonian structure of the Vlasov-Maxwell system. We derive a semi-discrete Poisson bracket, which retains the defining properties of a bracket, anti-symmetry and the Jacobi identity, as well as conservation of its Casimir invariants, implying that the semi-discrete system is still a Hamiltonian system. In order to obtain a fully discrete Poisson integrator, the semi-discrete bracket is used in conjunction with Hamiltonian splitting methods for integration in time. Techniques from finite element exterior calculus ensure conservation of the divergence of the magnetic field and Gauss' law as well as stability of the field solver. The resulting methods are gauge invariant, feature exact charge conservation and show excellent long-time energy and momentum behaviour. Due to the generality of our framework, these conservation properties are guaranteed independently of a particular choice of the finite element basis, as long as the corresponding finite element spaces satisfy certain compatibility conditions.

Key words: magnetized plasmas, plasma nonlinear phenomena, plasma simulation

\section{Introduction}

We consider a structure-preserving numerical implementation of the VlasovMaxwell system, which is a system of kinetic equations describing the dynamics of charged particles in a plasma, coupled to Maxwell's equations, describing electrodynamic phenomena arising from the motion of the particles as well as from externally applied fields. While the design of numerical methods for the Vlasov-Maxwell (and Vlasov-Poisson) system has attracted considerable attention since the early 1960s (see Sonnendrücker 2017 and references therein), the systematic development of structure-preserving or geometric numerical methods started only recently.

$\dagger$ Email address for correspondence: michael.kraus@ipp.mpg.de 
The Vlasov-Maxwell system exhibits a rather large set of structural properties, which should be considered in the discretization. Most prominently, the VlasovMaxwell system features a variational (Low 1958; Ye \& Morrison 1992; Cendra et al. 1998) as well as a Hamiltonian (Morrison 1980; Weinstein \& Morrison 1981; Marsden \& Weinstein 1982; Morrison 1982) structure. This implies a range of conserved quantities, which by Noether's theorem are related to symmetries of the Lagrangian and the Hamiltonian, respectively. In addition, the degeneracy of the Poisson brackets in the Hamiltonian formulation implies the conservation of several families of so-called Casimir functionals (see e.g. Morrison 1998 for a review).

Maxwell's equations have a rich structure themselves. The various fields and potentials appearing in these equations are most naturally described as differential forms (Bossavit 1990; Baez \& Muniain 1994; Warnick, Selfridge \& Arnold 1998; Warnick \& Russer 2006) (see also Darling 1994; Morita 2001; Dray 2014). The spaces of these differential forms build what is called a deRham complex. This implies certain compatibility conditions between the spaces, essentially boiling down to the identities from vector calculus, curl grad $=0$ and $\operatorname{div}$ curl $=0$. It has been realized that it is of utmost importance to preserve this complex structure in the discretization in order to obtain stable numerical methods. This goes hand in hand with preserving two more structural properties provided by the constraints on the electromagnetic fields, namely that the divergence of the magnetic field $\boldsymbol{B}$ vanishes, $\operatorname{div} \boldsymbol{B}=0$, and Gauss' law, $\operatorname{div} \boldsymbol{E}=\rho$, stating that the divergence of the electromagnetic field $\boldsymbol{E}$ equals the charge density $\rho$.

The compatibility problems of discrete Vlasov-Maxwell solvers has been widely discussed in the particle-in-cell (PIC) literature (Eastwood 1991; Villasenor \& Buneman 1992; Esirkepov 2001; Umeda et al. 2003; Barthelmé \& Parzani 2005; Yu et al. 2013) for exact charge conservation. An alternative is to modify Maxwell's equations by adding Lagrange multipliers to relax the constraint (Boris 1970; Marder 1987; Langdon 1992; Munz et al. 1999, 2000). For a more geometric perspective on charge conservation based on Whitney forms one can refer to Moon, Teixeira \& Omelchenko (2015). Even though it has attracted less interest the problem also exists for grid-based discretizations of the Vlasov equations and the same recipes apply there as discussed in Sircombe \& Arber (2009), Crouseilles, Navaro \& Sonnendrücker (2014). Note also that the infinite-dimensional kernel of the curl operator has made it particularly hard to find good discretizations for Maxwell's equations, especially for the eigenvalue problem (Caorsi, Fernandes \& Raffetto 2000; Hesthaven \& Warburton 2004; Boffi 2006, 2010; Buffa and Perugia 2006).

Geometric Eulerian (grid-based) discretizations for the Vlasov-Poisson system have been proposed based on spline differential forms (Back \& Sonnendrücker 2014) as well as variational integrators (Kraus, Maj \& Sonnendruecker in preparation; Kraus 2013). While the former guarantees exact local conservation of important quantities like mass, momentum, energy and the $L^{2}$ norm of the distribution function after a semi-discretization in space, the latter retains these properties even after the discretization in time. Recently, also various discretizations based on discontinuous Galerkin methods have been proposed for both, the Vlasov-Poisson (de Dios, Carrillo \& Shu 2011, 2012; de Dios \& Hajian 2012; Heath et al. 2012; Cheng, Gamba \& Morrison 2013; Madaule, Restelli \& Sonnendrücker 2014) and the Vlasov-Maxwell system (Cheng, Christlieb \& Zhong 2014a,b; Cheng et al. 2014c). Even though these are usually not based on geometric principles, they tend to show good long-time conservation properties with respect to momentum and/or energy.

First attempts to obtain geometric semi-discretizations for particle-in-cell methods for the Vlasov-Maxwell system have been made by Lewis (1970, 1972). In his 
works, Lewis presents a fairly general framework for discretizing Low's Lagrangian (Low 1958) in space. After fixing the Coulomb gauge and applying a simple finite difference approximation to the fields, he obtains semi-discrete, energy and charge-conserving Euler-Lagrange equations. For integration in time the leapfrog method is used. In a similar way, Evstatiev, Shadwick and Stamm performed a variational semi-discretization of Low's Lagrangian in space, using standard finite difference and finite element discretizations of the fields and an explicit symplectic integrator in time (Evstatiev \& Shadwick 2013; Shadwick, Stamm \& Evstatiev 2014; Stamm \& Shadwick 2014). On the semi-discrete level, energy is conserved exactly but momentum and charge are only conserved in an average sense.

The first semi-discretization of the noncanonical Poisson bracket formulation of the Vlasov-Maxwell system (Morrison 1980; Weinstein \& Morrison 1981; Marsden \& Weinstein 1982; Morrison 1982) can be found in the work of Holloway (1996). Spatial discretizations based on Fourier-Galerkin, Fourier collocation and Legendre-Gauss-Lobatto collocation methods are considered. The semi-discrete system is automatically guaranteed to be gauge invariant as it is formulated in terms of the electromagnetic fields instead of the potentials. The different discretization approaches are shown to have varying properties regarding the conservation of momentum maps and Casimir invariants but none preserves the Jacobi identity. It was already noted by Morrison (1981a) and Scovel \& Weinstein (1994) however that grid-based discretizations of noncanonical Poisson brackets do not appear to inherit a Poisson structure from the continuous problem and Scovel \& Weinstein suggested that one should turn to particle-based discretizations instead. In fact, for the vorticity equation it was shown by Morrison (1981b) that using discrete vortices leads to a semi-discretization that retains the Hamiltonian structure. Such an integrator for the Vlasov-Ampère Poisson bracket was first presented by Evstatiev \& Shadwick (2013), based on a mixed semi-discretization in space, using particles for the distribution function and a grid-based discretization for the electromagnetic fields. However, this work lacks a proof of the Jacobi identity for the semi-discrete bracket, which is crucial for a Hamiltonian integrator.

The first fully discrete geometric particle-in-cell method for the Vlasov-Maxwell system has been proposed by Squire, Qin \& Tang (2012), applying a fully discrete action principle to Low's Lagrangian and discretizing the electromagnetic fields via discrete exterior calculus (DEC) Hirani (2003), Desbrun, Kanso \& Tong (2008), Stern et al. (2014). This leads to gauge-invariant variational integrators that satisfy exact charge conservation in addition to approximate energy conservation. Xiao et al. (2015) suggest a Hamiltonian discretization using Whitney form interpolants for the fields. Their integrator is obtained from a variational principle, so that the Jacobi identity is satisfied automatically. Moreover, the Whitney form interpolants preserve the deRham complex structure of the involved spaces, so that the algorithm is also charge conserving. Qin et al. (2016) use the same interpolants to directly discretize the canonical Vlasov-Maxwell bracket (Marsden \& Weinstein 1982) and integrate the resulting finite dimensional system with the symplectic Euler method. He et al. (2016) introduce a discretization of the noncanonical Vlasov-Maxwell bracket, based on first-order finite elements, which is a special case of our framework. The system of ordinary differential equations obtained from the semi-discrete bracket is integrated in time using the splitting method developed by Crouseilles, Einkemmer \& Faou (2015) with a correction provided by He et al. (2015) (see also Qin et al. 2015). The authors prove the Jacobi identity of the semi-discrete bracket but skip over the Casimir invariants, which also need to be conserved for the semi-discrete system to be Hamiltonian. 
In this work, we unify many of the preceding ideas in a general, flexible and rigorous framework based on finite element exterior calculus (FEEC) (Monk 2003; Arnold, Falk \& Winther 2006, 2010; Christiansen, Munthe-Kaas \& Owren 2011). We provide a semi-discretization of the noncanonical Vlasov-Maxwell Poisson structure, which preserves the defining properties of the bracket, anti-symmetry and the Jacobi identity, as well as its Casimir invariants, implying that the semi-discrete system is still a Hamiltonian system. Due to the generality of our framework, the aforementioned conservation properties are guaranteed independently of a particular choice of the finite element basis, as long as the corresponding finite element spaces satisfy certain compatibility conditions. In particular, this includes the spline spaces presented in $\S 3.4$. In order to ensure that these properties are also conserved by the fully discrete numerical scheme, the semi-discrete bracket is used in conjunction with Poisson time integrators provided by the previously mentioned splitting method (Crouseilles et al. 2015; He et al. 2015; Qin et al. 2015) and higher-order compositions thereof. A semi-discretization of the noncanonical Hamiltonian structure of the relativistic Vlasov-Maxwell system with spin and that for the gyrokinetic Vlasov-Maxwell system have recently been described by Burby (2017).

It is worth emphasizing that the aim and use of preserving the Hamiltonian structure in the course of discretization is not limited to good energy and momentum conservation properties. These are merely by-products but not the goal of the effort. Furthermore, from a practical point of view, the significance of global energy or momentum conservation by some numerical scheme for some Hamiltonian partial differential equation should not be overestimated. Of course, these are important properties of any Hamiltonian system and should be preserved within suitable error bounds in any numerical simulation. However, when performing a semi-discretization in space, the resulting finite-dimensional system of ordinary differential equations usually has millions or billions degrees of freedom. Conserving only a very small number of invariants hardly restricts the numerical solution of such a large system. It is not difficult to perceive that one can conserve the total energy of a system in a simulation and still obtain false or even unphysical results. It is much more useful to preserve local conservation laws like the local energy and momentum balance or multi-symplecticity (Reich 2000; Moore \& Reich 2003), thus posing much more severe restrictions on the numerical solution than just conserving the total energy of the system. A symplectic or Poisson integrator, on the other hand, preserves the whole hierarchy of Poincaré integral invariants of the finite-dimensional system (Channell \& Scovel 1990; Sanz-Serna \& Calvo 1993). For a Hamiltonian system of ordinary differential equations with $n$ degrees of freedom, e.g. obtained from a semi-discrete Poisson bracket, these are $n$ invariants. In addition, such integrators often preserve Noether symmetries and the associated local conservation laws as well as Casimir invariants.

We proceed as follows. In $\S 2$, we provide a short review of the Vlasov-Maxwell system and its Poisson bracket formulation, including a discussion of the Jacobi identity, Casimir invariants and invariants commuting with the specific VlasovMaxwell Hamiltonian. In $\S 3$, we introduce the finite element exterior calculus framework using the example of Maxwell's equation, we introduce the deRham complex and finite element spaces of differential forms. The actual discretization of the Poisson bracket is performed in $\S 4$. We prove the discrete Jacobi identity and the conservation of discrete Casimir invariants, including the discrete Gauss' law. In $\S 5$, we introduce a splitting for the Vlasov-Maxwell Hamiltonian, which leads to an explicit time stepping scheme. Various compositions are used in order to obtain 
higher-order methods. Backward error analysis is used in order to study the long-time energy behaviour. In $\S 6$, we apply the method to the Vlasov-Maxwell system in $1 \mathrm{~d} 2 \mathrm{v}$ (one spatial and two velocity dimensions) using splines for the discretization of the fields. Section 7 concludes the paper with numerical experiments, using nonlinear Landau damping and the Weibel instability to verify the favourable properties of our scheme.

\section{The Vlasov-Maxwell system}

The non-relativistic Vlasov equation for a particle species $s$ of charge $q_{s}$ and mass $m_{s}$ reads

$$
\frac{\partial f_{s}}{\partial t}+\boldsymbol{v} \cdot \nabla_{x} f_{s}+\frac{q_{s}}{m_{s}}(\boldsymbol{E}+\boldsymbol{v} \times \boldsymbol{B}) \cdot \nabla_{v} f_{s}=0,
$$

and couples nonlinearly to the Maxwell equations,

$$
\begin{aligned}
\frac{\partial \boldsymbol{E}}{\partial t}-\operatorname{curl} \boldsymbol{B} & =-\boldsymbol{J}, \\
\frac{\partial \boldsymbol{B}}{\partial t}+\operatorname{curl} \boldsymbol{E} & =0, \\
\operatorname{div} \boldsymbol{E} & =\rho, \\
\operatorname{div} \boldsymbol{B} & =0 .
\end{aligned}
$$

These equations are to be solved with suitable initial and boundary conditions. Here, $(\boldsymbol{x}, \boldsymbol{v})$ denotes the phasespace coordinates, $f_{s}$ is the phase space distribution function of particle species $s, \boldsymbol{E}$ is the electric field and $\boldsymbol{B}$ is the magnetic flux density (or induction), which we will refer to as the magnetic field as is prevalent in the plasma physics literature, and we have scaled the variables, but retained the mass $m_{s}$ and the signed charge $q_{s}$ to distinguish species. Observe that we use grad, curl, div to denote $\nabla_{x}, \nabla_{x} \times, \nabla_{x} \cdot$, respectively, when they act on variables depending only on $x$. The sources for the Maxwell equations, the charge density $\rho$ and the current density $\boldsymbol{J}$, are obtained from the distribution functions $f_{s}$ by

$$
\rho=\sum_{s} q_{s} \int f_{s} \mathrm{~d} \boldsymbol{v}, \quad \boldsymbol{J}=\sum_{s} q_{s} \int f_{s} \boldsymbol{v} \mathrm{d} \boldsymbol{v} .
$$

Taking the divergence of Ampère's equation (2.2) and using Gauss' law (2.4) gives the continuity equation for charge conservation

$$
\frac{\partial \rho}{\partial t}+\operatorname{div} \boldsymbol{J}=0
$$

Equation (2.7) serves as a compatibility condition for Maxwell's equations, which are ill posed when (2.7) is not satisfied. Moreover it can be shown that if the divergence constraints (2.4) and (2.5) are satisfied at the initial time, they remain satisfied for all times by the solution of Ampère's equation (2.2) and Faraday's law (2.3), which have a unique solution by themselves provided adequate initial and boundary conditions are imposed. This follows directly from the fact that the divergence of the curl vanishes and (2.7). The continuity equation follows from the Vlasov equation by integration over velocity space and using the definitions of charge and current densities. However this does not necessarily remain true when the charge and current 
densities are approximated numerically. The problem for numerical methods is then to find a way to have discrete sources, which satisfy a discrete continuity equation compatible with the discrete divergence and curl operators. Another option is to modify the Maxwell equations, so that they are well posed independently of the sources, by introducing two additional scalar unknowns that can be seen as Lagrange multipliers for the divergence constraints. These should become arbitrarily small when the continuity equation is close to being satisfied.

\subsection{Non-canonical Hamiltonian structure}

The Vlasov-Maxwell system possesses a noncanonical Hamiltonian structure. The system of equations (2.1)-(2.3) can be obtained from the following Poisson bracket, a bilinear, anti-symmetric bracket that satisfies Leibniz' rule and the Jacobi identity:

$$
\begin{aligned}
\{\mathcal{F}, \mathcal{G}\}\left[f_{s}, \boldsymbol{E}, \boldsymbol{B}\right]= & \sum_{s} \int \frac{f_{s}}{m_{s}}\left[\frac{\delta \mathcal{F}}{\delta f_{s}}, \frac{\delta \mathcal{G}}{\delta f_{s}}\right] \mathrm{d} \boldsymbol{x} \mathrm{d} \boldsymbol{v} \\
& +\sum_{s} \frac{q_{s}}{m_{s}} \int f_{s}\left(\nabla_{\boldsymbol{v}} \frac{\delta \mathcal{F}}{\delta f_{s}} \cdot \frac{\delta \mathcal{G}}{\delta \boldsymbol{E}}-\nabla_{\boldsymbol{v}} \frac{\delta \mathcal{G}}{\delta f_{s}} \cdot \frac{\delta \mathcal{F}}{\delta \boldsymbol{E}}\right) \mathrm{d} \boldsymbol{x} \mathrm{d} \boldsymbol{v} \\
& +\sum_{s} \frac{q_{s}}{m_{s}^{2}} \int f_{s} \boldsymbol{B} \cdot\left(\nabla_{\boldsymbol{v}} \frac{\delta \mathcal{F}}{\delta f_{s}} \times \nabla_{\boldsymbol{v}} \frac{\delta \mathcal{G}}{\delta f_{s}}\right) \mathrm{d} \boldsymbol{x} \mathrm{d} \boldsymbol{v} \\
& +\int\left(\operatorname{curl} \frac{\delta \mathcal{F}}{\delta \boldsymbol{E}} \cdot \frac{\delta \mathcal{G}}{\delta \boldsymbol{B}}-\operatorname{curl} \frac{\delta \mathcal{G}}{\delta \boldsymbol{E}} \cdot \frac{\delta \mathcal{F}}{\delta \boldsymbol{B}}\right) \mathrm{d} \boldsymbol{x},
\end{aligned}
$$

where $[f, g]=\nabla_{x} f \cdot \nabla_{v} g-\nabla_{x} g \cdot \nabla_{v} f$. This bracket was introduced in Morrison (1980), with a term corrected in Marsden \& Weinstein (1982) (see also Weinstein \& Morrison 1981; Morrison 1982), and its limitation to divergence-free magnetic fields first pointed out in Morrison (1982). See also Chandre et al. (2013) and Morrison (2013), where the latter contains the details of the direct proof of the Jacobi identity

$$
\{\mathcal{F},\{\mathcal{G}, \mathcal{H}\}\}+\{\mathcal{G},\{\mathcal{H}, \mathcal{F}\}\}+\{\mathcal{H},\{\mathcal{F}, \mathcal{G}\}\}=0 .
$$

The time evolution of any functional $\mathcal{F}\left[f_{s}, \boldsymbol{E}, \boldsymbol{B}\right]$ is given by

$$
\frac{\mathrm{d}}{\mathrm{d} t} \mathcal{F}\left[f_{s}, \boldsymbol{E}, \boldsymbol{B}\right]=\{\mathcal{F}, \mathcal{H}\},
$$

with the Hamiltonian $\mathcal{H}$ given as the sum of the kinetic energy of the particles and the electric and magnetic field energies,

$$
\mathcal{H}=\sum_{s} \frac{m_{s}}{2} \int|\boldsymbol{v}|^{2} f_{s}(t, \boldsymbol{x}, \boldsymbol{v}) \mathrm{d} \boldsymbol{x} \mathrm{d} \boldsymbol{v}+\frac{1}{2} \int\left(|\boldsymbol{E}(t, \boldsymbol{x})|^{2}+|\boldsymbol{B}(t, \boldsymbol{x})|^{2}\right) \mathrm{d} \boldsymbol{x} .
$$

In order to obtain the Vlasov equations, we consider the functional

$$
\delta_{x v}\left[f_{s}\right]=\int f_{s}\left(t, \boldsymbol{x}^{\prime}, \boldsymbol{v}^{\prime}\right) \delta\left(\boldsymbol{x}-\boldsymbol{x}^{\prime}\right) \delta\left(\boldsymbol{v}-\boldsymbol{v}^{\prime}\right) \mathrm{d} \boldsymbol{x}^{\prime} \mathrm{d} \boldsymbol{v}^{\prime}=f_{s}(t, \boldsymbol{x}, \boldsymbol{v}),
$$

for which the equations of motion (2.10) are computed as

$$
\frac{\partial f_{s}}{\partial t}(t, \boldsymbol{x}, \boldsymbol{v})=\int \delta\left(\boldsymbol{x}-\boldsymbol{x}^{\prime}\right) \delta\left(\boldsymbol{v}-\boldsymbol{v}^{\prime}\right)\left[\frac{1}{2}\left|\boldsymbol{v}^{\prime}\right|^{2}, f_{s}\left(t, \boldsymbol{x}^{\prime}, \boldsymbol{v}^{\prime}\right)\right] \mathrm{d} \boldsymbol{x}^{\prime} \mathrm{d} \boldsymbol{v}^{\prime}
$$




$$
\begin{aligned}
& -\frac{q_{s}}{m_{s}} \int \delta\left(\boldsymbol{x}-\boldsymbol{x}^{\prime}\right) \delta\left(\boldsymbol{v}-\boldsymbol{v}^{\prime}\right)\left(\nabla_{v} f_{s}\left(t, \boldsymbol{x}^{\prime}, \boldsymbol{v}^{\prime}\right)\right) \cdot \boldsymbol{E}\left(t, \boldsymbol{x}^{\prime}\right) \mathrm{d} \boldsymbol{x}^{\prime} \mathrm{d} \boldsymbol{v}^{\prime} \\
& -\frac{q_{s}}{m_{s}} \int \delta\left(\boldsymbol{x}-\boldsymbol{x}^{\prime}\right) \delta\left(\boldsymbol{v}-\boldsymbol{v}^{\prime}\right)\left(\nabla_{v} f_{s}\left(t, \boldsymbol{x}^{\prime}, \boldsymbol{v}^{\prime}\right)\right) \cdot\left(\boldsymbol{B}\left(t, \boldsymbol{x}^{\prime}\right) \times \boldsymbol{v}^{\prime}\right) \mathrm{d} \boldsymbol{x}^{\prime} \mathrm{d} \boldsymbol{v}^{\prime} \\
= & -\boldsymbol{v} \cdot \nabla_{x} f_{s}(t, \boldsymbol{x}, \boldsymbol{v})-\frac{q_{s}}{m_{s}}(\boldsymbol{E}(t, \boldsymbol{x})+\boldsymbol{v} \times \boldsymbol{B}(t, \boldsymbol{x})) \cdot \nabla_{v} f_{s}(t, \boldsymbol{x}, \boldsymbol{v})
\end{aligned}
$$

For the electric field, we consider

$$
\delta_{x}[\boldsymbol{E}]=\int \boldsymbol{E}\left(t, \boldsymbol{x}^{\prime}\right) \delta\left(\boldsymbol{x}-\boldsymbol{x}^{\prime}\right) \mathrm{d} \boldsymbol{x}^{\prime}=\boldsymbol{E}(t, \boldsymbol{x}),
$$

so that from (2.10) we obtain Ampère's equation,

$$
\begin{aligned}
\frac{\partial \boldsymbol{E}}{\partial t}(t, \boldsymbol{x}) & =\int\left(\operatorname{curl} \boldsymbol{B}\left(t, \boldsymbol{x}^{\prime}\right)-\sum_{s} q_{s} f_{s}\left(t, \boldsymbol{x}^{\prime}, \boldsymbol{v}^{\prime}\right) \boldsymbol{v}^{\prime}\right) \delta\left(\boldsymbol{x}-\boldsymbol{x}^{\prime}\right) \mathrm{d} \boldsymbol{x}^{\prime} \mathrm{d} \boldsymbol{v}^{\prime} \\
& =\operatorname{curl} \boldsymbol{B}(t, \boldsymbol{x})-\boldsymbol{J}(t, \boldsymbol{x}),
\end{aligned}
$$

where the current density $\boldsymbol{J}$ is given by

$$
\boldsymbol{J}(t, \boldsymbol{x})=\sum_{s} q_{s} \int f_{s}(t, \boldsymbol{x}, \boldsymbol{v}) \boldsymbol{v} \mathrm{d} \boldsymbol{v} .
$$

And for the magnetic field, we consider

$$
\delta_{x}[\boldsymbol{B}]=\int \boldsymbol{B}\left(t, \boldsymbol{x}^{\prime}\right) \delta\left(\boldsymbol{x}-\boldsymbol{x}^{\prime}\right) \mathrm{d} \boldsymbol{x}^{\prime}=\boldsymbol{B}(t, \boldsymbol{x}),
$$

and obtain the Faraday equation,

$$
\frac{\partial \boldsymbol{B}}{\partial t}(t, \boldsymbol{x})=-\int\left(\operatorname{curl} \boldsymbol{E}\left(t, \boldsymbol{x}^{\prime}\right)\right) \delta\left(\boldsymbol{x}-\boldsymbol{x}^{\prime}\right) \mathrm{d} \boldsymbol{x}=-\operatorname{curl} \boldsymbol{E}(t, \boldsymbol{x}) .
$$

Our aim is to preserve this noncanonical Hamiltonian structure and its features at the discrete level. This can be done by taking only a finite number of initial positions for the particles instead of a continuum and by taking the electromagnetic fields in finitedimensional subspaces of the original function spaces. A good candidate for such a discretization is the finite element particle-in-cell framework. In order to satisfy the continuity equation as well as the identities from vector calculus and thereby preserve Gauss' law and the divergence of the magnetic field, the finite element spaces for the different fields cannot be chosen independently. The right framework is given by FEEC.

Before describing this framework in more detail, we shortly want to discuss some conservation laws of the Vlasov-Maxwell system. In Hamiltonian systems, there are two kinds of conserved quantities, Casimir invariants and momentum maps.

\subsection{Invariants}

A family of conserved quantities are Casimir invariants (Casimirs), which originate from the degeneracy of the Poisson bracket. Casimirs are functionals $\mathcal{C}\left(f_{s}, \boldsymbol{E}, \boldsymbol{B}\right)$ which Poisson commute with every other functional $\mathcal{G}\left(f_{s}, \boldsymbol{E}, \boldsymbol{B}\right)$, i.e. $\{\mathcal{C}, \mathcal{G}\}=0$. For the 
Vlasov-Maxwell bracket, there are several such Casimirs (Morrison 1987; Morrison \& Pfirsch 1989; Chandre et al. 2013). First, the integral of any real function $h_{s}$ of each distribution function $f_{s}$ is preserved, i.e.

$$
\mathcal{C}_{s}=\int h_{s}\left(f_{s}\right) \mathrm{d} \boldsymbol{x} \mathrm{d} \boldsymbol{v}
$$

This family of Casimirs is a manifestation of Liouville's theorem and corresponds to conservation of phase space volume. Further we have two Casimirs related to Gauss' law (2.4) and the divergence-free property of the magnetic field (2.5),

$$
\begin{aligned}
& \mathcal{C}_{E}=\int h_{E}(\boldsymbol{x})(\operatorname{div} \boldsymbol{E}-\rho) \mathrm{d} \boldsymbol{x}, \\
& \mathcal{C}_{B}=\int h_{B}(\boldsymbol{x}) \operatorname{div} \boldsymbol{B} \mathrm{d} \boldsymbol{x},
\end{aligned}
$$

where $h_{E}$ and $h_{B}$ are arbitrary real functions of $\boldsymbol{x}$. The latter functional, $\mathcal{C}_{B}$, is not a true Casimir but should rather be referred to as pseudo-Casimir. It acts like a Casimir in that it Poisson commutes with any other functional, but the Jacobi identity is only satisfied when $\operatorname{div} B=0$ (see Morrison 1982, 2013).

A second family of conserved quantities are momentum maps $\Phi$, which arise from symmetries that preserve the particular Hamiltonian $\mathcal{H}$, and therefore also the equations of motion. This means that the Hamiltonian is constant along the flow of $\Phi$, i.e.

$$
\{\mathcal{H}, \Phi\}=0 .
$$

From Noether's theorem it follows that the generators $\Phi$ of the symmetry are preserved by the time evolution, i.e.

$$
\frac{\mathrm{d} \Phi}{\mathrm{d} t}=0
$$

If the symmetry condition (2.22) holds, this is obvious by the anti-symmetry of the Poisson bracket as

$$
\frac{\mathrm{d} \Phi}{\mathrm{d} t}=\{\Phi, \mathcal{H}\}=-\{\mathcal{H}, \Phi\}=0
$$

Therefore $\Phi$ is a constant of motion if and only if $\{\Phi, \mathcal{H}\}=0$.

The complete set of constants of motion, the algebra of invariants, will be discussed elsewhere. However, as an example of a momentum map we shall consider here the total momentum

$$
\mathcal{P}=\sum_{s} \int m_{s} \boldsymbol{v} f_{s} \mathrm{~d} \boldsymbol{x} \mathrm{d} \boldsymbol{v}+\int \boldsymbol{E} \times \boldsymbol{B} \mathrm{d} \boldsymbol{x} .
$$

By direct computations, assuming periodic boundary conditions, it can be shown that

$$
\frac{\mathrm{d} \mathcal{P}}{\mathrm{d} t}=\{\mathcal{P}, \mathcal{H}\}=\int \boldsymbol{E}(\rho-\operatorname{div} \boldsymbol{E}) \mathrm{d} \boldsymbol{x}=\int \boldsymbol{E} Q(\boldsymbol{x}) \mathrm{d} \boldsymbol{x}
$$

defining $Q(\boldsymbol{x}):=\rho-\operatorname{div} \boldsymbol{E}$, which is a local version of the Casimir $\mathcal{C}_{E}$. Therefore, if at $t=0$ the Casimir $Q \equiv 0$, then momentum is conserved. If at $t=0$ the Casimir $Q \not \equiv 0$, then momentum is not conserved and it changes in accordance with (2.26). For a multi-species plasma $Q \equiv 0$ is equivalent to the physical requirement that Poisson's 
equation be satisfied. If for some reason it is not exactly satisfied, then we have violation of momentum conservation.

For a single species plasma, say electrons, with a neutralizing positive background charge $\rho_{B}(\boldsymbol{x})$, say ions, Poisson's equation is

$$
\operatorname{div} \boldsymbol{E}=\rho_{B}-\rho_{e} .
$$

The Poisson bracket for this case has the local Casimir

$$
Q_{e}=\operatorname{div} \boldsymbol{E}+\rho_{e},
$$

and it does not recognize the background charge. Because the background is stationary, the total momentum is

$$
\mathcal{P}=\int m_{e} \boldsymbol{v} f_{e} \mathrm{~d} \boldsymbol{x} \mathrm{d} \boldsymbol{v}+\int \boldsymbol{E} \times \boldsymbol{B} \mathrm{d} \boldsymbol{x}
$$

and it satisfies

$$
\frac{\mathrm{d} \mathcal{P}_{e}}{\mathrm{~d} t}=\left\{\mathcal{P}_{e}, \mathcal{H}\right\}=-\int \boldsymbol{E} \rho_{B}(\boldsymbol{x}) \mathrm{d} \boldsymbol{x} .
$$

We will verify this relation in the numerical experiments of $\S 7.5$.

\section{Finite element exterior calculus}

FEEC is a mathematical framework for mixed finite element methods, which uses geometrical and topological ideas for systematically analysing the stability and convergence of finite element discretizations of partial differential equations. This proved to be a particularly difficult problem for Maxwell's equation, which we will use in the following as an example for reviewing this framework.

\subsection{Maxwell's equations}

When Maxwell's equations are used in some material medium, they are best understood by introducing two additional fields. The electromagnetic properties are then defined by the electric and magnetic fields, usually denoted by $\boldsymbol{E}$ and $\boldsymbol{B}$, the displacement field $\boldsymbol{D}$ and the magnetic intensity $\boldsymbol{H}$. For simple materials, the electric field is related to the displacement field and the magnetic field to the magnetic intensity by

$$
D=\varepsilon E, \quad B=\mu H,
$$

where $\boldsymbol{\varepsilon}$ and $\boldsymbol{\mu}$ are the permittivity and permeability tensors reflecting the material properties. In vacuum they become the scalars $\varepsilon_{0}$ and $\mu_{0}$, which are unity in our scaled variables, while for more complicated media such as plasmas they can be nonlinear operators (Morrison 2013). The Maxwell equations with the four fields read

$$
\begin{aligned}
\frac{\partial \boldsymbol{D}}{\partial t}-\operatorname{curl} \boldsymbol{H} & =-\boldsymbol{J}, \\
\frac{\partial \boldsymbol{B}}{\partial t}+\operatorname{curl} \boldsymbol{E} & =0, \\
\operatorname{div} \boldsymbol{D} & =\rho, \\
\operatorname{div} \boldsymbol{B} & =0 .
\end{aligned}
$$


The mathematical interpretation of these fields become clearer when interpreting them as differential forms: $\boldsymbol{E}$ and $\boldsymbol{H}$ are 1-forms, $\boldsymbol{D}$ and $\boldsymbol{B}$ are 2-forms. The charge density $\rho$ is a 3 -form and the current density $\boldsymbol{J}$ a 2 -form. Moreover, the electrostatic potential $\phi$ is a 0 -form and the vector potential $\boldsymbol{A}$ a 1 -form. The grad, curl, div operators represent the exterior derivative applied respectively to 0 -forms, 1 -forms and 2-forms. To be more precise, there are two kinds of differential forms, depending on the orientation. Straight differential forms have an intrinsic (or inner) orientation, whereas twisted differential forms have an outer orientation, defined by the ambient space. Faraday's equation and $\operatorname{div} \boldsymbol{B}=0$ are naturally inner oriented, whereas Ampère's equation and Gauss' law are outer oriented. This knowledge can be used to define a natural discretization for Maxwell's equations. For finite difference approximations a dual mesh is needed for the discretization of twisted forms. This can already be found in Yee's scheme (Yee 1966). In the finite element context, only one mesh is used, but dual operators are used for the twisted forms. As an implication, the charge density $\rho$ will be treated as a 0 -form and the current density $J$ as a 1 -form, instead of a (twisted) 3-form and a (twisted) 2-form, respectively. Another consequence is that Ampère's equation and Gauss' law are being treated weakly while Faraday's equation and $\operatorname{div} \boldsymbol{B}=0$ are treated strongly. A detailed description of this formalism can be found, e.g. in Bossavit's lecture notes (Bossavit 2006).

\subsection{Finite element spaces of differential forms}

The full mathematical theory for the finite element discretization of differential forms is due to Arnold et al. $(2006,2010)$ and is called finite element exterior calculus (see also Monk 2003, Christiansen et al. 2011). Most finite element spaces appearing in this theory were known before, but their connection in the context of differential forms was not made clear. The first building block of FEEC is the following commuting diagram:

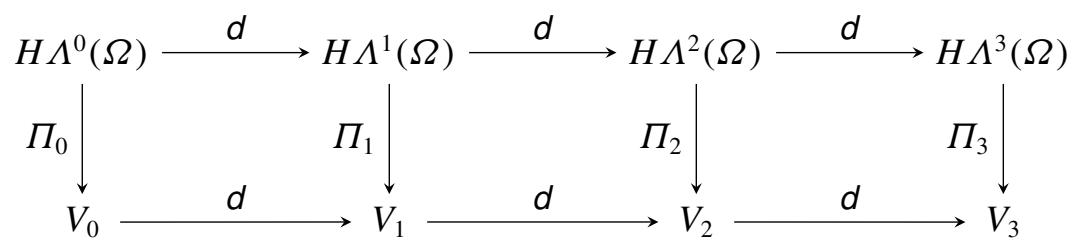

where $\Omega \subset \mathbb{R}^{3}, \Lambda^{k}(\Omega)$ is the space of $k$-forms on $\Omega$ that we endow with the inner product $\langle\alpha, \beta\rangle=\int \alpha \wedge \star \beta, \star$ is the Hodge operator and $d$ is the exterior derivative that generalizes the gradient, curl and divergence. Then we define

$$
L^{2} \Lambda^{k}(\Omega)=\left\{\omega \in \Lambda^{k}(\Omega) \mid\langle\omega, \omega\rangle<+\infty\right\}
$$

and the Sobolev spaces of differential forms

$$
H \Lambda^{k}(\Omega)=\left\{\omega \in L^{2} \Lambda^{k}(\Omega) \mid d \omega \in L^{2} \Lambda^{k+1}(\Omega)\right\} .
$$

Obviously in a three-dimensional manifold the exterior derivative of a 3-form vanishes so that $H \Lambda^{3}(\Omega)=L^{2}(\Omega)$. This diagram can also be expressed using the standard 
vector calculus formalism:

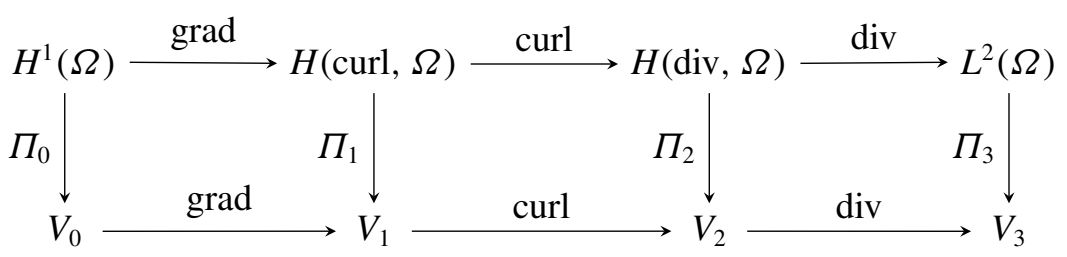

The first row of (3.9) represents the sequence of function spaces involved in Maxwell's equations. Such a sequence is called a complex if at each node, the image of the previous operator is in the kernel of the next operator, i.e. $\operatorname{Im}(\operatorname{grad}) \subseteq \operatorname{Ker}(\operatorname{curl})$ and $\operatorname{Im}(\operatorname{curl}) \subseteq \operatorname{Ker}(\operatorname{div})$. The power of the conforming finite element framework is that this complex can be reproduced at the discrete level by choosing the appropriate finitedimensional subspaces $V_{0}, V_{1}, V_{2}, V_{3}$. The order of the approximation is dictated by the choice made for $V_{0}$ and the requirement of having a complex at the discrete level. The projection operators $\Pi_{i}$ are the finite element interpolants, which have the property that the diagram is commuting. This means for example, that the grad of the projection on $V_{0}$ is identical to the projection of the grad on $V_{1}$. As proven by Arnold, Falk and Winther, their choice of finite elements naturally leads to stable discretizations.

There are many known sequences of finite element spaces that fit this diagram. The sequences proposed by Arnold, Falk and Winther are based on well-known finite element spaces. On tetrahedra these are $H^{1}$ conforming $\mathbb{P}_{k}$ Lagrange finite elements for $V_{0}$, the $H$ (curl) conforming Nédélec elements for $V_{1}$, the $H$ (div) conforming Raviart-Thomas elements for $V_{2}$ and discontinuous Galerkin elements for $V_{3}$. A similar sequence can be defined on hexahedra based on the $H^{1}$ conforming $\mathbb{Q}_{k}$ Lagrange finite elements for $V_{0}$.

Other sequences that satisfy the complex property are available. Let us in particular cite the mimetic spectral elements (Kreeft, Palha \& Gerritsma 2011; Gerritsma 2012; Palha et al. 2014) and the spline finite elements (Buffa, Sangalli \& Vázquez 2010; Buffa et al. 2011; Ratnani and Sonnendrücker 2012) that we shall use in this work, as splines are generally favoured in PIC codes due to their smoothness properties that enable noise reduction.

\subsection{Finite element discretization of Maxwell's equations}

This framework is enough to express discrete relations between all the straight (or primal forms), i.e. $\boldsymbol{E}, \boldsymbol{B}, \boldsymbol{A}$ and $\phi$. The commuting diagram yields a direct expression of the discrete Faraday equation. Indeed projecting all the components of the equation onto $V_{2}$ yields

$$
\frac{\partial \Pi_{2} \boldsymbol{B}}{\partial t}+\Pi_{2} \operatorname{curl} \boldsymbol{E}=0
$$

which is equivalent, due to the commuting diagram property, to

$$
\frac{\partial \Pi_{2} \boldsymbol{B}}{\partial t}+\operatorname{curl} \Pi_{1} \boldsymbol{E}=0 .
$$

Denoting with an $h$ index the discrete fields, $\boldsymbol{B}_{h}=\Pi_{2} \boldsymbol{B}, \boldsymbol{E}_{h}=\Pi_{1} \boldsymbol{E}$, this yields the discrete Faraday equation,

$$
\frac{\partial \boldsymbol{B}_{h}}{\partial t}+\operatorname{curl} \boldsymbol{E}_{h}=0
$$


In the same way, the discrete electric and magnetic fields are defined exactly as in the continuous case from the discrete potentials, thanks to the compatible finite element spaces,

$$
\begin{aligned}
& \boldsymbol{E}_{h}=\Pi_{1} \boldsymbol{E}=-\Pi_{1} \operatorname{grad} \phi-\Pi_{1} \frac{\partial \boldsymbol{A}}{\partial t}=-\operatorname{grad} \Pi_{0} \phi-\frac{\partial \Pi_{1} \boldsymbol{A}}{\partial t}=-\operatorname{grad} \phi_{h}-\frac{\partial \boldsymbol{A}_{h}}{\partial t}, \\
& \boldsymbol{B}_{h}=\Pi_{2} \boldsymbol{B}=\Pi_{2} \operatorname{curl} \boldsymbol{A}=\operatorname{curl} \Pi_{1} \boldsymbol{A}=\operatorname{curl} \boldsymbol{A}_{h},
\end{aligned}
$$

so that automatically we get

$$
\operatorname{div} \boldsymbol{B}_{h}=0 .
$$

On the other hand, Ampère's equation and Gauss' law relate expressions involving twisted differential forms. In the finite element framework, these should be expressed on the dual complex to (3.9). But due to the property that the dual of an operator in $L^{2}(\Omega)$ can be identified with its $L^{2}$ adjoint via an inner product, the discrete dual spaces are such that $V_{0}^{*}=V_{3}, V_{1}^{*}=V_{2}, V_{2}^{*}=V_{1}$ and $V_{3}^{*}=V_{0}$, so that the dual operators and spaces are not explicitly needed. They are most naturally used seamlessly by keeping the weak formulation of the corresponding equations. The weak form of Ampère's equation is found by taking the dot product of (2.2) with a test function $\overline{\boldsymbol{E}} \in H(\operatorname{curl}, \Omega)$ and applying a Green identity. Assuming periodic boundary conditions, the weak solution of Ampère's equation $(\boldsymbol{E}, \boldsymbol{B}) \in H(\operatorname{curl}, \Omega) \times H(\operatorname{div}, \Omega)$ is characterized by

$$
\frac{\mathrm{d}}{\mathrm{d} t} \int_{\Omega} \boldsymbol{E} \cdot \overline{\boldsymbol{E}} \mathrm{d} \boldsymbol{x}-\int_{\Omega} \boldsymbol{B} \cdot \operatorname{curl} \overline{\boldsymbol{E}} \mathrm{d} \boldsymbol{x}=-\int_{\Omega} \boldsymbol{J} \cdot \overline{\boldsymbol{E}} \mathrm{d} \boldsymbol{x} \quad \forall \overline{\boldsymbol{E}} \in H(\operatorname{curl}, \Omega) .
$$

The discrete version is obtained by replacing the continuous spaces by their finitedimensional subspaces. The approximate solution $\left(\boldsymbol{E}_{h}, \boldsymbol{B}_{h}\right) \in V_{1} \times V_{2}$ is characterized by

$$
\frac{\mathrm{d}}{\mathrm{d} t} \int_{\Omega} \boldsymbol{E}_{h} \cdot \overline{\boldsymbol{E}}_{h} \mathrm{~d} \boldsymbol{x}-\int_{\Omega} \boldsymbol{B}_{h} \cdot \operatorname{curl} \overline{\boldsymbol{E}}_{h} \mathrm{~d} \boldsymbol{x}=-\int_{\Omega} \boldsymbol{J}_{h} \cdot \overline{\boldsymbol{E}}_{h} \mathrm{~d} \boldsymbol{x} \quad \forall \overline{\boldsymbol{E}}_{h} \in V_{1} .
$$

In the same way the weak solution of Gauss' law with $\boldsymbol{E} \in H(\operatorname{curl}, \Omega)$ is characterized by

$$
\int_{\Omega} \boldsymbol{E} \cdot \operatorname{grad} \bar{\phi} \mathrm{d} \boldsymbol{x}=-\int_{\Omega} \rho \bar{\phi} \mathrm{d} \boldsymbol{x} \quad \forall \bar{\phi} \in H^{1}(\Omega),
$$

its discrete version for $\boldsymbol{E}_{h} \in V_{1}$ being characterized by

$$
\int_{\Omega} \boldsymbol{E}_{h} \cdot \operatorname{grad} \bar{\phi}_{h} \mathrm{~d} \boldsymbol{x}=-\int_{\Omega} \rho_{h} \bar{\phi}_{h} \mathrm{~d} \boldsymbol{x} \quad \forall \bar{\phi}_{h} \in V_{0} .
$$

The last step for the finite element discretization is to define a basis for each of the finite-dimensional spaces $V_{0}, V_{1}, V_{2}, V_{3}$, with $\operatorname{dim} V_{k}=N_{k}$ and to find equations relating the coefficients on these bases. Let us denote by $\left\{\Lambda_{i}^{0}\right\}_{i=1 \ldots N_{0}}$ and $\left\{\Lambda_{i}^{3}\right\}_{i=1 \ldots N_{3}}$ a basis of $V_{0}$ and $V_{3}$, respectively, which are spaces of scalar functions, and $\left\{\boldsymbol{\Lambda}_{i, \mu}^{1}\right\}_{i=1 \ldots N_{1}, \mu=1 \ldots 3}$ a basis of $V_{1} \subset H$ (curl, $\Omega$ ) and $\left\{\boldsymbol{\Lambda}_{i, \mu}^{2}\right\}_{i=1 \ldots N_{2}, \mu=1 \ldots 3}$ a basis of $V_{2} \subset H(\operatorname{div}, \Omega)$, which are vector valued functions,

$$
\boldsymbol{\Lambda}_{i, 1}^{k}=\left(\begin{array}{c}
\Lambda_{i}^{k, 1} \\
0 \\
0
\end{array}\right), \quad \boldsymbol{\Lambda}_{i, 2}^{k}=\left(\begin{array}{c}
0 \\
\Lambda_{i}^{k, 2} \\
0
\end{array}\right), \quad \boldsymbol{\Lambda}_{i, 1}^{k}=\left(\begin{array}{c}
0 \\
0 \\
\Lambda_{i}^{k, 3}
\end{array}\right), \quad k=1,2
$$


Let us note that the restriction to a basis of this form is not strictly necessary and the generalization to more general bases is straightforward. However, for didactical reasons we stick to this form of the basis as it simplifies some of the computations and thus helps to clarify the following derivations. In order to keep a concise notation, and by slight abuse of the same, we introduce vectors of basis functions $\boldsymbol{\Lambda}^{k}=\left(\boldsymbol{\Lambda}_{1,1}^{k}, \boldsymbol{\Lambda}_{1,2}^{k}, \ldots, \boldsymbol{\Lambda}_{N_{k}, 3}^{k}\right)^{\mathrm{T}}$ for $k=1,2$, which are indexed by $I=3(i-1)+\mu=$ $1 \ldots 3 N_{k}$ with $i=1 \ldots N_{k}$ and $\mu=1 \ldots 3$, and $\Lambda^{k}=\left(\Lambda_{1}^{k}, \Lambda_{2}^{k}, \ldots, \Lambda_{N_{k}}^{k}\right)^{\mathrm{T}}$ for $k=0,3$, which are indexed by $i=1 \ldots N_{k}$.

We shall also need for each basis the dual basis, which in finite element terminology corresponds to the degrees of freedom. For each basis $\Lambda_{i}^{k}$ for $k=0,3$ and $\boldsymbol{\Lambda}_{I}^{k}$ for $k=1,2$, the dual basis is denoted by $\Sigma_{i}^{k}$ and $\Sigma_{I}^{k}$, respectively, and defined by

$$
\left\langle\Sigma_{i}^{k}, \Lambda_{j}^{k}\right\rangle=\int \Sigma_{i}^{k}(\boldsymbol{x}) \Lambda_{j}^{k}(\boldsymbol{x}) \mathrm{d} \boldsymbol{x}=\delta_{i j}, \quad k=0,3,
$$

for the scalar valued bases $\Lambda_{i}^{k}$, and

$$
\left\langle\boldsymbol{\Sigma}_{I}^{k}, \boldsymbol{\Lambda}_{J}^{k}\right\rangle=\int \boldsymbol{\Sigma}_{I}^{k}(\boldsymbol{x}) \cdot \boldsymbol{\Lambda}_{J}^{k}(\boldsymbol{x}) \mathrm{d} \boldsymbol{x}=\delta_{I J}, \quad k=1,2,
$$

for the vector valued bases $\boldsymbol{\Lambda}_{I}^{k}$, where $\langle\cdot, \cdot\rangle$ denotes the $L^{2}$ inner product in the appropriate space and $\delta_{I J}$ is the Kronecker symbol, whose value is unity for $I=J$ and zero otherwise. We introduce the linear functionals $L^{2} \Lambda^{k}(\Omega) \rightarrow \mathbb{R}$, which are denoted by $\sigma_{i}^{k}$ for $k=0,3$ and by $\boldsymbol{\sigma}_{I}^{k}$ for $k=1,2$, respectively. On the finite element space they are represented by the dual basis functions $\Sigma_{i}^{k}$ and $\Sigma_{I}^{k}$ and defined by

$$
\sigma_{i}^{k}(\omega)=\left\langle\Sigma_{i}^{k}, \omega\right\rangle \quad \forall \omega \in L^{2} \Lambda^{k}(\Omega), k=0,3,
$$

and

$$
\boldsymbol{\sigma}_{I}^{k}(\boldsymbol{\omega})=\left\langle\boldsymbol{\Sigma}_{I}^{k}, \boldsymbol{\omega}\right\rangle \quad \forall \boldsymbol{\omega} \in L^{2} \Lambda^{k}(\Omega), k=1,2,
$$

so that $\sigma_{i}^{k}\left(\Lambda_{j}^{k}\right)=\delta_{i j}$ and $\boldsymbol{\sigma}_{I}^{k}\left(\boldsymbol{\Lambda}_{J}^{k}\right)=\delta_{I J}$ for the appropriate $k$. Elements of the finitedimensional spaces can be expanded on their respective bases, e.g. elements of $V_{1}$ and $V_{2}$, respectively, as

$$
\boldsymbol{E}_{h}(t, \boldsymbol{x})=\sum_{i=1}^{N_{1}} \sum_{\mu=1}^{3} e_{i, \mu}(t) \boldsymbol{\Lambda}_{i, \mu}^{1}(\boldsymbol{x}), \quad \boldsymbol{B}_{h}(t, \boldsymbol{x})=\sum_{i=1}^{N_{2}} \sum_{\mu=1}^{3} b_{i, \mu}(t) \boldsymbol{\Lambda}_{i, \mu}^{2}(\boldsymbol{x}),
$$

denoting by $\boldsymbol{e}=\left(e_{1,1}, e_{1,2}, \ldots, e_{N_{1}, 3}\right)^{\mathrm{T}} \in \mathbb{R}^{3 N_{1}}$ and $\boldsymbol{b}=\left(b_{1,1}, b_{1,2}, \ldots, b_{N_{2}, 3}\right)^{\mathrm{T}} \in \mathbb{R}^{3 N_{2}}$ the corresponding degrees of freedom with $e_{i, \mu}=\boldsymbol{\sigma}_{i, \mu}^{1}\left(\boldsymbol{E}_{h}\right)$ and $b_{i, \mu}=\boldsymbol{\sigma}_{i, \mu}^{2}\left(\boldsymbol{B}_{h}\right)$, respectively.

Denoting the elements of $\boldsymbol{e}$ by $\boldsymbol{e}_{I}$ and the elements of $\boldsymbol{b}$ by $\boldsymbol{b}_{I}$, we have that $\boldsymbol{e}_{I}=$ $\boldsymbol{\sigma}_{I}^{1}\left(\boldsymbol{E}_{h}\right)$ and $\boldsymbol{b}_{I}=\boldsymbol{\sigma}_{I}^{2}\left(\boldsymbol{B}_{h}\right)$, respectively, and can re-express (3.25) as

$$
\boldsymbol{E}_{h}(t, \boldsymbol{x})=\sum_{I=1}^{3 N_{1}} \boldsymbol{e}_{I}(t) \boldsymbol{\Lambda}_{I}^{1}(\boldsymbol{x}), \quad \boldsymbol{B}_{h}(t, \boldsymbol{x})=\sum_{I=1}^{3 N_{2}} \boldsymbol{b}_{I}(t) \boldsymbol{\Lambda}_{I}^{2}(\boldsymbol{x}) .
$$

Henceforth we will use both notations in parallel, choosing whichever is more practical at any given time. 
Due to the complex property we have that $\operatorname{curl} \boldsymbol{E}_{h} \in V_{2}$ for all $\boldsymbol{E}_{h} \in V_{1}$, so that $\operatorname{curl} \boldsymbol{E}_{h}$ can be expressed in the basis of $V_{2}$ by

$$
\operatorname{curl} \boldsymbol{E}_{h}=\sum_{i=1}^{N_{2}} \sum_{\mu=1}^{3} c_{i, \mu} \boldsymbol{\Lambda}_{i, \mu}^{2}
$$

Let us also denote by $\boldsymbol{c}=\left(c_{1,1}, c_{1,2}, \ldots, c_{N_{2}, 3}\right)^{\mathrm{T}}$, so that $\operatorname{curl} \boldsymbol{E}_{h}$ can also be written as

$$
\operatorname{curl} \boldsymbol{E}_{h}=\sum_{I=1}^{3 N_{2}} \boldsymbol{c}_{I} \boldsymbol{\Lambda}_{I}^{2} .
$$

On the other hand

$$
\left.\begin{array}{c}
\operatorname{curl} \boldsymbol{E}_{h}=\operatorname{curl}\left(\sum_{I=1}^{3 N_{1}} \boldsymbol{e}_{I} \boldsymbol{\Lambda}_{I}^{1}\right)=\sum_{I=1}^{3 N_{1}} \boldsymbol{e}_{I} \operatorname{curl} \boldsymbol{\Lambda}_{I}^{1}, \\
\boldsymbol{\sigma}_{I}^{2}\left(\operatorname{curl} \boldsymbol{E}_{h}\right)=\sum_{J=1}^{3 N_{1}} e_{J} \boldsymbol{\sigma}_{I}^{2}\left(\operatorname{curl} \boldsymbol{\Lambda}_{J}^{1}\right) .
\end{array}\right\}
$$

Denoting by $\mathbb{C}$ the discrete curl matrix,

$$
\mathbb{C}=\left(\boldsymbol{\sigma}_{I}^{2}\left(\operatorname{curl} \boldsymbol{\Lambda}_{J}^{1}\right)\right)_{1 \leqslant I \leqslant 3 N_{2}, 1 \leqslant J \leqslant 3 N_{1}},
$$

the degrees of freedom of $\operatorname{curl} \boldsymbol{E}_{h}$ in $V_{2}$ are related to the degrees of freedom of $\boldsymbol{E}_{h}$ in $V_{1}$ by $\boldsymbol{c}=\mathbb{C} \boldsymbol{e}$. In the same way we can define the discrete gradient matrix $\mathbb{G}$ and the discrete divergence matrix $\mathbb{D}$, given by

$$
\mathbb{G}=\left(\boldsymbol{\sigma}_{I}^{1}\left(\operatorname{grad} \boldsymbol{\Lambda}_{J}^{0}\right)\right)_{1 \leqslant I \leqslant 3 N_{1}, 1 \leqslant J \leqslant N_{0}} \text { and } \mathbb{D}=\left(\boldsymbol{\sigma}_{I}^{3}\left(\operatorname{div} \boldsymbol{\Lambda}_{j}^{2}\right)\right)_{1 \leqslant I \leqslant N_{3}, 1 \leqslant J \leqslant 3 N_{2}},
$$

respectively. Denoting by $\boldsymbol{\varphi}=\left(\varphi_{1}, \ldots, \varphi_{N_{0}}\right)^{\mathrm{T}}$ and $\boldsymbol{a}=\left(a_{1,1}, a_{1,2}, \ldots, a_{N_{1}, 3}\right)^{\mathrm{T}}$ the degrees of freedom of the potentials $\phi_{h}$ and $\boldsymbol{A}_{h}$, with $\varphi_{i}=\sigma_{i}^{0}\left(\phi_{h}\right)$ for $1 \leqslant i \leqslant N_{0}$ and $\boldsymbol{a}_{I}=\boldsymbol{\sigma}_{I}^{1}\left(\boldsymbol{A}_{h}\right)$ for $1 \leqslant I \leqslant 3 N_{1}$, the relation (3.13) between the discrete fields (3.25) and the potentials can be written using only the degrees of freedom as

$$
\boldsymbol{e}=-\mathbb{G} \boldsymbol{\varphi}-\frac{\mathrm{d} \boldsymbol{a}}{\mathrm{d} t}, \quad \boldsymbol{b}=\mathbb{C} \boldsymbol{a} .
$$

Finally, we need to define the so-called mass matrices in each of the discrete spaces $V_{i}$, which define the discrete Hodge operator linking the primal complex with the dual complex. We denote by $\left(\mathbb{M}_{0}\right)_{i j}=\int_{\Omega} \Lambda_{i}^{0}(\boldsymbol{x}) \Lambda_{j}^{0}(\boldsymbol{x}) \mathrm{d} \boldsymbol{x}$ with $1 \leqslant i, j \leqslant N_{0}$ and $\left(\mathbb{M}_{1}\right)_{I J}=$ $\int_{\Omega} \boldsymbol{\Lambda}_{I}^{1}(\boldsymbol{x}) \cdot \boldsymbol{\Lambda}_{J}^{1}(\boldsymbol{x}) \mathrm{d} \boldsymbol{x}$ with $1 \leqslant I, J \leqslant 3 N_{1}$ the mass matrices in $V_{0}$ and $V_{1}$, respectively, and similarly $\mathbb{M}_{2}$ and $\mathbb{M}_{3}$ the mass matrices in $V_{2}$ and $V_{3}$. Using these definitions as well as $\varrho=\left(\varrho_{1}, \ldots, \varrho_{N_{0}}\right)^{\mathrm{T}}$ and $\boldsymbol{j}=\left(j_{1,1}, j_{1,2}, \ldots, j_{N_{1}, 3}\right)^{\mathrm{T}}$ with $\varrho_{i}=\sigma_{i}^{0}\left(\rho_{h}\right)$ for $1 \leqslant i \leqslant N_{0}$ and $\boldsymbol{j}_{I}=\boldsymbol{\sigma}_{I}^{1}\left(\boldsymbol{J}_{h}\right)$ for $1 \leqslant I \leqslant 3 N_{1}$ (recall that the charge density $\rho$ is treated as a 0 -form and the current density $\boldsymbol{J}$ as a 1 -form), we obtain a system of ordinary differential equations for each of the continuous equations, namely

$$
\begin{aligned}
\mathbb{M}_{1} \frac{\mathrm{d} \boldsymbol{e}}{\mathrm{d} t}-\mathbb{C}^{\mathrm{T}} \mathbb{M}_{2} \boldsymbol{b} & =-\boldsymbol{j} \\
\frac{\mathrm{d} \boldsymbol{b}}{\mathrm{d} t}+\mathbb{C} \boldsymbol{e} & =0
\end{aligned}
$$




$$
\begin{aligned}
\mathbb{G}^{\mathrm{T}} \mathbb{M}_{1} \boldsymbol{e} & =\boldsymbol{\varrho}, \\
\mathbb{D} \boldsymbol{b} & =0 .
\end{aligned}
$$

It is worth emphasizing that $\operatorname{div} \boldsymbol{B}=0$ is satisfied in strong form, which is important for the Jacobi identity of the discretized Poisson bracket (cf. §4.4). The complex properties can also be expressed at the matrix level. The primal sequence being

$$
\mathbb{R}^{N_{0}} \stackrel{\mathbb{G}}{\longrightarrow} \mathbb{R}^{N_{1}} \stackrel{\mathbb{C}}{\longrightarrow} \mathbb{R}^{N_{2}} \stackrel{\mathbb{D}}{\longrightarrow} \mathbb{R}^{N_{3}},
$$

with $\operatorname{Im} \mathbb{G} \subseteq \operatorname{Ker} \mathbb{C}, \operatorname{Im} \mathbb{C} \subseteq \operatorname{Ker} \mathbb{D}$, and the dual sequence being

$$
\mathbb{R}^{N_{3}} \stackrel{\mathbb{D}^{\mathrm{T}}}{\longrightarrow} \mathbb{R}^{N_{2}} \stackrel{\mathbb{C}^{\mathrm{T}}}{\longrightarrow} \mathbb{R}^{N_{1}} \stackrel{\mathbb{G}^{\mathrm{T}}}{\longrightarrow} \mathbb{R}^{N_{0}},
$$

with $\operatorname{Im} \mathbb{D}^{\mathrm{T}} \subseteq \operatorname{Ker} \mathbb{C}^{\mathrm{T}}, \operatorname{Im} \mathbb{C}^{\mathrm{T}} \subseteq \operatorname{Ker} \mathbb{G}^{\mathrm{T}}$

\subsection{Example: B-spline finite elements}

In the following, we will use so-called basic splines, or B-splines, as bases for the finite element function spaces. B-splines are piecewise polynomials. The points where two polynomials connect are called knots. The $j$ th basic spline (B-spline) of degree $p$ can be defined recursively by

$$
N_{j}^{p}(x)=w_{j}^{p}(x) N_{j}^{p-1}(x)+\left(1-w_{j+1}^{p}(x)\right) N_{j+1}^{p-1}(x),
$$

where

$$
w_{j}^{p}(x)=\frac{x-x_{j}}{x_{j+p}-x_{j}}
$$

and

$$
N_{j}^{0}(x)= \begin{cases}1 & x \in\left[x_{j}, x_{j+1}\right), \\ 0 & \text { else, }\end{cases}
$$

with the knot vector $\Xi=\left\{x_{i}\right\}_{1 \leqslant i \leqslant N+k}$ being a non-decreasing sequence of points. The knot vector can also contain repeated knots. If a knot $x_{i}$ has multiplicity $m$, then the $\mathrm{B}$-spline is $C^{p-m}$ continuous at $x_{i}$. The derivative of a B-spline of degree $p$ can easily be computed as the difference of two B-splines of degree $p-1$,

$$
\frac{\mathrm{d}}{\mathrm{d} x} N_{j}^{p}(x)=p\left(\frac{N_{j}^{p-1}(x)}{x_{j+p}-x_{j}}-\frac{N_{j+1}^{p-1}(x)}{x_{j+p+1}-x_{j+1}}\right) .
$$

For convenience, we introduce the following shorthand notation for differentials,

$$
D_{j}^{p}(x)=p \frac{N_{j}^{p-1}(x)}{x_{j+p}-x_{j}}
$$

In the case of an equidistant grid with grid step size $\Delta x=x_{j+1}-x_{j}$, this simplifies to

$$
D_{j}^{p}(x)=\frac{N_{j}^{p-1}(x)}{\Delta x} .
$$


Using $D_{j}^{p}$ the recursion formula (3.39) becomes

$$
\frac{\mathrm{d}}{\mathrm{d} x} N_{j}^{p}(x)=D_{j}^{p}(x)-D_{j+1}^{p}(x)
$$

In more than one dimension, we can define tensor-product B-spline basis functions, e.g. for three dimensions as

$$
N_{i j k}^{p}=N_{i}^{p}\left(x_{1}\right) \otimes N_{j}^{p}\left(x_{2}\right) \otimes N_{k}^{p}\left(x_{3}\right) .
$$

The bases of the differential form spaces will be tensor products of the basis functions $N_{i}^{p}$ and the differentials $D_{j}^{p}$. The discrete function spaces are given by

$$
\begin{aligned}
& \Lambda_{h}^{0}(\Omega)=\operatorname{span}\left\{N_{i}^{p}\left(x_{1}\right) N_{j}^{p}\left(x_{2}\right) N_{k}^{p}\left(x_{3}\right) \mid 1 \leqslant i \leqslant N_{1}, 1 \leqslant j \leqslant N_{2}, 1 \leqslant k \leqslant N_{3}\right\}, \\
& \Lambda_{h}^{1}(\Omega)=\operatorname{span}\left\{\left(\begin{array}{c}
D_{i}^{p}\left(x_{1}\right) N_{j}^{p}\left(x_{2}\right) N_{k}^{p}\left(x_{3}\right) \\
0 \\
0
\end{array}\right),\left(\begin{array}{c}
0 \\
N_{i}^{p}\left(x_{1}\right) D_{j}^{p}\left(x_{2}\right) N_{k}^{p}\left(x_{3}\right) \\
0
\end{array}\right)\right. \text {, } \\
& \left.\times\left(\begin{array}{c}
0 \\
0 \\
N_{i}^{p}\left(x_{1}\right) N_{j}^{p}\left(x_{2}\right) D_{k}^{p}\left(x_{3}\right)
\end{array}\right) \mid 1 \leqslant i \leqslant N_{1}, 1 \leqslant j \leqslant N_{2}, 1 \leqslant k \leqslant N_{3}\right\}, \\
& \Lambda_{h}^{2}(\Omega)=\operatorname{span}\left\{\left(\begin{array}{c}
N_{i}^{p}\left(x_{1}\right) D_{j}^{p}\left(x_{2}\right) D_{k}^{p}\left(x_{3}\right) \\
0 \\
0
\end{array}\right),\left(\begin{array}{c}
0 \\
D_{i}^{p}\left(x_{1}\right) N_{j}^{p}\left(x_{2}\right) D_{k}^{p}\left(x_{3}\right) \\
0
\end{array}\right)\right. \text {, } \\
& \left.\times\left(\begin{array}{c}
0 \\
0 \\
D_{i}^{p}\left(x_{1}\right) D_{j}^{p}\left(x_{2}\right) N_{k}^{p}\left(x_{3}\right)
\end{array}\right) \mid 1 \leqslant i \leqslant N_{1}, 1 \leqslant j \leqslant N_{2}, 1 \leqslant k \leqslant N_{3}\right\}, \\
& \Lambda_{h}^{3}(\Omega)=\operatorname{span}\left\{D_{i}^{p}\left(x_{1}\right) D_{j}^{p}\left(x_{2}\right) D_{k}^{p}\left(x_{3}\right) \mid 1 \leqslant i \leqslant N_{1}, 1 \leqslant j \leqslant N_{2}, 1 \leqslant k \leqslant N_{3}\right\} .
\end{aligned}
$$

These choices appear quite natural when one considers the action of the gradient on 0 -forms, the curl on 1-forms and the divergence on 2-forms. In the following, we will exemplify this using the potentials and fields of Maxwell's equations. The semidiscrete potentials are written in the respective spline basis (3.47) as

$$
\phi_{h}(t, \boldsymbol{x})=\sum_{i, j, k} \varphi_{i j k}(t) N_{i}^{p}\left(x_{1}\right) N_{j}^{p}\left(x_{2}\right) N_{k}^{p}\left(x_{3}\right),
$$

and

$$
\boldsymbol{A}_{h}(t, \boldsymbol{x})=\sum_{i, j, k}\left(\begin{array}{l}
a_{i j k}^{1}(t) D_{i}^{p}\left(x_{1}\right) N_{j}^{p}\left(x_{2}\right) N_{k}^{p}\left(x_{3}\right) \\
a_{i j k}^{2}(t) N_{i}^{p}\left(x_{1}\right) D_{j}^{p}\left(x_{2}\right) N_{k}^{p}\left(x_{3}\right) \\
a_{i j k}^{3}(t) N_{i}^{p}\left(x_{1}\right) N_{j}^{p}\left(x_{2}\right) D_{k}^{p}\left(x_{3}\right)
\end{array}\right) .
$$

Computing the gradient of the semi-discrete scalar potential $\phi_{h}$, we find

$$
\begin{aligned}
\operatorname{grad} \phi_{h} & =\sum_{i, j, k} \varphi_{i j k}(t) \operatorname{grad}\left[N_{i}^{p}\left(x_{1}\right) N_{j}^{p}\left(x_{2}\right) N_{k}^{p}\left(x_{3}\right)\right], \\
& =\sum_{i, j, k} \varphi_{i j k}(t)\left(\begin{array}{l}
{\left[D_{i}^{p}\left(x_{1}\right)-D_{i+1}^{p}\left(x_{1}\right)\right] N_{j}^{p}\left(x_{2}\right) N_{k}^{p}\left(x_{3}\right)} \\
N_{i}^{p}\left(x_{1}\right)\left[D_{j}^{p}\left(x_{2}\right)-D_{j+1}^{p}\left(x_{2}\right)\right] N_{k}^{p}\left(x_{3}\right) \\
N_{i}^{p}\left(x_{1}\right) N_{j}^{p}\left(x_{2}\right)\left[D_{k}^{p}\left(x_{3}\right)-D_{k+1}^{p}\left(x_{3}\right)\right]
\end{array}\right) .
\end{aligned}
$$


Assuming periodic boundary conditions, the sums can be re-arranged to give

$$
\operatorname{grad} \phi_{h}=\sum_{i, j, k}\left(\begin{array}{l}
{\left[\varphi_{i j k}(t)-\varphi_{i-1 j k}(t)\right] D_{i}^{p}\left(x_{1}\right) N_{j}^{p}\left(x_{2}\right) N_{k}^{p}\left(x_{3}\right)} \\
{\left[\varphi_{i j k}(t)-\varphi_{i j-1 k}(t)\right] N_{i}^{p}\left(x_{1}\right) D_{j}^{p}\left(x_{2}\right) N_{k}^{p}\left(x_{3}\right)} \\
{\left[\varphi_{i j k}(t)-\varphi_{i j k-1}(t)\right] N_{i}^{p}\left(x_{1}\right) N_{j}^{p}\left(x_{2}\right) D_{k}^{p}\left(x_{3}\right)}
\end{array}\right)
$$

Similarly the curl of the semi-discrete vector potential $\boldsymbol{A}_{h}$ is computed as

$$
\begin{aligned}
\operatorname{curl} \boldsymbol{A}_{h}= & \sum_{i, j, k}\left(\begin{array}{c}
a_{i j k}^{3}(t) N_{i}^{p}\left(x_{1}\right)\left[D_{j}^{p}\left(x_{2}\right)-D_{j+1}^{p}\left(x_{2}\right)\right] D_{k}^{p}\left(x_{3}\right) \\
a_{i j k}^{1}(t) D_{i}^{p}\left(x_{1}\right) N_{j}^{p}\left(x_{2}\right)\left[D_{k}^{p}\left(x_{3}\right)-D_{k+1}^{p}\left(x_{3}\right)\right] \\
a_{i j k}^{2}(t)\left[D_{i}^{p}\left(x_{1}\right)-D_{i+1}^{p}\left(x_{1}\right)\right] D_{j}^{p}\left(x_{2}\right) N_{k}^{p}\left(x_{3}\right)
\end{array}\right) \\
& -\sum_{i, j, k}\left(\begin{array}{c}
a_{i j k}^{2}(t) N_{i}^{p}\left(x_{1}\right) D_{j}^{p}\left(x_{2}\right)\left[D_{k}^{p}\left(x_{3}\right)-D_{k+1}^{p}\left(x_{3}\right)\right] \\
a_{i j k}^{3}(t)\left[D_{i}^{p}\left(x_{1}\right)-D_{i+1}^{p}\left(x_{1}\right)\right] N_{j}^{p}\left(x_{2}\right) D_{k}^{p}\left(x_{3}\right) \\
a_{i j k}^{1}(t) D_{i}^{p}\left(x_{1}\right)\left[D_{j}^{p}\left(x_{2}\right)-D_{j+1}^{p}\left(x_{2}\right)\right] N_{k}^{p}\left(x_{3}\right)
\end{array}\right) .
\end{aligned}
$$

Again, assuming periodic boundary conditions, the sums can be re-arranged to give

$$
\operatorname{curl} \boldsymbol{A}_{h}=\sum_{i, j, k}\left(\begin{array}{l}
\left(\left[a_{i j k}^{3}(t)-a_{i j-1 k}^{3}(t)\right]-\left[a_{i j k}^{2}(t)-a_{i j k-1}^{2}(t)\right]\right) N_{i}^{p}\left(x_{1}\right) D_{j}^{p}\left(x_{2}\right) D_{k}^{p}\left(x_{3}\right) \\
\left(\left[a_{i j k}^{1}(t)-a_{i j k-1}^{1}(t)\right]-\left[a_{i j k}^{3}(t)-a_{i-1 j k}^{3}(t)\right]\right) D_{i}^{p}\left(x_{1}\right) N_{j}^{p}\left(x_{2}\right) D_{k}^{p}\left(x_{3}\right) \\
\left(\left[a_{i j k}^{2}(t)-a_{i-1 j k}^{2}(t)\right]-\left[a_{i j k}^{1}(t)-a_{i j-1 k}^{1}(t)\right]\right) D_{i}^{p}\left(x_{1}\right) D_{j}^{p}\left(x_{2}\right) N_{k}^{p}\left(x_{3}\right)
\end{array}\right) .
$$

Given the above, we determine the semi-discrete electromagnetic fields $\boldsymbol{E}_{h}$ and $\boldsymbol{B}_{h}$. The electric field $\boldsymbol{E}_{h}=-\operatorname{grad} \phi_{h}-\partial \boldsymbol{A}_{h} / \partial t$ is computed as

$$
\boldsymbol{E}_{h}(t, \boldsymbol{x})=\sum_{i, j, k}\left(\begin{array}{l}
{\left[\varphi_{i-1 j k}(t)-\varphi_{i j k}(t)-\dot{a}_{i j k}^{1}(t)\right] D_{i}^{p}\left(x_{1}\right) N_{j}^{p}\left(x_{2}\right) N_{k}^{p}\left(x_{3}\right)} \\
{\left[\varphi_{i j-1 k}(t)-\varphi_{i j k}(t)-\dot{a}_{i j k}^{2}(t)\right] N_{i}^{p}\left(x_{1}\right) D_{j}^{p}\left(x_{2}\right) N_{k}^{p}\left(x_{3}\right)} \\
{\left[\varphi_{i j k-1}(t)-\varphi_{i j k}(t)-\dot{a}_{i j k}^{3}(t)\right] N_{i}^{p}\left(x_{1}\right) N_{j}^{p}\left(x_{2}\right) D_{k}^{p}\left(x_{3}\right)}
\end{array}\right),
$$

and the magnetic field $\boldsymbol{B}_{h}=\operatorname{curl} \boldsymbol{A}_{h}$ as

$$
\boldsymbol{B}_{h}(t, \boldsymbol{x})=\sum_{i, j, k}\left(\begin{array}{l}
{\left[\left(a_{i j k}^{3}(t)-a_{i j-1 k}^{3}(t)\right)-\left(a_{i j k}^{2}(t)-a_{i j k-1}^{2}(t)\right)\right] N_{i}^{p}\left(x_{1}\right) D_{j}^{p}\left(x_{2}\right) D_{k}^{p}\left(x_{3}\right)} \\
{\left[\left(a_{i j k}^{1}(t)-a_{i j k-1}^{1}(t)\right)-\left(a_{i j k}^{3}(t)-a_{i-1 j k}^{3}(t)\right)\right] D_{i}^{p}\left(x_{1}\right) N_{j}^{p}\left(x_{2}\right) D_{k}^{p}\left(x_{3}\right)} \\
{\left[\left(a_{i j k}^{2}(t)-a_{i-1 j k}^{2}(t)\right)-\left(a_{i j k}^{1}(t)-a_{i j-1 k}^{1}(t)\right)\right] D_{i}^{p}\left(x_{1}\right) D_{j}^{p}\left(x_{2}\right) N_{k}^{p}\left(x_{3}\right)}
\end{array}\right) .
$$

Now it becomes clear why definitions (3.47) are the natural choice for the spline bases and it is straightforward to verify that $\operatorname{div} \boldsymbol{B}_{h}=0$.

\section{Discretization of the Hamiltonian structure}

The continuous bracket (2.8) is for the Eulerian (as opposed to Lagrangian) formulation of the Vlasov equation, and operates on functionals of the distribution function and the electric and magnetic fields. We incorporate a discretization that uses a finite number of characteristics instead of the continuum particle distribution function. This is done by localizing the distribution function on particles, which amounts to a Monte Carlo discretization of the first three integrals in (2.8) if the initial phase space positions are randomly drawn. Moreover instead of allowing the fields $\boldsymbol{E}$ and $\boldsymbol{B}$ to vary in $H(\operatorname{curl}, \Omega)$ and $H(\operatorname{div}, \Omega)$, respectively, we keep them in 
the discrete subspaces $V_{1}$ and $V_{2}$. This procedure yields a discrete Poisson bracket, from which one obtains the dynamics of a large but finite number of degrees of freedom: the particle phase space positions $\boldsymbol{z}_{a}=\left(\boldsymbol{x}_{a}, \boldsymbol{v}_{a}\right)$, where $a=1, \ldots, N_{p}$, with $N_{p}$ the number of particles, and the coefficients of the fields in the finite element basis, where we denote by $\boldsymbol{e}_{I}$ the degrees of freedom for $\boldsymbol{E}_{h}$ and by $\boldsymbol{b}_{I}$ the degrees of freedom for $\boldsymbol{B}_{h}$, as introduced in $\S 3$. The FEEC framework of $\S 3$ automatically provides the following discretization spaces for the potentials, the fields and the densities:

$$
\phi_{h}, \rho_{h} \in V_{0}, \quad \boldsymbol{A}_{h}, \boldsymbol{E}_{h}, \boldsymbol{J}_{h} \in V_{1}, \quad \boldsymbol{B}_{h} \in V_{2} .
$$

Recall that the coefficient vectors of the fields are denoted $\boldsymbol{e}$ and $\boldsymbol{b}$. In order to also get a vector expression for the particle quantities, we denote by

$$
\boldsymbol{X}=\left(\boldsymbol{x}_{1}, \ldots, \boldsymbol{x}_{N_{p}}\right)^{\mathrm{T}}, \quad \boldsymbol{V}=\left(\boldsymbol{v}_{1}, \ldots, \boldsymbol{v}_{N_{p}}\right)^{\mathrm{T}} .
$$

We use this setting to transform (2.8) into a discrete Poisson bracket for the dynamics of the coefficients $\boldsymbol{e}, \boldsymbol{b}, \boldsymbol{X}$ and $\boldsymbol{V}$.

\subsection{Discretization of the functional field derivatives}

Upon inserting (3.25), any functional $\mathcal{F}\left[\boldsymbol{E}_{h}\right]$ can be considered as a function $F(\boldsymbol{e})$ of the finite element coefficients,

$$
\mathcal{F}\left[\boldsymbol{E}_{h}\right]=F(\boldsymbol{e}) .
$$

Therefore, we can write the first variation of $\mathcal{F}[\boldsymbol{E}]$,

$$
\delta \mathcal{F}[\boldsymbol{E}]=\left\langle\frac{\delta \mathcal{F}[\boldsymbol{E}]}{\delta \boldsymbol{E}}, \delta \boldsymbol{E}\right\rangle,
$$

as

$$
\left\langle\frac{\delta \mathcal{F}\left[\boldsymbol{E}_{h}\right]}{\delta \boldsymbol{E}}, \overline{\boldsymbol{E}}_{h}\right\rangle_{L^{2}}=\left\langle\frac{\partial F(\boldsymbol{e})}{\partial \boldsymbol{e}}, \overline{\boldsymbol{e}}\right\rangle_{\mathbb{R}^{N_{1}}},
$$

with

$$
\overline{\boldsymbol{E}}_{h}(t, \boldsymbol{x})=\sum_{I=1}^{3 N_{1}} \overline{\boldsymbol{e}}_{I}(t) \boldsymbol{\Lambda}_{I}^{1}(\boldsymbol{x}), \quad \overline{\boldsymbol{e}}=\left(\bar{e}_{1,1}, \bar{e}_{1,2}, \ldots, \bar{e}_{N_{1}, 3}\right)^{\mathrm{T}} \in \mathbb{R}^{3 N_{1}}
$$

Let $\boldsymbol{\Sigma}_{I}^{1}(\boldsymbol{x})$ denote the dual basis of $\boldsymbol{\Lambda}_{I}^{1}(\boldsymbol{x})$ with respect to the $L^{2}$ inner product (3.24) and let us express the functional derivative on this dual basis, i.e.

$$
\frac{\delta \mathcal{F}\left[\boldsymbol{E}_{h}\right]}{\delta \boldsymbol{E}}=\sum_{I=1}^{3 N_{1}} \boldsymbol{f}_{I}(t) \boldsymbol{\Sigma}_{I}^{1}(\boldsymbol{x}), \quad \boldsymbol{f}=\left(f_{1,1}, f_{1,2}, \ldots, f_{N_{1}, 3}\right)^{\mathrm{T}} \in \mathbb{R}^{3 N_{1}}
$$

Using (4.5) for $\overline{\boldsymbol{e}}=(0, \ldots, 0,1,0,, 0)^{\mathrm{T}}$ with 1 at the $I$ th position and 0 everywhere else, so that $\overline{\boldsymbol{E}}_{h}=\boldsymbol{\Lambda}_{I}^{1}$, we find that

$$
\boldsymbol{f}_{I}=\frac{\partial F(\boldsymbol{e})}{\partial \boldsymbol{e}_{I}}
$$

and can therefore write

$$
\frac{\delta \mathcal{F}\left[\boldsymbol{E}_{h}\right]}{\delta \boldsymbol{E}}=\sum_{I=1}^{3 N_{1}} \frac{\partial F(\boldsymbol{e})}{\partial \boldsymbol{e}_{I}} \boldsymbol{\Sigma}_{I}^{1}(\boldsymbol{x})
$$


On the other hand, expanding the dual basis in the original basis,

$$
\boldsymbol{\Sigma}_{I}^{1}(\boldsymbol{x})=\sum_{J=1}^{3 N_{1}} \boldsymbol{a}_{I J} \boldsymbol{\Lambda}_{J}^{1}(\boldsymbol{x})
$$

and taking the $L^{2}$ inner product with $\boldsymbol{\Lambda}_{J}^{1}(\boldsymbol{x})$, we find that the matrix $\mathbb{A}=\left(\boldsymbol{a}_{I J}\right)$ verifies $\mathbb{A M}_{1}=\mathbb{I}_{3 N_{1}}$, where $\mathbb{I}_{3 N_{1}}$ denotes the $3 N_{1} \times 3 N_{1}$ identity matrix, so that $\mathbb{A}$ is the inverse of the mass matrix $\mathbb{M}_{1}$ and

$$
\frac{\delta \mathcal{F}\left[\boldsymbol{E}_{h}\right]}{\delta \boldsymbol{E}}=\sum_{I, J=1}^{3 N_{1}} \frac{\partial F(\boldsymbol{e})}{\partial \boldsymbol{e}_{I}}\left(\mathbb{M}_{1}^{-1}\right)_{I J} \boldsymbol{\Lambda}_{J}^{1}(\boldsymbol{x}) .
$$

In full analogy we find

$$
\frac{\delta \mathcal{F}\left[\boldsymbol{B}_{h}\right]}{\delta \boldsymbol{B}}=\sum_{I, J=1}^{3 N_{2}} \frac{\partial F(\boldsymbol{b})}{\partial \boldsymbol{b}_{I}}\left(\mathbb{M}_{2}^{-1}\right)_{I J} \boldsymbol{\Lambda}_{J}^{2}(\boldsymbol{x}) .
$$

Next, using (3.30), we find

$$
\operatorname{curl} \frac{\delta \mathcal{F}\left[\boldsymbol{E}_{h}\right]}{\delta \boldsymbol{E}}=\sum_{I, J=1}^{3 N_{1}} \sum_{K=1}^{3 N_{2}} \frac{\partial F(\boldsymbol{e})}{\partial \boldsymbol{e}_{I}}\left(\mathbb{M}_{1}^{-1}\right)_{I J} \mathbb{C}_{J K} \boldsymbol{\Lambda}_{K}^{2}(\boldsymbol{x}) .
$$

Finally, we can re-express the following term in the Poisson bracket

$$
\begin{aligned}
& \int \operatorname{curl} \frac{\delta \mathcal{F}\left[\boldsymbol{E}_{h}\right]}{\delta \boldsymbol{E}} \cdot \frac{\delta \mathcal{G}\left[\boldsymbol{B}_{h}\right]}{\delta \boldsymbol{B}} \mathrm{d} \boldsymbol{x} \\
& =\sum_{I, J=1}^{3 N_{1}} \sum_{K, L, M=1}^{3 N_{2}} \frac{\partial F(\boldsymbol{e})}{\partial \boldsymbol{e}_{I}}\left(\mathbb{M}_{1}^{-1}\right)_{I J} \mathbb{C}_{J K} \frac{\partial G(\boldsymbol{b})}{\partial \boldsymbol{b}_{L}}\left(\mathbb{M}_{2}^{-1}\right)_{L M} \int \boldsymbol{\Lambda}_{K}^{2}(\boldsymbol{x}) \cdot \boldsymbol{\Lambda}_{M}^{2}(\boldsymbol{x}) \mathrm{d} \boldsymbol{x} \\
& =\sum_{I, J=1}^{3 N_{1}} \sum_{K=1}^{3 N_{2}} \frac{\partial F(\boldsymbol{e})}{\partial \boldsymbol{e}_{I}}\left(\mathbb{M}_{1}^{-1}\right)_{I J} \mathbb{C}_{J K} \frac{\partial G(\boldsymbol{b})}{\partial \boldsymbol{b}_{K}}=\sum_{I=1}^{3 N_{1}} \sum_{K=1}^{3 N_{2}} \frac{\partial F(\boldsymbol{e})}{\partial \boldsymbol{e}_{I}}\left(\mathbb{M}_{1}^{-1} \mathbb{C}\right)_{I K} \frac{\partial G(\boldsymbol{b})}{\partial \boldsymbol{b}_{K}} .
\end{aligned}
$$

The other terms in the bracket involving functional derivatives with respect to the fields are handled similarly. In the next step we need to discretize the distribution function and the corresponding functional derivatives.

\subsection{Discretization of the functional particle derivatives}

We proceed by assuming a particle-like distribution function for $N_{p}$ particles labelled by $a$,

$$
f_{h}(\boldsymbol{x}, \boldsymbol{v}, t)=\sum_{a=1}^{N_{p}} w_{a} \delta\left(\boldsymbol{x}-\boldsymbol{x}_{a}(t)\right) \delta\left(\boldsymbol{v}-\boldsymbol{v}_{a}(t)\right),
$$

with mass $m_{a}$, charge $q_{a}$, weights $w_{a}$, particle positions $\boldsymbol{x}_{a}$ and particle velocities $\boldsymbol{v}_{a}$. Here, $N_{p}$ denotes the total number of particles of all species, with each particle carrying its own mass and signed charge. Functionals of the distribution function, 
$\mathcal{F}[f]$, can be considered as functions of the particle phase space trajectories, $F(\boldsymbol{X}, \boldsymbol{V})$, upon inserting (4.15),

$$
\mathcal{F}\left[f_{h}\right]=F(\boldsymbol{X}, \boldsymbol{V}) .
$$

Variation of the left-hand side and integration by parts gives,

$$
\begin{aligned}
\delta \mathcal{F}\left[f_{h}\right]= & \int \frac{\delta \mathcal{F}}{\delta f} \delta f_{h} \mathrm{~d} \boldsymbol{x} \mathrm{d} \boldsymbol{v} \\
= & -\sum_{a=1}^{N_{p}} w_{a} \int \frac{\delta \mathcal{F}}{\delta f}\left(\delta\left(\boldsymbol{v}-\boldsymbol{v}_{a}(t)\right) \nabla_{x} \delta\left(\boldsymbol{x}-\boldsymbol{x}_{a}(t)\right) \cdot \delta \boldsymbol{x}_{a}\right. \\
& \left.+\delta\left(\boldsymbol{x}-\boldsymbol{x}_{a}(t)\right) \nabla_{\boldsymbol{v}} \delta\left(\boldsymbol{v}-\boldsymbol{v}_{a}(t)\right) \cdot \delta \boldsymbol{v}_{a}\right) \mathrm{d} \boldsymbol{x} \mathrm{d} \boldsymbol{v} \\
= & \sum_{a=1}^{N_{p}} w_{a}\left(\left.\nabla_{x} \frac{\delta \mathcal{F}}{\delta f}\right|_{\left(x_{a}, \boldsymbol{v}_{a}\right)} \cdot \delta \boldsymbol{x}_{a}+\left.\nabla_{\boldsymbol{v}} \frac{\delta \mathcal{F}}{\delta f}\right|_{\left(\boldsymbol{x}_{a}, \boldsymbol{v}_{a}\right)} \cdot \delta \boldsymbol{v}_{a}\right) .
\end{aligned}
$$

Upon equating this expression with the variation of the right-hand side of (4.16),

$$
\delta F(\boldsymbol{X}, \boldsymbol{V})=\sum_{a=1}^{N_{p}}\left(\frac{\partial F}{\partial \boldsymbol{x}_{a}} \delta \boldsymbol{x}_{a}+\frac{\partial F}{\partial \boldsymbol{v}_{a}} \delta \boldsymbol{v}_{a}\right),
$$

we obtain

$$
\frac{\partial F}{\partial \boldsymbol{x}_{a}}=\left.w_{a} \nabla_{x} \frac{\delta \mathcal{F}}{\delta f}\right|_{\left(\boldsymbol{x}_{a}, \boldsymbol{v}_{a}\right)} \quad \text { and } \quad \frac{\partial F}{\partial \boldsymbol{v}_{a}}=\left.w_{a} \nabla_{\boldsymbol{v}} \frac{\delta \mathcal{F}}{\delta f}\right|_{\left(\boldsymbol{x}_{a}, \boldsymbol{v}_{a}\right)} .
$$

Considering the kinetic part of the Poisson bracket (2.8), the discretization proceeds in two steps. First, replace $f_{s}$ with $f_{h}$ to get

$$
\begin{aligned}
& \sum_{a=1}^{N_{p}} \int \frac{w_{a}}{m_{a}} \delta\left(\boldsymbol{x}-\boldsymbol{x}_{a}(t)\right) \delta\left(\boldsymbol{v}-\boldsymbol{v}_{a}(t)\right)\left[\frac{\delta \mathcal{F}}{\delta f}, \frac{\delta \mathcal{G}}{\delta f}\right] \mathrm{d} \boldsymbol{x} \mathrm{d} \boldsymbol{v} \\
& =\left.\sum_{a=1}^{N_{p}} \frac{w_{a}}{m_{a}}\left(\boldsymbol{\nabla}_{\boldsymbol{x}} \frac{\delta \mathcal{F}}{\delta f} \cdot \nabla_{\boldsymbol{v}} \frac{\delta \mathcal{G}}{\delta f}-\nabla_{\boldsymbol{v}} \frac{\delta \mathcal{F}}{\delta f} \cdot \nabla_{\boldsymbol{x}} \frac{\delta \mathcal{G}}{\delta f}\right)\right|_{\left(\boldsymbol{x}_{a}, \boldsymbol{v}_{a}\right)} .
\end{aligned}
$$

Then insert (4.19) in order to obtain the discrete kinetic bracket.

\subsection{Discrete Poisson bracket}

Replacing all functional derivatives in (2.8) as outlined in the previous two sections, we obtain the semi-discrete Poisson bracket

$$
\begin{aligned}
& \{F, G\}[\boldsymbol{X}, \boldsymbol{V}, \boldsymbol{e}, \boldsymbol{b}]=\sum_{a=1}^{N_{p}} \frac{1}{m_{a} w_{a}}\left(\frac{\partial F}{\partial \boldsymbol{x}_{a}} \cdot \frac{\partial G}{\partial \boldsymbol{v}_{a}}-\frac{\partial G}{\partial \boldsymbol{x}_{a}} \cdot \frac{\partial F}{\partial \boldsymbol{v}_{a}}\right) \\
& +\sum_{a=1}^{N_{p}} \sum_{I, J=1}^{3 N_{1}} \frac{q_{a}}{m_{a}}\left(\frac{\partial G}{\partial \boldsymbol{e}_{I}}\left(\mathbb{M}_{1}^{-1}\right)_{I J} \boldsymbol{\Lambda}_{J}^{1}\left(\boldsymbol{x}_{a}\right) \cdot \frac{\partial F}{\partial \boldsymbol{v}_{a}}-\frac{\partial F}{\partial \boldsymbol{e}_{I}}\left(\mathbb{M}_{1}^{-1}\right)_{I J} \boldsymbol{\Lambda}_{J}^{1}\left(\boldsymbol{x}_{a}\right) \cdot \frac{\partial G}{\partial \boldsymbol{v}_{a}}\right)
\end{aligned}
$$




$$
\begin{aligned}
& +\sum_{a=1}^{N_{p}} \sum_{I=1}^{3 N_{2}} \frac{q_{a}}{m_{a}^{2} w_{a}} \boldsymbol{b}_{I}(t) \boldsymbol{\Lambda}_{I}^{2}\left(\boldsymbol{x}_{a}\right) \cdot\left(\frac{\partial F}{\partial \boldsymbol{v}_{a}} \times \frac{\partial G}{\partial \boldsymbol{v}_{a}}\right) \\
& +\sum_{I, J=1}^{3 N_{1}} \sum_{K, L=1}^{3 N_{2}}\left(\frac{\partial F}{\partial \boldsymbol{e}_{I}}\left(\mathbb{M}_{1}^{-1}\right)_{I J} \mathbb{C}_{J K}^{\mathrm{T}} \frac{\partial G}{\partial \boldsymbol{b}_{L}}-\frac{\partial G}{\partial \boldsymbol{e}_{I}}\left(\mathbb{M}_{1}^{-1}\right)_{I J} \mathbb{C}_{J K}^{\mathrm{T}} \frac{\partial F}{\partial \boldsymbol{b}_{L}}\right),
\end{aligned}
$$

with the curl matrix $\mathbb{C}$ as given in (3.30). In order to express the semi-discrete Poisson bracket (4.21) in matrix form, we denote by $\Lambda^{1}(\boldsymbol{X})$ the $3 N_{p} \times 3 N_{1}$ matrix with generic term $\boldsymbol{\Lambda}_{I}^{1}\left(\boldsymbol{x}_{a}\right)$, where $1 \leqslant a \leqslant N_{p}$ and $1 \leqslant I \leqslant 3 N_{1}$, and by $\mathbb{B}(\boldsymbol{X}, \boldsymbol{b})$ the $3 N_{p} \times 3 N_{p}$ block diagonal matrix with generic block

$$
\widehat{\boldsymbol{B}}_{h}\left(\boldsymbol{x}_{a}, t\right)=\sum_{i=1}^{N_{2}}\left(\begin{array}{ccc}
0 & b_{i, 3}(t) \boldsymbol{\Lambda}_{i}^{2,3}\left(\boldsymbol{x}_{a}\right) & -b_{i, 2}(t) \boldsymbol{\Lambda}_{i}^{2,2}\left(\boldsymbol{x}_{a}\right) \\
-b_{i, 3}(t) \boldsymbol{\Lambda}_{i}^{2,3}\left(\boldsymbol{x}_{a}\right) & 0 & b_{i, 1}(t) \boldsymbol{\Lambda}_{i}^{2,1}\left(\boldsymbol{x}_{a}\right) \\
b_{i, 2}(t) \boldsymbol{\Lambda}_{i}^{2,2}\left(\boldsymbol{x}_{a}\right) & -b_{i, 1}(t) \boldsymbol{\Lambda}_{i}^{2,1}\left(\boldsymbol{x}_{a}\right) & 0
\end{array}\right) .
$$

Further, let us introduce a mass matrix $\boldsymbol{M}_{p}$ and a charge matrix $\boldsymbol{M}_{q}$ for the particles. Both are diagonal $N_{p} \times N_{p}$ matrices with elements $\left(\boldsymbol{M}_{p}\right)_{a a}=m_{a} w_{a}$ and $\left(\boldsymbol{M}_{q}\right)_{a a}=q_{a} w_{a}$, respectively. Additionally, we will need the $3 N_{p} \times 3 N_{p}$ matrices

$$
\mathbb{M}_{p}=\boldsymbol{M}_{p} \otimes \mathbb{I}_{3}, \quad \mathbb{M}_{q}=\boldsymbol{M}_{q} \otimes \mathbb{I}_{3},
$$

where $\mathbb{I}_{3}$ denotes the $3 \times 3$ identity matrix. This allows us to rewrite

$$
\begin{aligned}
& \sum_{a=1}^{N_{p}} \sum_{I=1}^{3 N_{2}} \frac{q_{a}}{m_{a}^{2} w_{a}} \boldsymbol{b}_{I}(t) \boldsymbol{\Lambda}_{I}^{2}\left(\boldsymbol{x}_{a}\right) \cdot\left(\frac{\partial F}{\partial \boldsymbol{v}_{a}} \times \frac{\partial G}{\partial \boldsymbol{v}_{a}}\right) \\
& =\sum_{a=1}^{N_{p}} \sum_{i=1}^{N_{2}} \sum_{\mu=1}^{3} \frac{q_{a}}{m_{a}^{2} w_{a}} b_{i, \mu}(t) \boldsymbol{\Lambda}_{i, \mu}^{2}\left(\boldsymbol{x}_{a}\right) \cdot\left(\frac{\partial F}{\partial \boldsymbol{v}_{a}} \times \frac{\partial G}{\partial \boldsymbol{v}_{a}}\right) \\
& =-\sum_{a=1}^{N_{p}} \frac{\partial F}{\partial \boldsymbol{v}_{a}} \frac{q_{a}}{m_{a}} \cdot \sum_{i=1}^{N_{2}} \sum_{\mu=1}^{3} b_{i, \mu}(t) \boldsymbol{\Lambda}_{i, \mu}^{2}\left(\boldsymbol{x}_{a}\right) \times \frac{1}{m_{a} w_{a}} \frac{\partial G}{\partial \boldsymbol{v}_{a}} \\
& =-\sum_{a=1}^{N_{p}} \frac{\partial F}{\partial \boldsymbol{v}_{a}} \frac{q_{a} w_{a}}{m_{a} w_{a}} \cdot \widehat{\boldsymbol{B}}_{h}\left(\boldsymbol{x}_{a}, t\right) \cdot \frac{1}{m_{a} w_{a}} \frac{\partial G}{\partial \boldsymbol{v}_{a}} \\
& =-\left(\frac{\partial F}{\partial \boldsymbol{V}}\right)^{\mathrm{T}} \mathbb{M}_{p}^{-1} \mathbb{M}_{q} \mathbb{B}(\boldsymbol{X}, \boldsymbol{b}) \mathbb{M}_{p}^{-1}\left(\frac{\partial G}{\partial \boldsymbol{V}}\right) \text {. }
\end{aligned}
$$

Here, the derivatives are represented by the $3 N_{p}$ vector

$$
\frac{\partial F}{\partial \boldsymbol{V}}=\left(\frac{\partial F}{\partial \boldsymbol{v}_{1}}, \ldots, \frac{\partial F}{\partial \boldsymbol{v}_{N_{p}}}\right)^{\mathrm{T}}=\left(\frac{\partial F}{\partial v_{1}^{1}}, \frac{\partial F}{\partial v_{1}^{2}}, \frac{\partial F}{\partial v_{1}^{3}}, \ldots, \frac{\partial F}{\partial v_{N_{p}}^{1}}, \frac{\partial F}{\partial v_{N_{p}}^{2}}, \frac{\partial F}{\partial v_{N_{p}}^{3}}\right)^{\mathrm{T}}
$$

and correspondingly for $\partial G / \partial \boldsymbol{V}, \partial F / \partial \boldsymbol{e}, \partial F / \partial \boldsymbol{b}$, etc. Thus, the discrete Poisson bracket (4.21) becomes 


$$
\begin{aligned}
\{F, G\}[\boldsymbol{X}, \boldsymbol{V}, \boldsymbol{e}, \boldsymbol{b}]= & \frac{\partial F}{\partial \boldsymbol{X}} \mathbb{M}_{p}^{-1} \frac{\partial G}{\partial \boldsymbol{V}}-\frac{\partial G}{\partial \boldsymbol{X}} \mathbb{M}_{p}^{-1} \frac{\partial F}{\partial \boldsymbol{V}} \\
& +\left(\frac{\partial F}{\partial \boldsymbol{V}}\right)^{\mathrm{T}} \mathbb{M}_{p}^{-1} \mathbb{M}_{q} \wedge^{1}(\boldsymbol{X})^{\mathrm{T}} \mathbb{M}_{1}^{-1}\left(\frac{\partial G}{\partial \boldsymbol{e}}\right) \\
& -\left(\frac{\partial F}{\partial \boldsymbol{e}}\right)^{\mathrm{T}} \mathbb{M}_{1}^{-1} \wedge^{1}(\boldsymbol{X}) \mathbb{M}_{q} \mathbb{M}_{p}^{-1}\left(\frac{\partial G}{\partial \boldsymbol{V}}\right) \\
& +\left(\frac{\partial F}{\partial \boldsymbol{V}}\right)^{\mathrm{T}} \mathbb{M}_{p}^{-1} \mathbb{M}_{q} \mathbb{B}(\boldsymbol{X}, \boldsymbol{b}) \mathbb{M}_{p}^{-1}\left(\frac{\partial G}{\partial \boldsymbol{V}}\right) \\
& +\left(\frac{\partial F}{\partial \boldsymbol{e}}\right)^{\mathrm{T}} \mathbb{M}_{1}^{-1} \mathbb{C}^{\mathrm{T}}\left(\frac{\partial G}{\partial \boldsymbol{b}}\right)-\left(\frac{\partial F}{\partial \boldsymbol{b}}\right)^{\mathrm{T}} \mathbb{C M}_{1}^{-1}\left(\frac{\partial G}{\partial \boldsymbol{e}}\right) .
\end{aligned}
$$

The action of this bracket on two functions $F$ and $G$ can also be expressed as

$$
\{F, G\}=D F^{\mathrm{T}} \mathbb{J}(\boldsymbol{u}) D G,
$$

denoting by $D$ the derivative with respect to the dynamical variables

$$
\boldsymbol{u}=(\boldsymbol{X}, \boldsymbol{V}, \boldsymbol{e}, \boldsymbol{b})^{\mathrm{T}},
$$

and by $\mathbb{J}$ the Poisson matrix, given by

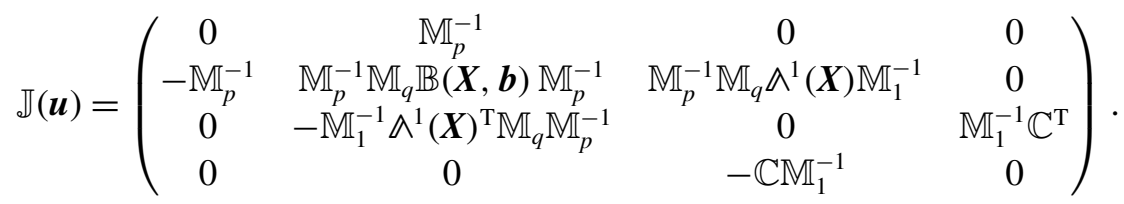

We immediately see that $\mathbb{J}(\boldsymbol{u})$ is anti-symmetric, but it remains to be shown that it satisfies the Jacobi identity.

\subsection{Jacobi identity}

The discrete Poisson bracket (4.26) satisfies the Jacobi identity if and only if the following condition holds (see e.g. (Morrison 1998, § IV) or (Hairer, Lubich \& Wanner 2006, § VII.2, Lemma 2.3)):

$$
\sum_{l}\left(\frac{\partial \mathbb{J}_{i j}(\boldsymbol{u})}{\partial u_{l}} \mathbb{J}_{l k}(\boldsymbol{u})+\frac{\partial \mathbb{J}_{j k}(\boldsymbol{u})}{\partial u_{l}} \mathbb{J}_{l i}(\boldsymbol{u})+\frac{\partial \mathbb{J}_{k i}(\boldsymbol{u})}{\partial u_{l}} \mathbb{J}_{l j}(\boldsymbol{u})\right)=0 \quad \text { for all } i, j, k .
$$

Here, all indices $i, j, k, l$ run from 1 to $6 N_{p}+3 N_{1}+3 N_{2}$. To simplify the verification of (4.30), we start by identifying blocks of the Poisson matrix $\mathbb{J}$ whose elements contribute to the above condition. Therefore, we write

$$
\mathbb{J}(\boldsymbol{u})=\left(\begin{array}{llll}
\boldsymbol{J}_{11}(\boldsymbol{u}) & \boldsymbol{J}_{12}(\boldsymbol{u}) & \boldsymbol{J}_{13}(\boldsymbol{u}) & \boldsymbol{J}_{14}(\boldsymbol{u}) \\
\boldsymbol{J}_{21}(\boldsymbol{u}) & \boldsymbol{J}_{22}(\boldsymbol{u}) & \boldsymbol{J}_{23}(\boldsymbol{u}) & \boldsymbol{J}_{24}(\boldsymbol{u}) \\
\boldsymbol{J}_{31}(\boldsymbol{u}) & \boldsymbol{J}_{32}(\boldsymbol{u}) & \boldsymbol{J}_{33}(\boldsymbol{u}) & \boldsymbol{J}_{34}(\boldsymbol{u}) \\
\boldsymbol{J}_{41}(\boldsymbol{u}) & \boldsymbol{J}_{42}(\boldsymbol{u}) & \boldsymbol{J}_{43}(\boldsymbol{u}) & \boldsymbol{J}_{44}(\boldsymbol{u})
\end{array}\right)=\left(\begin{array}{cccc}
0 & \boldsymbol{J}_{12} & 0 & 0 \\
\boldsymbol{J}_{21} & \boldsymbol{J}_{22}(\boldsymbol{X}, \boldsymbol{b}) & \boldsymbol{J}_{23}(\boldsymbol{X}) & 0 \\
0 & \boldsymbol{J}_{32}(\boldsymbol{X}) & 0 & \boldsymbol{J}_{34} \\
0 & 0 & \boldsymbol{J}_{43} & 0
\end{array}\right) .
$$

The Poisson matrix $\mathbb{J}$ only depends on $\boldsymbol{X}$ and $\boldsymbol{b}$, so in (4.30) we only need to sum $l$ over the corresponding indices, $1 \leqslant l \leqslant 3 N_{p}$ and $6 N_{p}+3 N_{1}<l \leqslant 6 N_{p}+3 N_{1}+3 N_{2}$, 
respectively. Considering the terms $\mathbb{J}_{l i}(\boldsymbol{u}), \mathbb{J}_{l j}(\boldsymbol{u})$ and $\mathbb{J}_{l k}(\boldsymbol{u})$, we see that in the aforementioned index ranges for $l$, only $\boldsymbol{J}_{12}=\mathbb{M}_{p}^{-1}$ and $\boldsymbol{J}_{43}=-\mathbb{M}_{2}^{-1} \mathbb{C M}_{1}^{-1}$ are non-vanishing, so that we have to account only for those two blocks, i.e. for $1 \leqslant l \leqslant 3 N_{p}$ we only need to consider $3 N_{p}<i, j, k \leqslant 6 N_{p}$ and for $6 N_{p}+3 N_{1}<$ $l \leqslant 6 N_{p}+3 N_{1}+3 N_{2}$ we only need to consider $6 N_{p}<i, j, k \leqslant 6 N_{p}+3 N_{1}$. Note that $\boldsymbol{J}_{12}$ is a diagonal matrix, therefore $\left(\boldsymbol{J}_{12}\right)_{a b}=\left(\boldsymbol{J}_{12}\right)_{a a} \delta_{a b}$ with $1 \leqslant a, b \leqslant N_{p}$. Further, only $\boldsymbol{J}_{22}, \boldsymbol{J}_{23}$ and $\boldsymbol{J}_{32}$ depend on $\boldsymbol{b}$ and/or $\boldsymbol{X}$, so only those blocks have to be considered when computing derivatives with respect to $\boldsymbol{u}$. In summary, we obtain two conditions. The contributions involving $\boldsymbol{J}_{22}$ and $\boldsymbol{J}_{12}$ are

$$
\begin{aligned}
& \sum_{a=1}^{3 N_{p}}\left(\frac{\partial\left(\boldsymbol{J}_{22}(\boldsymbol{X}, \boldsymbol{b})\right)_{b c}}{\partial \boldsymbol{X}_{a}}\left(\boldsymbol{J}_{12}\right)_{a d}+\frac{\partial\left(\boldsymbol{J}_{22}(\boldsymbol{X}, \boldsymbol{b})\right)_{c d}}{\partial \boldsymbol{X}_{a}}\left(\boldsymbol{J}_{12}\right)_{a b}\right. \\
& \left.\quad+\frac{\partial\left(\boldsymbol{J}_{22}(\boldsymbol{X}, \boldsymbol{b})\right)_{d b}}{\partial \boldsymbol{X}_{a}}\left(\boldsymbol{J}_{12}\right)_{a c}\right)=0,
\end{aligned}
$$

for $1 \leqslant b, c, d \leqslant 3 N_{p}$, which corresponds to (4.30) for $3 N_{p}<i, j, k \leqslant 6 N_{p}$. Inserting the actual values for $\boldsymbol{J}_{12}$ and $\boldsymbol{J}_{22}$ and using that $\mathbb{M}_{p}$ is diagonal, equation (4.32) becomes

$$
\begin{gathered}
\frac{\partial\left(\mathbb{M}_{p}^{-1} \mathbb{M}_{q} \mathbb{B}(\boldsymbol{X}, \boldsymbol{b}) \mathbb{M}_{p}^{-1}\right)_{b c}}{\partial \boldsymbol{X}_{d}}\left(\mathbb{M}_{p}^{-1}\right)_{d d}+\frac{\partial\left(\mathbb{M}_{p}^{-1} \mathbb{M}_{q} \mathbb{B}(\boldsymbol{X}, \boldsymbol{b}) \mathbb{M}_{p}^{-1}\right)_{c d}}{\partial \boldsymbol{X}_{b}}\left(\mathbb{M}_{p}^{-1}\right)_{b b} \\
+\frac{\partial\left(\mathbb{M}_{p}^{-1} \mathbb{M}_{q} \mathbb{B}(\boldsymbol{X}, \boldsymbol{b}) \mathbb{M}_{p}^{-1}\right)_{d b}}{\partial \boldsymbol{X}_{c}}\left(\mathbb{M}_{p}^{-1}\right)_{c c}=0 .
\end{gathered}
$$

All outer indices of this expression belong to the inverse matrix $\mathbb{M}_{p}^{-1}$. As this matrix is constant, symmetric and positive definite, we can contract the above expression with $\mathbb{M}_{p}$ on all indices, to obtain

$$
\frac{\partial\left(\mathbb{M}_{q} \mathbb{B}(\boldsymbol{X}, \boldsymbol{b})\right)_{b c}}{\partial \boldsymbol{X}_{d}}+\frac{\partial\left(\mathbb{M}_{q} \mathbb{B}(\boldsymbol{X}, \boldsymbol{b})\right)_{c d}}{\partial \boldsymbol{X}_{b}}+\frac{\partial\left(\mathbb{M}_{q} \mathbb{B}(\boldsymbol{X}, \boldsymbol{b})\right)_{d b}}{\partial \boldsymbol{X}_{c}}=0, \quad 1 \leqslant b, c, d \leqslant 3 N_{p} .
$$

If in the first term of (4.34) one picks a particular index $k$, then this selects the $\sigma$ component of the position $\boldsymbol{x}_{a}$ of some particle. At the same time, in the second and third terms, this selects a block of (4.22), which is evaluated at the same particle position $\boldsymbol{x}_{a}$. This means that the only non-vanishing contributions in (4.34) will be those indexed by $b$ and $c$ with $\boldsymbol{X}_{b}$ and $\boldsymbol{X}_{c}$ corresponding to components $\mu$ and $v$ of the same particle position $\boldsymbol{x}_{a}$. Therefore, the condition (4.22) reduces further to

$$
q_{a}\left(\frac{\partial \widehat{B}_{\mu \nu}\left(\boldsymbol{x}_{a}\right)}{\partial x_{a}^{\sigma}}+\frac{\partial \widehat{\boldsymbol{B}}_{\nu \sigma}\left(\boldsymbol{x}_{a}\right)}{\partial x_{a}^{\mu}}+\frac{\partial \widehat{\boldsymbol{B}}_{\sigma \mu}\left(\boldsymbol{x}_{a}\right)}{\partial x_{a}^{v}}\right)=0, \quad 1 \leqslant a \leqslant N_{p}, 1 \leqslant \mu, \nu, \sigma \leqslant 3,
$$

where $\widehat{B}_{\mu \nu}$ denotes the components of the matrix in (4.22). When all three indices are equal, this corresponds to diagonal terms of the matrix $\widehat{\boldsymbol{B}}_{h}\left(\boldsymbol{x}_{a}, t\right)$ which vanish. When two of the three are equal, it cancels because of the skew symmetry of the same matrix and for all three indices distinct, this condition corresponds to $\operatorname{div} \boldsymbol{B}_{h}=0$. Choosing initial conditions such that $\operatorname{div} \boldsymbol{B}_{h}(\boldsymbol{x}, 0)=0$ and using a discrete deRham complex guarantees $\operatorname{div} \boldsymbol{B}_{h}(\boldsymbol{x}, t)=0$ for all times $t$. Note that this was to be expected, because it is the discrete version of the $\operatorname{div} \boldsymbol{B}=0$ condition for the continuous Poisson bracket (2.8) (Morrison 1982). Further note that in the discrete case, just as in the 
continuous case, the Jacobi identity requires the magnetic field to be divergence free, but it is not required to be the curl of some vector potential. In the language of differential forms this is to say that the magnetic field as a 2-form needs to be closed but does not need to be exact.

From the contributions involving $\boldsymbol{J}_{22}, \boldsymbol{J}_{23}, \boldsymbol{J}_{32}$ and $\boldsymbol{J}_{43}$ we have

$$
\begin{gathered}
\sum_{a=1}^{3 N_{p}}\left(\frac{\partial\left(\boldsymbol{J}_{23}(\boldsymbol{X}, \boldsymbol{b})\right)_{c I}}{\partial \boldsymbol{X}_{a}}\left(\boldsymbol{J}_{12}\right)_{a b}+\frac{\partial\left(\boldsymbol{J}_{32}(\boldsymbol{X}, \boldsymbol{b})\right)_{I b}}{\partial \boldsymbol{X}_{a}}\left(\boldsymbol{J}_{12}\right)_{a c}\right) \\
+\sum_{J=1}^{N_{2}} \frac{\partial\left(\boldsymbol{J}_{22}(\boldsymbol{X}, \boldsymbol{b})\right)_{b c}}{\partial \boldsymbol{b}_{J}}\left(\boldsymbol{J}_{43}\right)_{J I}=0
\end{gathered}
$$

for $1 \leqslant b, c \leqslant 3 N_{p}$ and $1 \leqslant I \leqslant 3 N_{1}$, which corresponds to (4.30) for $3 N_{p}<i, j \leqslant 6 N_{p}<$ $k \leqslant 6 N_{p}+3 N_{1}$. Writing out (4.36) and using that $\mathbb{M}_{p}$ is diagonal gives

$$
\begin{gathered}
\frac{\partial\left(\mathbb{M}_{1}^{-1} \bigwedge^{1}(\boldsymbol{X})^{\mathrm{T}} \mathbb{M}_{q} \mathbb{M}_{p}^{-1}\right)_{I b}}{\partial \boldsymbol{X}_{c}}\left(\mathbb{M}_{p}^{-1}\right)_{c c}-\frac{\partial\left(\mathbb{M}_{p}^{-1} \mathbb{M}_{q} \bigwedge^{1}(\boldsymbol{X}) \mathbb{M}_{1}^{-1}\right)_{c I}}{\partial \boldsymbol{X}_{b}}\left(\mathbb{M}_{p}^{-1}\right)_{b b} \\
=\sum_{J=1}^{N_{2}} \frac{\partial\left(\mathbb{M}_{p}^{-1} \mathbb{M}_{q} \mathbb{B}(\boldsymbol{X}, \boldsymbol{b}) \mathbb{M}_{p}^{-1}\right)_{b c}}{\partial \boldsymbol{b}_{J}}\left(\mathbb{C M}_{1}^{-1}\right)_{J I}
\end{gathered}
$$

Again, we can contract this with the matrix $\mathbb{M}_{p}$ on the indices $b$ and $c$, in order to remove $\mathbb{M}_{p}^{-1}$, and with $\mathbb{M}_{1}$ on the index $I$, in order to remove $\mathbb{M}_{1}^{-1}$. Similarly, $\mathbb{M}_{q}$ can be removed by contracting with $\mathbb{M}_{q}^{-1}$, noting that this matrix is constant and therefore commutes with the curl. This results in the simplified condition

$$
\frac{\partial \bigwedge_{b I}^{1}(\boldsymbol{X})}{\partial \boldsymbol{X}_{c}}-\frac{\partial \bigwedge_{c I}^{1}(\boldsymbol{X})}{\partial \boldsymbol{X}_{b}}=\sum_{J=1}^{N_{2}} \frac{\partial \mathbb{B}_{b c}(\boldsymbol{X}, \boldsymbol{b})}{\partial \boldsymbol{b}_{J}} \mathbb{C}_{J I}
$$

The $\boldsymbol{b}_{J}$ derivative of $\mathbb{B}$ results in the $3 N_{p} \times 3 N_{p}$ block diagonal matrix $\bigwedge_{J}^{2}(\boldsymbol{X})$ with generic block

$$
\widehat{\boldsymbol{\Lambda}}_{J}^{2}\left(\boldsymbol{x}_{a}\right)=\left(\begin{array}{ccc}
0 & \boldsymbol{\Lambda}_{J}^{2,3}\left(\boldsymbol{x}_{a}\right) & -\boldsymbol{\Lambda}_{J}^{2,2}\left(\boldsymbol{x}_{a}\right) \\
-\boldsymbol{\Lambda}_{J}^{2,3}\left(\boldsymbol{x}_{a}\right) & 0 & \boldsymbol{\Lambda}_{J}^{2,1}\left(\boldsymbol{x}_{a}\right) \\
\boldsymbol{\Lambda}_{J}^{2,2}\left(\boldsymbol{x}_{a}\right) & -\boldsymbol{\Lambda}_{J}^{2,1}\left(\boldsymbol{x}_{a}\right) & 0
\end{array}\right)
$$

so that (4.38) becomes

$$
\frac{\partial\left(\boldsymbol{\Lambda}^{1}(\boldsymbol{X})^{\mathrm{T}} \mathbb{M}_{q}\right)_{I b}}{\partial \boldsymbol{X}_{c}}-\frac{\partial\left(\mathbb{M}_{q} \bigwedge^{1}(\boldsymbol{X})\right)_{c I}}{\partial \boldsymbol{X}_{b}}=\sum_{J=1}^{N_{2}} \mathbb{C}_{I J}^{\mathrm{T}}\left(\mathbb{M}_{q} \bigwedge_{J}^{2}(\boldsymbol{X})\right)_{b c}
$$

This condition can be compactly written as

$$
\operatorname{curl} \AA^{1}(\boldsymbol{X})=\bigwedge^{2}(\boldsymbol{X}) \mathbb{C}
$$

That the charge matrices $\mathbb{M}_{q}$ can be eliminated can be seen as follows. Similarly as before, picking a particular index $c$ in the first term selects the $v$ component of the position $\boldsymbol{x}_{a}$ of some particle. At the same time, in the second term, this selects the $v$ 
component of $\boldsymbol{\Lambda}^{1}$, evaluated at the same particle position $\boldsymbol{x}_{a}$. The only non-vanishing derivative of this term is therefore with respect to components of the same particle position, so that $\boldsymbol{X}_{b}$ denotes the $\mu$ component of $\boldsymbol{x}_{a}$. Hence, condition (4.40) simplifies to

$$
q_{a}\left(\frac{\partial \boldsymbol{\Lambda}_{I}^{1, \mu}\left(\boldsymbol{x}_{a}\right)}{\partial x_{a}^{\nu}}-\frac{\partial \boldsymbol{\Lambda}_{I}^{1, \nu}\left(\boldsymbol{x}_{a}\right)}{\partial x_{a}^{\mu}}\right)=q_{a} \sum_{J=1}^{N_{2}} \mathbb{C}_{I J}^{\mathrm{T}}\left(\widehat{\boldsymbol{\Lambda}}_{J}^{2}\left(\boldsymbol{x}_{a}\right)\right)_{\mu \nu},
$$

for $1 \leqslant a \leqslant N_{p}, 1 \leqslant \mu, v \leqslant 3$ and $1 \leqslant I \leqslant 3 N_{1}$. The charge $q_{a}$ is the same on both sides and can therefore be removed. This conditions states how the curl of the 1-form basis, evaluated at some particle's position, is expressed in the 2-form basis, evaluated at the same particle's position, using the curl matrix $\mathbb{C}$. For spaces which build a deRham complex, this is always satisfied. This concludes the verification of condition (4.30) for the Jacobi identity to hold for the discrete bracket (4.26).

\subsection{Discrete Hamiltonian and equations of motion}

The Hamiltonian is discretized by inserting (4.15) and (3.25) into (2.11),

$$
\begin{aligned}
H(\boldsymbol{V}, \boldsymbol{e}, \boldsymbol{b}) \equiv & \mathcal{H}\left[f_{h}, \boldsymbol{E}_{h}, \boldsymbol{B}_{h}\right] \\
= & \frac{1}{2} \int|\boldsymbol{v}|^{2} \sum_{a=1}^{N_{p}} m_{a} w_{a} \delta\left(\boldsymbol{x}-\boldsymbol{x}_{a}(t)\right) \delta\left(\boldsymbol{v}-\boldsymbol{v}_{a}(t)\right) \mathrm{d} \boldsymbol{x} \mathrm{d} \boldsymbol{v} \\
& +\frac{1}{2} \int\left[\left|\sum_{I=1}^{3 N_{1}} \boldsymbol{e}_{I}(t) \boldsymbol{\Lambda}_{I}^{1}(\boldsymbol{x})\right|^{2}+\left|\sum_{J=1}^{3 N_{2}} \boldsymbol{b}_{J}(t) \boldsymbol{\Lambda}_{J}^{2}(\boldsymbol{x})\right|^{2}\right] \mathrm{d} \boldsymbol{x},
\end{aligned}
$$

which in matrix notation becomes

$$
H=\frac{1}{2} \boldsymbol{V}^{\mathrm{T}} \mathbb{M}_{p} \boldsymbol{V}+\frac{1}{2} \boldsymbol{e}^{\mathrm{T}} \mathbb{M}_{1} \boldsymbol{e}+\frac{1}{2} \boldsymbol{b}^{\mathrm{T}} \mathbb{M}_{2} \boldsymbol{b}
$$

The semi-discrete equations of motion are given by

$$
\dot{\boldsymbol{X}}=\{\boldsymbol{X}, H\}, \quad \dot{\boldsymbol{V}}=\{\boldsymbol{V}, H\}, \quad \dot{\boldsymbol{e}}=\{\boldsymbol{e}, H\}, \quad \dot{\boldsymbol{b}}=\{\boldsymbol{b}, H\},
$$

which are equivalent to

$$
\dot{\boldsymbol{u}}=\mathbb{J}(\boldsymbol{u}) D H(\boldsymbol{u}) .
$$

With $D H(\boldsymbol{u})=\left(0, \mathbb{M}_{p} \boldsymbol{V}, \mathbb{M}_{1} \boldsymbol{e}, \mathbb{M}_{2} \boldsymbol{b}\right)^{\mathrm{T}}$, we obtain

$$
\begin{aligned}
\dot{\boldsymbol{X}} & =\boldsymbol{V}, \\
\dot{\boldsymbol{V}} & =\mathbb{M}_{p}^{-1} \mathbb{M}_{q}\left(\boldsymbol{\Lambda}^{1}(\boldsymbol{X}) \boldsymbol{e}+\mathbb{B}(\boldsymbol{X}, \boldsymbol{b}) \boldsymbol{V}\right), \\
\dot{\boldsymbol{e}} & =\mathbb{M}_{1}^{-1}\left(\mathbb{C}^{\mathrm{T}} \mathbb{M}_{2} \boldsymbol{b}(t)-\mathbb{A}^{1}(\boldsymbol{X})^{\mathrm{T}} \mathbb{M}_{q} \boldsymbol{V}\right), \\
\dot{\boldsymbol{b}} & =-\mathbb{C} \boldsymbol{e}(t),
\end{aligned}
$$

where the first two equations describe the particle dynamics and the last two equations describe the evolution of the electromagnetic fields. Note that $\mathbb{M}_{p}$ and $\mathbb{M}_{q}$ are diagonal matrices and that $\mathbb{M}_{p}^{-1} \mathbb{M}_{q}$ is nothing but the factor $q_{a} / m_{a}$ for the particle labelled by $a$. The purpose of introducing these matrices is solely to obtain a compact notation and to display the Poisson structure of the semi-discrete system. However, these matrices are neither explicitly constructed nor is there a need to invert them. 


\subsection{Discrete Gauss' law}

Multiplying $(4.47 c)$ by $\mathbb{G}^{\mathrm{T}} \mathbb{M}_{1}$ on the left, we get

$$
\mathbb{G}^{\mathrm{T}} \mathrm{M}_{1} \dot{\boldsymbol{e}}(t)=\mathbb{G}^{\mathrm{T}} \mathbb{C}^{\mathrm{T}} \mathbb{M}_{2} \boldsymbol{b}(t)-\mathbb{G}^{\mathrm{T}} \bigwedge^{1}(\boldsymbol{X}(t))^{\mathrm{T}} \mathbb{M}_{q} \boldsymbol{V}(t) .
$$

As $\mathbb{C} \mathbb{G}=0$ from (3.37), the first term on the right-hand side vanishes. Observe that

$$
\wedge^{1}(\boldsymbol{X}) \mathbb{G} \psi=\operatorname{grad} \bigwedge^{0}(\boldsymbol{X}) \psi \quad \forall \psi \in \mathbb{R}^{N_{0}},
$$

and using $\mathrm{d} \boldsymbol{x}_{a} / \mathrm{d} t=\boldsymbol{v}_{a}$ we find that

$$
\frac{\mathrm{d} \Psi_{h}\left(\boldsymbol{x}_{a}(t)\right)}{\mathrm{d} t}=\frac{\mathrm{d} \boldsymbol{x}_{a}(t)}{\mathrm{d} t} \cdot \operatorname{grad} \Psi_{h}\left(\boldsymbol{x}_{a}(t)\right)=\boldsymbol{v}_{a}(t) \cdot \operatorname{grad} \Psi_{h}\left(\boldsymbol{x}_{a}(t)\right),
$$

for any $\Psi_{h} \in V_{0}$ with $\operatorname{grad} \Psi_{h} \in V_{1}$, so that we obtain

$$
\mathbb{G}^{\mathrm{T}} \mathbb{M}_{1} \dot{\boldsymbol{e}}=-\mathbb{G}^{\mathrm{T}} \boldsymbol{\Lambda}^{1}(\boldsymbol{X})^{\mathrm{T}} \mathbb{M}_{q} \boldsymbol{V}=-\left(\operatorname{grad} \boldsymbol{\iota}^{0}(\boldsymbol{X})\right)^{\mathrm{T}} \mathbb{M}_{q} \frac{\mathrm{d} \boldsymbol{X}}{\mathrm{d} t}=-\frac{\mathrm{d} \boldsymbol{\iota}^{0}(\boldsymbol{X})^{\mathrm{T}}}{\mathrm{d} t} \mathbb{M}_{q} \mathbb{1}_{N_{p}},
$$

where $\mathbb{1}_{N_{p}}$ denotes the column vector with $N_{p}$ terms all being unity, needed for the sum over the particles when there is no velocity vector. This shows that the discrete Gauss' law is conserved,

$$
\mathbb{G}^{\mathrm{T}} \mathbb{M}_{1} \boldsymbol{e}=-\boldsymbol{\Lambda}^{0}(\boldsymbol{X})^{\mathrm{T}} \mathbb{M}_{q} \mathbb{1}_{N_{p}} .
$$

Moreover, note that (4.51) also contains a discrete version of the continuity equation (2.7),

$$
\frac{\mathrm{d} \varrho}{\mathrm{d} t}+\mathbb{G}^{\mathrm{T}} \boldsymbol{j}=0,
$$

with the discrete charge and current density given by

$$
\varrho=-\boldsymbol{\Lambda}^{0}(\boldsymbol{X})^{\mathrm{T}} \mathbb{M}_{q} \mathbb{1}_{N_{p}}, \quad \boldsymbol{j}=\boldsymbol{\Lambda}^{1}(\boldsymbol{X})^{\mathrm{T}} \mathbb{M}_{q} \boldsymbol{V} .
$$

The conservation of this relation in the fully discrete system depends on the choice of the time stepping scheme.

\subsection{Discrete Casimir invariants}

Let us now find the Casimir invariants of the semi-discrete Poisson structure. In order to obtain the discrete Casimir invariants, we assume that the discrete spaces in (3.9) not only form a complex, so that $\operatorname{Im}($ grad $) \subseteq \operatorname{Ker}(\operatorname{curl})$ and $\operatorname{Im}($ curl $) \subseteq \operatorname{Ker}(\operatorname{div})$, but that they form an exact sequence, so that $\operatorname{Im}(\operatorname{grad})=\operatorname{Ker}(\operatorname{curl})$ and $\operatorname{Im}(\operatorname{curl})=\operatorname{Ker}(\operatorname{div})$, i.e. at each node in (3.9), the image of the previous operator is not only a subset of the kernel of the next operator but is exactly equal to the kernel of the next operator. We will then see that this requirement is not necessary for the identified functionals to be valid Casimir invariants. However, it is a useful assumption in their identification.

The Casimir invariants of the semi-discrete system are functions $C(\boldsymbol{X}, \boldsymbol{V}, \boldsymbol{e}, \boldsymbol{b})$ such that $\{C, F\}=0$ for any function $F$. In terms of the Poisson matrix $\mathbb{J}$, this can be expressed as $\mathbb{J}(\boldsymbol{u}) D C(\boldsymbol{u})=0$. Upon writing this for each of the lines of $\mathbb{J}$ of (4.29), we find for the first line

$$
\mathbb{M}_{p}^{-1} D_{V} C=0 .
$$


This implies that $C$ does not depend on $\boldsymbol{V}$, which we shall use in the sequel. Then the third line simply becomes

$$
\mathbb{M}_{1}^{-1} \mathbb{C}^{\mathrm{T}} D_{b} C=0 \Rightarrow D_{b} C \in \operatorname{Ker}\left(\mathbb{C}^{\mathrm{T}}\right)
$$

Then, because of the exact sequence property, there exists $\bar{b} \in \mathbb{R}^{N_{3}}$ such that $D_{b} C=$ $\mathbb{D}^{\mathrm{T}} \overline{\boldsymbol{b}}$. Hence all functions of the form

$$
C(\boldsymbol{b})=\boldsymbol{b}^{\mathrm{T}} \mathbb{D}^{\mathrm{T}} \overline{\boldsymbol{b}}=\overline{\boldsymbol{b}}^{\mathrm{T}} \mathbb{D} \boldsymbol{b}, \quad \overline{\boldsymbol{b}} \in \mathbb{R}^{N_{3}}
$$

are Casimirs, which means that $\mathbb{D} \boldsymbol{b}$, the matrix form of $\operatorname{div} \boldsymbol{B}_{h}$, is conserved.

The fourth line, using that $C$ does not depend on $\boldsymbol{V}$, becomes

$$
\mathbb{C M}_{1}^{-1} D_{e} C=0 \quad \Rightarrow \quad \mathbb{M}_{1}^{-1} D_{e} C \in \operatorname{Ker}(\mathbb{C}) \text {. }
$$

Because of the exact sequence property there exists $\overline{\boldsymbol{e}} \in \mathbb{R}^{N_{1}}$, such that $D_{\boldsymbol{e}} C=\mathbb{M}_{1} \mathbb{G} \overline{\boldsymbol{e}}$. Finally, the second line couples $\boldsymbol{e}$ and $\boldsymbol{X}$ and reads, upon multiplying by $M_{p}$,

$$
D_{X} C=\mathbb{M}_{q} \lambda^{1}(X) \mathbb{M}_{1}^{-1} D_{e} C=\mathbb{M}_{q} \lambda^{1}(\boldsymbol{X}) \mathbb{G} \overline{\boldsymbol{e}}=\mathbb{M}_{q} \operatorname{grad} \Lambda_{0}(\boldsymbol{X}) \overline{\boldsymbol{e}},
$$

using the expression for $D_{e} C$ derived previously and (4.49). It follows that all functions of the form

$$
C(\boldsymbol{X}, \boldsymbol{e})=\mathbb{1}_{N}^{\mathrm{T}} \mathbb{M}_{q} \lambda^{0}(\boldsymbol{X}) \overline{\boldsymbol{e}}+\boldsymbol{e}^{\mathrm{T}} \mathbb{M}_{1} \mathbb{G} \overline{\boldsymbol{e}}=\overline{\boldsymbol{e}}^{\mathrm{T}} \boldsymbol{\Lambda}^{0}(\boldsymbol{X})^{\mathrm{T}} \mathbb{M}_{q} \mathbb{1}_{N}+\overline{\boldsymbol{e}}^{\mathrm{T}} \mathbb{G}^{\mathrm{T}} \mathbb{M}_{1} \boldsymbol{e}, \quad \overline{\boldsymbol{e}} \in \mathbb{R}^{N_{0}},
$$

are Casimirs, so that $\mathbb{G}^{\mathrm{T}} \mathbb{M}_{1} \boldsymbol{e}+\boldsymbol{\Lambda}^{0}(\boldsymbol{X})^{\mathrm{T}} \mathbb{M}_{q} \mathbb{1}_{N}$ is conserved. This is the matrix form of Gauss' law (4.52).

Having identified the discrete Casimir invariants (4.57) and (4.60), it is easy to see by plugging them into the discrete Poisson bracket (4.26) that they are valid Casimir invariants, even if the deRham complex is not exact, because all that is needed for $\mathbb{J}(\boldsymbol{u}) D C(\boldsymbol{u})=0$ is the complex property $\operatorname{Im} \mathbb{D}^{\mathrm{T}} \subseteq \operatorname{Ker} \mathbb{C}^{\mathrm{T}}$ and $\operatorname{grad} \wedge^{0}(\boldsymbol{X})=\bigwedge^{1}(\boldsymbol{X}) \mathbb{G}$.

\section{Hamiltonian splitting}

Following Crouseilles et al. (2015), He et al. (2015), Qin et al. (2015), we split the discrete Hamiltonian (4.44) into three parts,

$$
H=H_{p}+H_{E}+H_{B}
$$

with

$$
H_{p}=\frac{1}{2} \boldsymbol{V}^{\mathrm{T}} \mathbb{M}_{p} \boldsymbol{V}, \quad H_{E}=\frac{1}{2} \boldsymbol{e}^{\mathrm{T}} \mathbb{M}_{1} \boldsymbol{e}, \quad H_{B}=\frac{1}{2} \boldsymbol{b}^{\mathrm{T}} \mathbb{M}_{2} \boldsymbol{b} .
$$

Writing $\boldsymbol{u}=(\boldsymbol{X}, \boldsymbol{V}, \boldsymbol{e}, \boldsymbol{b})^{\mathrm{T}}$, we split the discrete Vlasov-Maxwell equations (4.47) into three subsystems,

$$
\dot{\boldsymbol{u}}=\left\{\boldsymbol{u}, H_{p}\right\}, \quad \dot{\boldsymbol{u}}=\left\{\boldsymbol{u}, H_{E}\right\}, \quad \dot{\boldsymbol{u}}=\left\{\boldsymbol{u}, H_{B}\right\}
$$

The exact solution to each of these subsystems will constitute a Poisson map. Because a composition of Poisson maps is itself a Poisson map, we can construct Poisson structure-preserving integration methods for the Vlasov-Maxwell system by composition of the exact solutions of each of the subsystems. 
5.1. Solution of the subsystems

The discrete equations of motion for $H_{E}$ are

$$
\begin{aligned}
\dot{\boldsymbol{X}} & =0, \\
\mathbb{M}_{p} \dot{\boldsymbol{V}} & =\mathbb{M}_{q} \wedge^{1}(\boldsymbol{X}) \boldsymbol{e}, \\
\dot{\boldsymbol{e}} & =0, \\
\dot{\boldsymbol{b}} & =-\mathbb{C} \boldsymbol{e}(t) .
\end{aligned}
$$

For initial conditions $(\boldsymbol{X}(0), \boldsymbol{V}(0), \boldsymbol{e}(0), \boldsymbol{b}(0))$ the exact solutions at time $\Delta t$ are given by the map $\varphi_{\Delta t, E}$ defined as

$$
\begin{aligned}
\boldsymbol{X}(\Delta t) & =\boldsymbol{X}(0) \\
\mathbb{M}_{p} \boldsymbol{V}(\Delta t) & =\mathbb{M}_{p} \boldsymbol{V}(0)+\Delta t \mathbb{M}_{q} \wedge^{1}(\boldsymbol{X}(0)) \boldsymbol{e}(0), \\
\boldsymbol{e}(\Delta t) & =\boldsymbol{e}(0) \\
\boldsymbol{b}(\Delta t) & =\boldsymbol{b}(0)-\Delta t \mathbb{C} \boldsymbol{e}(0)
\end{aligned}
$$

The discrete equations of motion for $H_{B}$ are

$$
\begin{aligned}
\dot{\boldsymbol{X}} & =0, \\
\dot{\boldsymbol{V}} & =0, \\
\mathbb{M}_{1} \dot{\boldsymbol{e}} & =\mathbb{C}^{\mathrm{T}} \mathbb{M}_{2} \boldsymbol{b}(t), \\
\dot{\boldsymbol{b}} & =0
\end{aligned}
$$

For initial conditions $(\boldsymbol{X}(0), \boldsymbol{V}(0), \boldsymbol{e}(0), \boldsymbol{b}(0))$ the exact solutions at time $\Delta t$ are given by the map $\varphi_{\Delta t, B}$ defined as

$$
\begin{aligned}
\boldsymbol{X}(\Delta t) & =\boldsymbol{X}(0) \\
\boldsymbol{V}(\Delta t) & =\boldsymbol{V}(0) \\
\mathbb{M}_{1} \boldsymbol{e}(\Delta t) & =\mathbb{M}_{1} \boldsymbol{e}(0)+\Delta t \mathbb{C}^{\mathrm{T}} \mathbb{M}_{2} \boldsymbol{b}(0) \\
\boldsymbol{b}(\Delta t) & =\boldsymbol{b}(0)
\end{aligned}
$$

The discrete equations of motion for $H_{p}$ are

$$
\begin{aligned}
\dot{\boldsymbol{X}} & =\boldsymbol{V}, \\
\mathbb{M}_{p} \dot{\boldsymbol{V}} & =\mathbb{M}_{q} \mathbb{B}(\boldsymbol{X}, \boldsymbol{b}) \boldsymbol{V}, \\
\mathbb{M}_{1} \dot{\boldsymbol{e}} & =-\boldsymbol{\Lambda}^{1}(\boldsymbol{X})^{\mathrm{T}} \mathbb{M}_{q} \boldsymbol{V}, \\
\dot{\boldsymbol{b}} & =0 .
\end{aligned}
$$

For general magnetic field coefficients $\boldsymbol{b}$, this system cannot be exactly integrated (He et al. 2015). Note that each component $\dot{\boldsymbol{V}}_{\mu}$ of the equation for $\dot{\boldsymbol{V}}$ does not depend on $\boldsymbol{V}_{\mu}$, where $\boldsymbol{V}_{\mu}=\left(v_{1, \mu}, v_{2, \mu}, \ldots, v_{N_{p}, \mu}\right)^{\mathrm{T}}$, etc., with $1 \leqslant \mu \leqslant 3$. Therefore we can split this system once more into

$$
H_{p}=H_{p_{1}}+H_{p_{2}}+H_{p_{3}}
$$

with

$$
H_{p_{\mu}}=\frac{1}{2} \boldsymbol{V}_{\mu}^{\mathrm{T}} \boldsymbol{M}_{p} \boldsymbol{V}_{\mu} \text { for } 1 \leqslant \mu \leqslant 3
$$


For concise notation we introduce the $N_{p} \times N_{1}$ matrix $\boldsymbol{\Lambda}_{\mu}^{1}(\boldsymbol{X})$ with generic term $\boldsymbol{\Lambda}_{i, \mu}^{1}\left(\boldsymbol{x}_{a}\right)$, and the $N_{p} \times N_{p}$ diagonal matrix $\boldsymbol{\Lambda}_{\mu}^{2}(\boldsymbol{b}, \boldsymbol{X})$ with entries $\sum_{i=1}^{N_{2}} b_{i}(t) \boldsymbol{\Lambda}_{i, \mu}^{2}\left(\boldsymbol{x}_{a}\right)$, where $1 \leqslant \mu \leqslant 3,1 \leqslant a \leqslant N_{p}, 1 \leqslant i \leqslant N_{1}, 1 \leqslant j \leqslant N_{2}$. Then, for $H_{p_{1}}$ we have

$$
\begin{aligned}
\dot{\boldsymbol{X}}_{1} & =\boldsymbol{V}_{1}(t), \\
\boldsymbol{M}_{p} \dot{\boldsymbol{V}}_{2} & =-\boldsymbol{M}_{q} \boldsymbol{\Lambda}_{3}^{2}(\boldsymbol{b}(t), \boldsymbol{X}(t))^{\mathrm{T}} \boldsymbol{V}_{1}(t), \\
\boldsymbol{M}_{p} \dot{\boldsymbol{V}}_{3} & =\boldsymbol{M}_{q} \boldsymbol{\Lambda}_{2}^{2}(\boldsymbol{b}(t), \boldsymbol{X}(t))^{\mathrm{T}} \boldsymbol{V}_{1}(t), \\
\mathbb{M}_{1} \dot{\boldsymbol{e}} & =-\boldsymbol{\Lambda}_{1}^{1}(\boldsymbol{X}(t))^{\mathrm{T}} \boldsymbol{M}_{q} \boldsymbol{V}_{1}(t),
\end{aligned}
$$

for $H_{p_{2}}$ we have

$$
\begin{aligned}
\dot{\boldsymbol{X}}_{2} & =\boldsymbol{V}_{2}(t) \\
\boldsymbol{M}_{p} \dot{\boldsymbol{V}}_{1} & =\boldsymbol{M}_{q} \boldsymbol{\Lambda}_{3}^{2}(\boldsymbol{b}(t), \boldsymbol{X}(t))^{\mathrm{T}} \boldsymbol{V}_{2}(t), \\
\boldsymbol{M}_{p} \dot{\boldsymbol{V}}_{3} & =-\boldsymbol{M}_{q} \boldsymbol{\Lambda}_{1}^{2}(\boldsymbol{b}(t), \boldsymbol{X}(t))^{\mathrm{T}} \boldsymbol{V}_{2}(t), \\
\mathbb{M}_{1} \dot{\boldsymbol{e}} & =-\boldsymbol{\Lambda}_{2}^{1}(\boldsymbol{X}(t))^{\mathrm{T}} \boldsymbol{M}_{q} \boldsymbol{V}_{2}(t),
\end{aligned}
$$

and for $H_{p_{3}}$ we have

$$
\begin{aligned}
\dot{\boldsymbol{X}}_{3} & =\boldsymbol{V}_{3}(t), \\
\boldsymbol{M}_{p} \dot{\boldsymbol{V}}_{1} & =-\boldsymbol{M}_{q} \boldsymbol{\Lambda}_{2}^{2}(\boldsymbol{b}(t), \boldsymbol{X}(t))^{\mathrm{T}} \boldsymbol{V}_{3}(t), \\
\boldsymbol{M}_{p} \dot{\boldsymbol{V}}_{2} & =\boldsymbol{M}_{q} \boldsymbol{\Lambda}_{1}^{2}(\boldsymbol{b}(t), \boldsymbol{X}(t))^{\mathrm{T}} \boldsymbol{V}_{3}(t), \\
\mathbb{M}_{1} \dot{\boldsymbol{e}} & =-\boldsymbol{\Lambda}_{3}^{1}(\boldsymbol{X}(t))^{\mathrm{T}} \boldsymbol{M}_{q} \boldsymbol{V}_{3}(t) .
\end{aligned}
$$

For $H_{p_{1}}$ and initial conditions $(\boldsymbol{X}(0), \boldsymbol{V}(0), \boldsymbol{e}(0), \boldsymbol{b}(0))$ the exact solutions at time $\Delta t$ are given by the map $\varphi_{\Delta t, p_{1}}$ defined as

$$
\begin{aligned}
\boldsymbol{X}_{1}(\Delta t) & =\boldsymbol{X}_{1}(0)+\Delta t \boldsymbol{V}_{1}(0), \\
\boldsymbol{M}_{p} \boldsymbol{V}_{2}(\Delta t) & =\boldsymbol{M}_{p} \boldsymbol{V}_{2}(0)-\int_{0}^{\Delta t} \boldsymbol{M}_{q} \boldsymbol{\Lambda}_{3}^{2}(\boldsymbol{b}(0), \boldsymbol{X}(t)) \boldsymbol{V}_{1}(0) \mathrm{d} t, \\
\boldsymbol{M}_{p} \boldsymbol{V}_{3}(\Delta t) & =\boldsymbol{M}_{p} \boldsymbol{V}_{3}(0)+\int_{0}^{\Delta t} \boldsymbol{M}_{q} \boldsymbol{\Lambda}_{2}^{2}(\boldsymbol{b}(0), \boldsymbol{X}(t)) \boldsymbol{V}_{1}(0) \mathrm{d} t, \\
\mathbb{M}_{1} \boldsymbol{e}(\Delta t) & =\mathbb{M}_{1} \boldsymbol{e}(0)-\int_{0}^{\Delta t} \boldsymbol{\Lambda}_{1}^{1}(\boldsymbol{X}(t))^{\mathrm{T}} \boldsymbol{M}_{q} \boldsymbol{V}_{1}(0) \mathrm{d} t,
\end{aligned}
$$

for $H_{p_{2}}$ by the map $\varphi_{\Delta t, p_{2}}$ defined as

$$
\begin{aligned}
\boldsymbol{X}_{2}(\Delta t) & =\boldsymbol{X}_{2}(0)+\Delta t \boldsymbol{V}_{2}(0), \\
\boldsymbol{M}_{p} \boldsymbol{V}_{1}(\Delta t) & =\boldsymbol{M}_{p} \boldsymbol{V}_{1}(0)+\int_{0}^{\Delta t} \boldsymbol{M}_{q} \boldsymbol{\Lambda}_{3}^{2}(\boldsymbol{b}(0), \boldsymbol{X}(t)) \boldsymbol{V}_{2}(0) \mathrm{d} t, \\
\boldsymbol{M}_{p} \boldsymbol{V}_{3}(\Delta t) & =\boldsymbol{M}_{p} \boldsymbol{V}_{3}(0)-\int_{0}^{\Delta t} \boldsymbol{M}_{q} \boldsymbol{\Lambda}_{1}^{2}(\boldsymbol{b}(0), \boldsymbol{X}(t)) \boldsymbol{V}_{2}(0) \mathrm{d} t, \\
\mathbb{M}_{1} \boldsymbol{e}(\Delta t) & =\mathbb{M}_{1} \boldsymbol{e}(0)-\int_{0}^{\Delta t} \boldsymbol{\Lambda}_{2}^{1}(\boldsymbol{X}(t))^{\mathrm{T}} \boldsymbol{M}_{q} \boldsymbol{V}_{2}(0) \mathrm{d} t,
\end{aligned}
$$

and for $H_{p_{3}}$ by the map $\varphi_{\Delta t, p_{3}}$ defined as

$$
\boldsymbol{X}_{3}(\Delta t)=\boldsymbol{X}_{3}(0)+\Delta t \boldsymbol{V}_{3}(0)
$$




$$
\begin{aligned}
\boldsymbol{M}_{p} \boldsymbol{V}_{1}(\Delta t) & =\boldsymbol{M}_{p} \boldsymbol{V}_{1}(0)-\int_{0}^{\Delta t} \boldsymbol{M}_{q} \boldsymbol{\Lambda}_{2}^{2}(\boldsymbol{b}(0), \boldsymbol{X}(t)) \boldsymbol{V}_{3}(0) \mathrm{d} t, \\
\boldsymbol{M}_{p} \boldsymbol{V}_{2}(\Delta t) & =\boldsymbol{M}_{p} \boldsymbol{V}_{2}(0)+\int_{0}^{\Delta t} \boldsymbol{M}_{q} \boldsymbol{\Lambda}_{1}^{2}(\boldsymbol{b}(0), \boldsymbol{X}(t)) \boldsymbol{V}_{3}(0) \mathrm{d} t, \\
\mathbb{M}_{1} \boldsymbol{e}(\Delta t) & =\mathbb{M}_{1} \boldsymbol{e}(0)-\int_{0}^{\Delta t} \boldsymbol{\Lambda}_{3}^{1}(\boldsymbol{X}(t))^{\mathrm{T}} \boldsymbol{M}_{q} \boldsymbol{V}_{3}(0) \mathrm{d} t,
\end{aligned}
$$

respectively, where all components not specified are constant. The only challenge in solving these equations is the exact computation of line integrals along the trajectories (Campos Pinto et al. 2014; Squire et al. 2012; Moon et al. 2015). However, because only one component of the particle positions $\boldsymbol{x}_{a}$ is changing in each step of the splitting and, moreover, the trajectory during one sub step of the splitting is approximated by a straight line, this is not very complicated. Compared to standard particle-in-cell methods for the Vlasov-Maxwell system, the exact integration causes slightly increased computational costs. These are, however, comparable to existing charge-conserving algorithms like that of Villasenor \& Buneman (1992) or the Boris correction method (Boris 1970).

\subsection{Splitting methods}

Given initial conditions $\boldsymbol{u}(0)=(\boldsymbol{X}(0), \boldsymbol{V}(0), \boldsymbol{e}(0), \boldsymbol{b}(0))^{\mathrm{T}}$, a numerical solution of the discrete Vlasov-Maxwell equations $(4.47 a)-(4.47 d)$ at time $\Delta t$ can be obtained by composition of the exact solutions of all the subsystems. A first-order integrator can be obtained by the Lie-Trotter composition (Trotter 1959),

$$
\varphi_{\Delta t, L}=\varphi_{\Delta t, p_{3}} \circ \varphi_{\Delta t, p_{2}} \circ \varphi_{\Delta t, p_{1}} \circ \varphi_{\Delta t, B} \circ \varphi_{\Delta t, E}
$$

A second-order integrator can be obtained by the symmetric Strang composition (Strang 1968),

$$
\varphi_{\Delta t, S 2}=\varphi_{\Delta t / 2, L} \circ \varphi_{\Delta t / 2, L}^{*}
$$

where $\varphi_{\Delta t, L}^{*}$ denotes the adjoint of $\varphi_{\Delta t, L}$, defined as

$$
\varphi_{\Delta t, L}^{*}=\varphi_{-\Delta t, L}^{-1}
$$

Explicitly, the Strang splitting can be written as

$$
\begin{aligned}
\varphi_{\Delta t, S 2}= & \varphi_{\Delta t / 2, E} \circ \varphi_{\Delta t / 2, B} \circ \varphi_{\Delta t / 2, p_{1}} \circ \varphi_{\Delta t / 2, p_{2}} \circ \varphi_{\Delta t / 2, p_{3}} \\
& \circ \varphi_{\Delta t / 2, p_{3}} \circ \varphi_{\Delta t / 2, p_{2}} \circ \varphi_{\Delta t / 2, p_{1}} \circ \varphi_{\Delta t / 2, B} \circ \varphi_{\Delta t / 2, E} .
\end{aligned}
$$

Let us note that the Lie splitting $\varphi_{\Delta t, L}$ and the Strang splitting $\varphi_{\Delta t, S 2}$ are conjugate methods by the adjoint of $\varphi_{\Delta t, L}$ (McLachlan \& Quispel 2002), i.e.

$$
\varphi_{\Delta t, S 2}=\left(\varphi_{\Delta t / 2, L}^{*}\right)^{-1} \circ \varphi_{\Delta t, L} \circ \varphi_{\Delta t / 2, L}^{*}=\varphi_{-\Delta t / 2, L} \circ \varphi_{\Delta t, L} \circ \varphi_{\Delta t / 2, L}^{*}=\varphi_{\Delta t / 2, L} \circ \varphi_{\Delta t / 2, L}^{*}
$$

The last equality holds by the group property of the flow, but is only valid when the exact solution of each subsystem is used in the composition (and not some general symplectic or Poisson integrator). This implies that the Lie splitting shares many properties with the Strang splitting which are not found in general first-order methods. 
Another second-order integrator with a smaller error constant than $\varphi_{\Delta t, S 2}$ can be obtained by the following composition,

$$
\varphi_{\Delta t, L 2}=\varphi_{\alpha \Delta t, L} \circ \varphi_{(1 / 2-\alpha) \Delta t, L}^{*} \circ \varphi_{(1 / 2-\alpha) \Delta t, L} \circ \varphi_{\alpha \Delta t, L}^{*}
$$

Here, $\alpha$ is a free parameter which can be used to reduce the error constant. A particularly small error is obtained for $\alpha=0.1932$ (McLachlan 1995). Fourth-order time integrators can easily be obtained from a second-order integrator like $\varphi_{\Delta t, S}$ by the following composition (Suzuki 1990; Yoshida 1990),

$$
\varphi_{\Delta t, S 4}=\varphi_{\gamma_{1} \Delta t, S 2} \circ \varphi_{\gamma_{2} \Delta t, S 2} \circ \varphi_{\gamma_{1} \Delta t, S 2}
$$

with

$$
\gamma_{1}=\frac{1}{2-2^{1 / 3}}, \quad \gamma_{2}=-\frac{2^{1 / 3}}{2-2^{1 / 3}} .
$$

Alternatively, we can compose the first-order integrator $\varphi_{\Delta t, L}$ together with its adjoint $\varphi_{\Delta t, L}^{*}$ as follows (McLachlan 1995),

$$
\varphi_{\Delta t, L 4}=\varphi_{a_{5} \Delta t, L} \circ \varphi_{b_{5} \Delta t, L}^{*} \circ \ldots \circ \varphi_{a_{2} \Delta t, L} \circ \varphi_{b_{2} \Delta t, L}^{*} \circ \varphi_{a_{1} \Delta t, L} \circ \varphi_{b_{1} \Delta t, L}^{*}
$$

with

$$
\begin{gathered}
a_{1}=b_{5}=\frac{146+5 \sqrt{19}}{540}, \quad a_{2}=b_{4}=\frac{-2+10 \sqrt{19}}{135}, \quad a_{3}=b_{3}=\frac{1}{5} \\
a_{4}=b_{2}=\frac{-23-20 \sqrt{19}}{270}, \quad a_{5}=b_{1}=\frac{14-\sqrt{19}}{108}
\end{gathered}
$$

For higher-order composition methods see e.g. Hairer et al. (2006) and McLachlan \& Quispel (2002) and references therein.

\subsection{Backward error analysis}

In the following, we want to compute the modified Hamiltonian for the Lie-Trotter composition (5.17). For a splitting in two terms, $H=H_{A}+H_{B}$, the Lie-Trotter composition can be written as

$$
\varphi_{\Delta t}=\varphi_{\Delta t, B} \circ \varphi_{\Delta t, A}=\exp \left(\Delta t \boldsymbol{X}_{A}\right) \exp \left(\Delta t \boldsymbol{X}_{B}\right)=\exp (\Delta t \tilde{\boldsymbol{X}})
$$

where $\boldsymbol{X}_{A}$ and $\boldsymbol{X}_{B}$ are the Hamiltonian vector fields corresponding to $H_{A}$ and $H_{B}$, respectively, and $\tilde{\boldsymbol{X}}$ is the modified vector field corresponding to the modified Hamiltonian $\tilde{H}$. Following Hairer et al. (2006, Chapter IX), the modified Hamiltonian $\tilde{H}$ is given by

$$
\tilde{H}=H+\Delta t \tilde{H}_{1}+\Delta t^{2} \tilde{H}_{2}+O\left(\Delta t^{3}\right),
$$

with

$$
\begin{gathered}
\tilde{H}_{1}=\frac{1}{2}\left\{H_{A}, H_{B}\right\}, \\
\tilde{H}_{2}=\frac{1}{12}\left[\left\{\left\{H_{A}, H_{B}\right\}, H_{B}\right\}+\left\{\left\{H_{B}, H_{A}\right\}, H_{A}\right\}\right] .
\end{gathered}
$$

In order to compute the modified Hamiltonian for splittings with more than two terms, we have to apply the Baker-Campbell-Hausdorff formula recursively. Denoting by $\boldsymbol{X}_{E}$ 
the Hamiltonian vector field corresponding to $H_{E}$, that is $\boldsymbol{X}_{E}=\rrbracket\left(\cdot, D H_{E}\right)$, and similar for $\boldsymbol{X}_{B}$ and $\boldsymbol{X}_{p_{i}}$, the Lie-Trotter splitting (5.17) can be expressed in terms of these Hamiltonian vector fields as

$$
\varphi_{\Delta t, L}=\exp \left(\Delta t \boldsymbol{X}_{E}\right) \exp \left(\Delta t \boldsymbol{X}_{B}\right) \exp \left(\Delta t \boldsymbol{X}_{p_{1}}\right) \exp \left(\Delta t \boldsymbol{X}_{p_{2}}\right) \exp \left(\Delta t \boldsymbol{X}_{p_{3}}\right)
$$

Let us split $\varphi_{\Delta t}=\exp (\Delta t \tilde{\boldsymbol{X}})$ into

$$
\left.\begin{array}{rl}
\exp \left(\Delta t \boldsymbol{X}_{E}\right) \exp \left(\Delta t \boldsymbol{X}_{B}\right) & =\exp \left(\Delta t \tilde{\boldsymbol{X}}_{E B}\right), \\
\exp \left(\Delta t \boldsymbol{X}_{p_{1}}\right) \exp \left(\Delta t \boldsymbol{X}_{p_{2}}\right) & =\exp \left(\Delta t \tilde{\boldsymbol{X}}_{p_{1,2}}\right), \\
\exp \left(\Delta t \tilde{\boldsymbol{X}}_{p_{1,2}}\right) \exp \left(h \boldsymbol{X}_{p_{3}}\right) & =\exp \left(\Delta t \tilde{\boldsymbol{X}}_{p_{1,2,3}}\right), \\
\exp \left(\Delta t \tilde{\boldsymbol{X}}_{E B}\right) \exp \left(\Delta t \tilde{\boldsymbol{X}}_{p_{1,2,3}}\right) & =\exp (\Delta t \tilde{\boldsymbol{X}}),
\end{array}\right\}
$$

where the corresponding Hamiltonians are given by

$$
\left.\begin{array}{rl}
\tilde{H}_{E B} & =H_{E}+H_{B}+\frac{\Delta t}{2}\left\{H_{E}, H_{B}\right\}+O\left(\Delta t^{2}\right), \\
\tilde{H}_{p_{1,2}} & =H_{p_{1}}+H_{p_{2}}+\frac{\Delta t}{2}\left\{H_{p_{1}}, H_{p_{2}}\right\}+O\left(\Delta t^{2}\right), \\
\tilde{H}_{p_{1,2,3}} & =\tilde{H}_{p_{1,2}}+H_{p_{3}}+\frac{\Delta t}{2}\left\{\tilde{H}_{p_{1,2}}, H_{p_{3}}\right\}+O\left(\Delta t^{2}\right) \\
& =H_{p_{1}}+H_{p_{2}}+H_{p_{3}}+\frac{\Delta t}{2}\left\{H_{p_{1}}, H_{p_{2}}\right\}+\frac{\Delta t}{2}\left\{H_{p_{1}}+H_{p_{2}}, H_{p_{3}}\right\}+O\left(\Delta t^{2}\right) .
\end{array}\right\}
$$

The Hamiltonian $\tilde{H}$ corresponding to $\tilde{X}$ is given by

$$
\tilde{H}=H_{E}+H_{B}+H_{p_{1}}+H_{p_{2}}+H_{p_{3}}+\Delta t \tilde{H}_{1}+O\left(\Delta t^{2}\right),
$$

with the first-order correction $\tilde{H}_{1}$ obtained as

$$
\tilde{H}_{1}=\frac{1}{2}\left(\left\{H_{E}, H_{B}\right\}+\left\{H_{p_{1}}, H_{p_{2}}\right\}+\left\{H_{p_{1}}+H_{p_{2}}, H_{p_{3}}\right\}+\left\{H_{E}+H_{B}, H_{p}\right\}\right) .
$$

The various Poisson brackets are computed as follows,

$$
\left.\begin{array}{rl}
\left\{H_{E}, H_{B}\right\} & =\boldsymbol{e}^{\mathrm{T}} \mathbb{C}^{\mathrm{T}} \boldsymbol{b}, \\
\left\{H_{E}, H_{p}\right\} & =-\boldsymbol{e}^{\mathrm{T}} \boldsymbol{\Lambda}^{1}(\boldsymbol{X})^{\mathrm{T}} \mathbb{M}_{q} \boldsymbol{V}, \\
\left\{H_{B}, H_{p}\right\} & =0, \\
\left\{H_{p_{1}}, H_{p_{2}}\right\} & =\boldsymbol{V}_{1} \boldsymbol{M}_{q} \mathbb{B}_{3}(\boldsymbol{b}, \boldsymbol{X})^{\mathrm{T}} \boldsymbol{V}_{2}, \\
\left\{H_{p_{2}}, H_{p_{3}}\right\} & =\boldsymbol{V}_{2} \boldsymbol{M}_{q} \mathbb{B}_{1}(\boldsymbol{b}, \boldsymbol{X})^{\mathrm{T}} \boldsymbol{V}_{3}, \\
\left\{H_{p_{3}}, H_{p_{1}}\right\} & =\boldsymbol{V}_{3} \boldsymbol{M}_{q} \mathbb{B}_{2}(\boldsymbol{b}, \boldsymbol{X})^{\mathrm{T}} \boldsymbol{V}_{1},
\end{array}\right\}
$$

where $\mathbb{B}_{\mu}(\boldsymbol{X}, \boldsymbol{b})$ denotes $N_{p} \times N_{p}$ diagonal matrix with elements $B_{h, \mu}\left(\boldsymbol{x}_{a}\right)$. The LieTrotter integrator (5.17) preserves the modified energy $\tilde{H}=H+\Delta t \tilde{H}_{1}$ to $\mathcal{O}\left(\Delta t^{2}\right)$, while the original energy $H$ is preserved only to $\mathcal{O}(\Delta t)$.

\section{Example: Vlasov-Maxwell in $1 \mathrm{~d} 2 \mathrm{v}$}

Starting from the Vlasov equation for a particle species $s$, charge $q_{s}$, and mass $m_{s}$ given in (2.1), we reduce by assuming a single species and

$$
\boldsymbol{x}=(x, 0,0) \text { and } \boldsymbol{v}=\left(v_{1}, v_{2}, 0\right)
$$


as well as

$$
\boldsymbol{E}(x, t)=\left(E_{1}, E_{2}, 0\right), \quad \boldsymbol{B}(x, t)=\left(0,0, B_{3}\right), \quad f(\boldsymbol{x}, \boldsymbol{v}, t)=f\left(x, v_{1}, v_{2}, t\right), \quad(6.2 a-c)
$$

so that the Vlasov equation takes the form

$$
\frac{\partial f(x, \boldsymbol{v}, t)}{\partial t}+v_{1} \frac{\partial f(x, \boldsymbol{v}, t)}{\partial x}+\frac{q_{s}}{m_{s}}\left[\boldsymbol{E}(x, t)+B_{3}(x, t)\left(\begin{array}{c}
v_{2} \\
-v_{1}
\end{array}\right)\right] \cdot \nabla_{v} f(x, v, t)=0,
$$

while Maxwell's equations become

$$
\begin{aligned}
\frac{\partial E_{1}(x, t)}{\partial t} & =-J_{1}(x) \\
\frac{\partial E_{2}(x, t)}{\partial t} & =-\frac{\partial B(x, t)}{\partial x}-J_{2}(x) \\
\frac{\partial B(x, t)}{\partial t} & =-\frac{\partial E_{2}(x, t)}{\partial x} \\
\frac{\partial E_{1}(x, t)}{\partial x} & =\rho+\rho_{B}
\end{aligned}
$$

with sources given by

$$
\rho=q_{s} \int f \mathrm{~d} \boldsymbol{v}, \quad J_{1}=q_{s} \int f v_{1} \mathrm{~d} \boldsymbol{v}, \quad J_{2}=q_{s} \int f v_{2} \mathrm{~d} \boldsymbol{v} .
$$

Note that $\operatorname{div} \boldsymbol{B}=0$ is manifest.

\subsection{Non-canonical Hamiltonian structure}

Under the assumptions of (6.1) and (6.2), the bracket of (2.8) reduces to

$$
\begin{aligned}
\{\mathcal{F}, \mathcal{G}\}= & \frac{1}{m} \int f\left[\frac{\delta \mathcal{F}}{\delta f}, \frac{\delta \mathcal{G}}{\delta f}\right] \mathrm{d} x \mathrm{~d} v_{1} \mathrm{~d} v_{2} \\
& +\frac{q}{m} \int f\left(\frac{\partial}{\partial v_{1}} \frac{\delta \mathcal{F}}{\delta f} \frac{\delta \mathcal{G}}{\delta E_{1}}+\frac{\partial}{\partial v_{2}} \frac{\delta \mathcal{F}}{\delta f} \frac{\delta \mathcal{G}}{\delta E_{2}}-\frac{\partial}{\partial v_{1}} \frac{\delta \mathcal{G}}{\delta f} \frac{\delta \mathcal{F}}{\delta E_{1}}-\frac{\partial}{\partial v_{2}} \frac{\delta \mathcal{G}}{\delta f} \frac{\delta \mathcal{F}}{\delta E_{2}}\right) \\
& \times \mathrm{d} x \mathrm{~d} v_{1} \mathrm{~d} v_{2} \\
& +\frac{q}{m^{2}} \int f B\left(\frac{\partial}{\partial v_{1}} \frac{\delta \mathcal{F}}{\delta f} \frac{\partial}{\partial v_{2}} \frac{\delta \mathcal{G}}{\delta f}-\frac{\partial}{\partial v_{2}} \frac{\delta \mathcal{F}}{\delta f} \frac{\partial}{\partial v_{1}} \frac{\delta \mathcal{G}}{\delta f}\right) \mathrm{d} x \mathrm{~d} v_{1} \mathrm{~d} v_{2} \\
& +\int\left(\frac{\delta \mathcal{G}}{\delta E_{2}} \frac{\partial}{\partial x} \frac{\delta \mathcal{F}}{\delta B}-\frac{\delta \mathcal{F}}{\delta E_{2}} \frac{\partial}{\partial x} \frac{\delta \mathcal{G}}{\delta B}\right) \mathrm{d} x,
\end{aligned}
$$

where now

$$
[f, g]:=\frac{\partial f}{\partial x} \frac{\partial g}{\partial v_{1}}-\frac{\partial f}{\partial v_{1}} \frac{\partial g}{\partial x} .
$$

The Hamiltonian (2.11) becomes

$$
\mathcal{H}=\frac{m}{2} \int f\left(x, v_{1}, v_{2}\right)\left(v_{1}^{2}+v_{2}^{2}\right) \mathrm{d} x \mathrm{~d} v_{1} \mathrm{~d} v_{2}+\frac{1}{2} \int\left(E_{1}^{2}+E_{2}^{2}+B^{2}\right) \mathrm{d} x .
$$

The bracket of (6.9) with Hamiltonian (6.11), generates (6.4), (6.5) and (6.6), with the $\boldsymbol{J}$ of (6.8). 


\subsection{Reduced Jacobi identity}

The bracket of (6.9) can be shown to satisfy the Jacobi identity by direct calculations. However since (2.8) satisfies the Jacobi identity for all functionals, it must also satisfy it for all reduced functionals. Here we have closure, i.e. if $\mathcal{F}$ and $\mathcal{G}$ are reduced functionals, then $\{\mathcal{F}, \mathcal{G}\}$ is a reduced functional, where the bracket is the full bracket of (2.8).

More specifically, to understand this closure, observe that the reduced functionals have the form

$$
\begin{aligned}
\mathcal{F}\left[E_{1}, E_{2}, B, f\right] & =\int \mathcal{F}(\boldsymbol{x}, \boldsymbol{E}, \boldsymbol{B}, f, D \boldsymbol{E}, D \boldsymbol{B}, D f, \ldots) \mathrm{d} \boldsymbol{x} \mathrm{d} \boldsymbol{v} \\
& =\int \mathcal{F}\left(x, E_{1}, E_{2}, B, f, D E_{1}, D E_{2}, D B, D f, \ldots\right) \mathrm{d} \boldsymbol{x} \mathrm{d} \boldsymbol{v} \\
& =\int \overline{\mathcal{F}}\left(x, E_{1}, E_{2}, B, f, D E_{1}, D E_{2}, D B, D f, \ldots\right) \mathrm{d} x \mathrm{~d} v_{1} \mathrm{~d} v_{2},
\end{aligned}
$$

where in (6.12) we assumed (6.1) and (6.2). In the second equality of (6.12) the integrations over $x_{2}, x_{3}$ and $v_{3}$ are easily performed because the integrand is independent of these variables or it has been performed with an explicit dependence on $x_{2}, x_{3}$ and $v_{3}$ that makes the integrals converge. Any constant factors resulting from the integrations are absorbed into the definition of $\overline{\mathcal{F}}$. The closure condition on $\{\mathcal{F}, \mathcal{G}\}$ amounts to the statement that given any two functionals $\mathcal{F}, \mathcal{G}$ of the form of (6.12), their bracket is again a reduced functional of this form. This follows from the fact that the bracket of two such functionals reduces (2.8) to (6.9), which of course is reduced.

That not all reductions of functionals have closure can be seen by considering ones of the form $\mathcal{F}[\boldsymbol{E}, f]$, i.e. ones for which dependence on $\boldsymbol{B}$ is absent. The bracket of two functions of this form gives the bracket of (2.8) with the absence of the last term. Clearly this bracket depends on $\boldsymbol{B}$ and thus there is no closure. A consequence of this is that the bracket (2.8) with the absence of the last term does not satisfy the Jacobi identity. We note, however, that adding a projector can remedy this, as was shown in Chandre et al. (2013).

\subsection{Discrete deRham complex in one dimension}

Here, we consider the components of the electromagnetic fields separately and we have that $E_{1}$ is a 1 -form, $E_{2}$ is a 0 -form and $B_{3}$ is again a 1 -form. We denote the semi-discrete fields by $D_{h}, E_{h}$ and $B_{h}$ respectively, and write

$$
\left.\begin{array}{l}
D_{h}(x, t)=\sum_{i=1}^{N_{1}} d_{i}(t) \Lambda_{i}^{1}(x), \\
E_{h}(x, t)=\sum_{i=1}^{N_{0}} e_{i}(t) \Lambda_{i}^{0}(x), \\
B_{h}(x, t)=\sum_{i=1}^{N_{1}} b_{i}(t) \Lambda_{i}^{1}(x) .
\end{array}\right\}
$$

Next we introduce an equidistant grid in $x$ and denote the spline of degree $p$ with support starting at $x_{i}$ by $N_{i}^{p}$. We can express the derivative of $N_{i}^{p}$ as follows

$$
\frac{\mathrm{d}}{\mathrm{d} x} N_{i}^{p}(x)=\frac{1}{\Delta x}\left(N_{i}^{p-1}(x)-N_{i+1}^{p-1}(x)\right) .
$$


In the finite element field solver, we represent $E_{h}$ by an expansion in splines of degree $p$ and $D_{h}, B_{h}$ by an expansion in splines of degree $p-1$, that is

$$
\left.\begin{array}{l}
D_{h}(x, t)=\sum_{i=1}^{N_{1}} d_{i}(t) N_{i}^{p-1}(x), \\
E_{h}(x, t)=\sum_{i=1}^{N_{0}} e_{i}(t) N_{i}^{p}(x), \\
B_{h}(x, t)=\sum_{i=1}^{N_{1}} b_{i}(t) N_{i}^{p-1}(x) .
\end{array}\right\}
$$

\subsection{Discrete Poisson bracket}

The discrete Poisson bracket for this reduced system reads

$$
\begin{aligned}
\{F, G\}\left[\boldsymbol{X}, \boldsymbol{V}_{1}, \boldsymbol{V}_{2}, \boldsymbol{d}, \boldsymbol{e}, \boldsymbol{b}\right] \\
=\frac{\partial F}{\partial \boldsymbol{X}_{1}} \mathbb{M}_{p}^{-1} \frac{\partial G}{\partial \boldsymbol{V}_{1}}-\frac{\partial G}{\partial \boldsymbol{X}_{1}} \mathbb{M}_{p}^{-1} \frac{\partial F}{\partial \boldsymbol{V}_{1}} \\
+\left(\frac{\partial F}{\partial \boldsymbol{V}_{1}}\right)^{\mathrm{T}} \mathbb{M}_{p}^{-1} \mathbb{M}_{q} \boldsymbol{\Lambda}^{1}(\boldsymbol{X})^{\mathrm{T}} \mathbb{M}_{1}^{-1}\left(\frac{\partial G}{\partial \boldsymbol{d}}\right)-\left(\frac{\partial F}{\partial \boldsymbol{d}}\right)^{\mathrm{T}} \mathbb{M}_{1}^{-1} \boldsymbol{\Lambda}^{1}(\boldsymbol{X}) \mathbb{M}_{q} \mathbb{M}_{p}^{-1}\left(\frac{\partial G}{\partial \boldsymbol{V}_{1}}\right) \\
+\left(\frac{\partial F}{\partial \boldsymbol{V}_{2}}\right)^{\mathrm{T}} \mathbb{M}_{p}^{-1} \mathbb{M}_{q} \boldsymbol{\Lambda}^{0}(\boldsymbol{X})^{\mathrm{T}} \mathbb{M}_{0}^{-1}\left(\frac{\partial G}{\partial \boldsymbol{e}}\right)-\left(\frac{\partial F}{\partial \boldsymbol{e}}\right)^{\mathrm{T}} \mathbb{M}_{0}^{-1} \Lambda^{0}(\boldsymbol{X}) \mathbb{M}_{q} \mathbb{M}_{p}^{-1}\left(\frac{\partial G}{\partial \boldsymbol{V}_{2}}\right) \\
+\left(\frac{\partial F}{\partial \boldsymbol{V}_{1}}\right)^{\mathrm{T}} \mathbb{M}_{p}^{-1} \mathbb{M}_{q} \mathbb{B}(\boldsymbol{X}, \boldsymbol{b}) \mathbb{M}_{p}^{-1}\left(\frac{\partial G}{\partial \boldsymbol{V}_{2}}\right)-\left(\frac{\partial F}{\partial \boldsymbol{V}_{2}}\right)^{\mathrm{T}} \mathbb{M}_{p}^{-1} \mathbb{B}(\boldsymbol{X}, \boldsymbol{b}) \mathbb{M}_{q} \mathbb{M}_{p}^{-1}\left(\frac{\partial G}{\partial \boldsymbol{V}_{1}}\right) \\
+\left(\frac{\partial F}{\partial \boldsymbol{e}}\right)^{\mathrm{T}} \mathbb{M}_{0}^{-1} \mathbb{C}^{\mathrm{T}}\left(\frac{\partial G}{\partial \boldsymbol{b}}\right)-\left(\frac{\partial F}{\partial \boldsymbol{b}}\right)^{\mathrm{T}} \mathbb{C M}_{0}^{-1}\left(\frac{\partial G}{\partial \boldsymbol{e}}\right) .
\end{aligned}
$$

Here, we denote by $\mathbb{M}_{p}=\boldsymbol{M}_{p}$ and $\mathbb{M}_{q}=\boldsymbol{M}_{q}$ the $N_{p} \times N_{p}$ diagonal matrices holding the particle masses and charges, respectively. We denote by $\boldsymbol{\Lambda}^{0}(\boldsymbol{X})$ the $N_{p} \times N_{0}$ matrix with generic term $\Lambda_{i}^{0}\left(x_{a}\right)$, where $1 \leqslant a \leqslant N_{p}$ and $1 \leqslant i \leqslant N_{0}$, and by $\Lambda^{1}(X)$ the $N_{p} \times N_{1}$ matrix with generic term $\Lambda_{i}^{1}\left(x_{a}\right)$, where $1 \leqslant a \leqslant N_{p}$ and $1 \leqslant i \leqslant N_{1}$. Further, $\mathbb{B}(\boldsymbol{X}, \boldsymbol{b})$ denotes the $N_{p} \times N_{p}$ diagonal matrix with entries

$$
B_{h}\left(x_{a}, t\right)=\sum_{i=1}^{N_{1}} b_{i}(t) \Lambda_{i}^{1}\left(x_{a}\right)
$$

The reduced bracket can be shown to satisfy the Jacobi identity by direct proof in full analogy to the proof for the full bracket shown in $\$ 4.4$. However, one can also follow along the lines of $\S 6.2$ in order to arrive at the same result.

\subsection{Discrete Hamiltonian and equations of motion}

The discrete Hamiltonian is given in terms of the reduced set of degrees of freedom $\boldsymbol{u}=\left(\boldsymbol{X}, \boldsymbol{V}_{1}, \boldsymbol{V}_{2}, \boldsymbol{d}, \boldsymbol{e}, \boldsymbol{b}\right)$ by

$$
H=\frac{1}{2} \boldsymbol{V}_{1}^{\mathrm{T}} \mathbb{M}_{p} \boldsymbol{V}_{1}+\frac{1}{2} \boldsymbol{V}_{2}^{\mathrm{T}} \mathbb{M}_{p} \boldsymbol{V}_{2}+\frac{1}{2} \boldsymbol{d}^{\mathrm{T}} \mathbb{M}_{1} \boldsymbol{d}+\frac{1}{2} \boldsymbol{e}^{\mathrm{T}} \mathbb{M}_{0} \boldsymbol{e}+\frac{1}{2} \boldsymbol{b}^{\mathrm{T}} \mathbb{M}_{1} \boldsymbol{b}
$$


and the equations of motion are obtained as

$$
\begin{aligned}
\dot{\boldsymbol{X}} & =\boldsymbol{V}_{1}, \\
\dot{\boldsymbol{V}}_{1} & =\mathbb{M}_{p}^{-1} \mathbb{M}_{q}\left(\wedge^{1}(\boldsymbol{X}) \boldsymbol{d}+\mathbb{B}(\boldsymbol{X}, \boldsymbol{b}) \boldsymbol{V}_{2}\right), \\
\dot{\boldsymbol{V}}_{2} & =\mathbb{M}_{p}^{-1} \mathbb{M}_{q}\left(\boldsymbol{\Lambda}^{0}(\boldsymbol{X}) \boldsymbol{e}-\mathbb{B}(\boldsymbol{X}, \boldsymbol{b}) \boldsymbol{V}_{1}\right), \\
\dot{\boldsymbol{d}} & =-\mathbb{M}_{1}^{-1} \bigwedge^{1}(\boldsymbol{X})^{\mathrm{T}} \mathbb{M}_{q} \boldsymbol{V}_{1}, \\
\dot{\boldsymbol{e}} & =\mathbb{M}_{0}^{-1}\left(\mathbb{C}^{\mathrm{T}} \mathbb{M}_{1} \boldsymbol{b}(t)-\mathbb{\Lambda}^{0}(\boldsymbol{X})^{\mathrm{T}} \mathbb{M}_{q} \boldsymbol{V}_{2}\right), \\
\dot{\boldsymbol{b}} & =-\mathbb{C} \boldsymbol{e}(t),
\end{aligned}
$$

which is seen to be in direct correspondence with (6.3)-(6.7).

\subsection{Hamiltonian splitting}

The solution of the discrete equations of motion for $H_{D}+H_{E}$ at time $\Delta t$ is

$$
\left.\begin{array}{rl}
\mathbb{M}_{p} \boldsymbol{V}_{1}(\Delta t) & =\mathbb{M}_{p} \boldsymbol{V}_{1}(0)+\Delta t \mathbb{M}_{q} \AA^{1}(\boldsymbol{X}(0)) \boldsymbol{d}(0), \\
\mathbb{M}_{p} \boldsymbol{V}_{2}(\Delta t) & =\mathbb{M}_{p} \boldsymbol{V}_{2}(0)+\Delta t \mathbb{M}_{q} \AA^{0}(\boldsymbol{X}(0)) \boldsymbol{e}(0), \\
\boldsymbol{b}(\Delta t) & =\boldsymbol{b}(0)-\Delta t \mathbb{C} \boldsymbol{e}(0) .
\end{array}\right\}
$$

The solution of the discrete equations of motion for $H_{B}$ is

$$
\mathbb{M}_{0} \boldsymbol{e}(\Delta t)=\mathbb{M}_{0} \boldsymbol{e}(0)+\Delta t \mathbb{C}^{\mathrm{T}} \mathbb{M}_{1} \boldsymbol{b}(0) .
$$

The solution of the discrete equations of motion for $H_{p_{1}}$ is

$$
\left.\begin{array}{rl}
\boldsymbol{X}(\Delta t) & =\boldsymbol{X}_{1}(0)+\Delta t \boldsymbol{V}_{1}(0), \\
\mathbb{M}_{p} \boldsymbol{V}_{2}(\Delta t) & =\mathbb{M}_{p} \boldsymbol{V}_{2}(0)-\int_{0}^{\Delta t} \mathbb{M}_{q} \mathbb{B}(\boldsymbol{X}(t), \boldsymbol{b}(0)) \boldsymbol{V}_{1}(0) \mathrm{d} t, \\
\mathbb{M}_{1} \boldsymbol{d}(\Delta t) & =\mathbb{M}_{1} \boldsymbol{d}(0)-\int_{0}^{\Delta t} \Delta^{1}(\boldsymbol{X}(t))^{\mathrm{T}} \mathbb{M}_{q} \boldsymbol{V}_{1}(0) \mathrm{d} t,
\end{array}\right\}
$$

and for $H_{p_{2}}$ it is

$$
\left.\begin{array}{c}
\mathbb{M}_{p} \boldsymbol{V}_{1}(\Delta t)=\mathbb{M}_{p} \boldsymbol{V}_{1}(0)+\int_{0}^{\Delta t} \mathbb{M}_{q} \mathbb{B}(\boldsymbol{X}(0), \boldsymbol{b}(0)) \boldsymbol{V}_{2}(0) \mathrm{d} t, \\
\mathbb{M}_{0} \boldsymbol{e}(\Delta t)=\mathbb{M}_{0} \boldsymbol{e}(0)-\int_{0}^{\Delta t} \Lambda^{1}(\boldsymbol{X}(0))^{\mathrm{T}} \mathbb{M}_{q} \boldsymbol{V}_{2}(0) \mathrm{d} t,
\end{array}\right\}
$$

respectively.

\section{Numerical experiments}

We have implemented the Hamiltonian splitting scheme as well as the Boris-Yee scheme from appendix A as part of the SeLaLib library (http://selalib.gforge.inria.fr/). In this section, we present results for various test cases in $1 \mathrm{~d} 2 \mathrm{v}$, comparing the conservation properties of the total energy and of the Casimirs for the two schemes. We simulate the electron distribution function in a neutralizing ion background. In all experiments, we have used splines of order three for the 0 -forms. The particle loading was done using Sobol numbers and antithetic sampling (symmetric around the middle of the domain in $x$ and around the mean value of the Gaussian from which we are sampling in each velocity dimension). We sample uniformly in $x$ and from the Gaussians of the initial distribution in each velocity dimension. 


\subsection{Weibel instability}

We consider the Weibel instability studied in Weibel (1959) in the form simulated in Crouseilles et al. (2015). We study a reduced $1 \mathrm{~d} 2 \mathrm{v}$ model with a perturbation along $x_{1}$, a magnetic field along $x_{3}$ and electric fields along the $x_{1}$ and $x_{2}$ directions. Moreover, we assume that the distribution function is independent of $v_{3}$. The initial distribution and fields are of the form

$$
\begin{gathered}
f(x, \boldsymbol{v}, t=0)=\frac{1}{2 \pi \sigma_{1} \sigma_{2}} \exp \left(-\frac{1}{2}\left(\frac{v_{1}^{2}}{\sigma_{1}^{2}}+\frac{v_{2}^{2}}{\sigma_{2}^{2}}\right)\right)(1+\alpha \cos (k x)), \quad x \in[0,2 \pi / k), \\
B_{3}(x, t=0)=\beta \cos (k x), \\
E_{2}(x, t=0)=0,
\end{gathered}
$$

and $E_{1}(x, t=0)$ is computed from Poisson's equation. In our simulations, we use the following choice of parameters, $\sigma_{1}=0.02 / \sqrt{2}, \sigma_{2}=\sqrt{12} \sigma_{1}, k=1.25, \alpha=0$ and $\beta=$ $-10^{-4}$. Note that these are the same parameters as in Crouseilles et al. (2015) except for the fact that we sample from the Maxwellian without perturbation in $x_{1}$.

The dispersion relation from Weibel (1959) applied to our model reads

$$
D(\omega, k)=\omega^{2}-k^{2}+\left(\frac{\sigma_{2}}{\sigma_{1}}\right)^{2}-1-\left(\frac{\sigma_{2}}{\sigma_{1}}\right)^{2} \phi\left(\frac{\omega}{\sigma_{1} k}\right) \frac{\omega}{\sigma_{1} k}
$$

where $\phi(z)=\exp \left(-1 / 2 z^{2}\right) \int_{-\mathrm{i} \infty}^{z} \exp \left(1 / 2 \xi^{2}\right) \mathrm{d} \xi$. For our parameter choice, this gives a growth rate of 0.02784 . In figure 1 , we show the electric and magnetic energies together with the analytic growth rate. We see that the growth rate is verified in the numerical solution. This simulation was performed with 100000 particles, 32 grid points, splines of degree 3 and 2 and $\Delta t=0.05$. Note that we have chosen a very large number of particles in order to obtain a solution of very high quality. In practice, the Weibel instability can also be simulated with much fewer particles (cf. § 7.5).

In table 1, we show the conservation properties of our splitting with various orders of the splitting (cf. § 5.2) and compare them also to the Boris-Yee scheme. The other numerical parameters are kept as before.

We can see that Gauss' law is satisfied in each time step for the Hamiltonian splitting. This is a Casimir (cf. §2.2) and therefore naturally conserved by the Hamiltonian splitting. On the other hand, this is not the case for the Boris-Yee scheme.

We can also see that the energy error improves with the order of the splitting; however, the Hamiltonian splitting method as well as the Boris-Yee scheme are not energy conserving. The time evolution of the total energy error is depicted in figure 2 for the various methods.

\subsection{Streaming Weibel instability}

As a second test case, we consider the streaming Weibel instability. We study the same reduced model as in the previous section, but following Califano, Pegoraro \& Bulanov (1997), Cheng et al. (2014c) the initial distribution and fields are prescribed as 


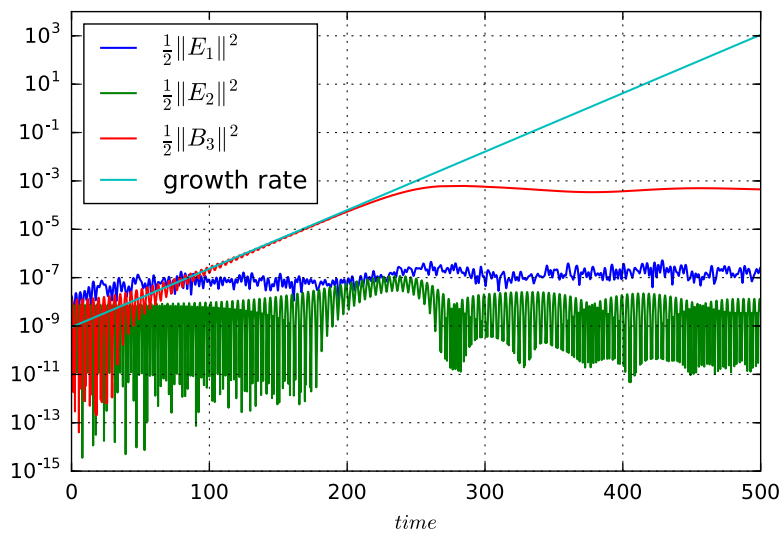

FIGURE 1. Weibel instability: the two electric and the magnetic energies together with the analytic growth rate.

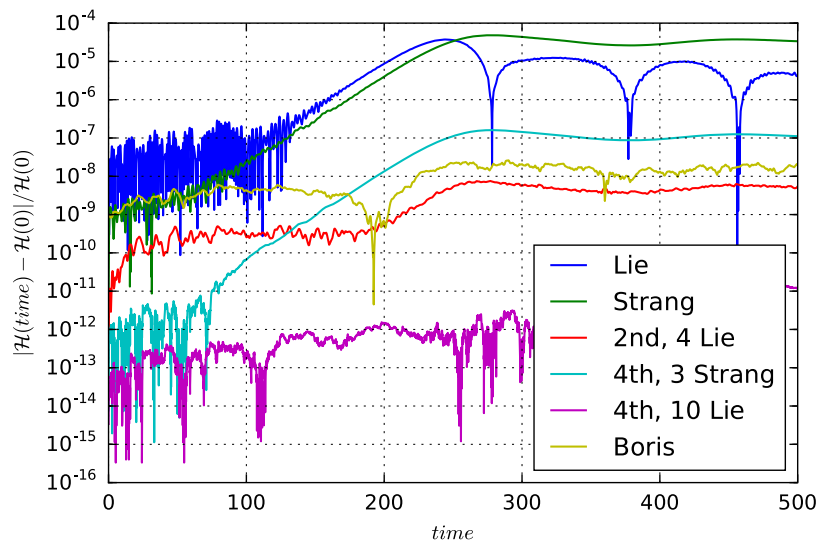

FIGURE 2. Weibel instability: difference of total energy and its initial value as a function of time for various integrators.

$\begin{array}{lcc}\text { Propagator } & \text { Total energy } & \text { Gauss' law } \\ \text { Lie } & 4.9 \times 10^{-7} & 1.4 \times 10^{-15} \\ \text { Strang } & 6.3 \times 10^{-7} & 1.2 \times 10^{-15} \\ \text { 2nd, 4 Lie } & 9.8 \times 10^{-11} & 1.4 \times 10^{-15} \\ \text { 4th, 3 Strang } & 2.1 \times 10^{-9} & 1.2 \times 10^{-15} \\ \text { 4th, 10 Lie } & 2.1 \times 10^{-13} & 1.0 \times 10^{-15} \\ \text { Boris } & 3.4 \times 10^{-10} & 4.4 \times 10^{-5}\end{array}$

TABLE 1. Weibel instability: maximum error in the total energy and Gauss' law until time 500 for simulation with various integrators: Lie-Trotter splitting from (5.17) (Lie), Strang splitting from (5.18) (Strang), second-order splitting with 4 Lie parts defined in (5.22) (2nd, $4 \mathrm{Lie}$ ), fourth-order splitting with 3 Strang parts defined in (5.23) (4th, 3 Strang) and 10 Lie parts defined in (5.25) (4th, $10 \mathrm{Lie})$. 


$\begin{array}{lcc}\text { Propagator } & \text { Total energy } & \text { Gauss' law } \\ \text { Lie } & 6.4 \times 10^{-5} & 3.1 \times 10^{-16} \\ \text { Strang } & 1.4 \times 10^{-6} & 3.7 \times 10^{-16} \\ \text { 2nd, 4 Lie } & 1.5 \times 10^{-8} & 4.4 \times 10^{-16} \\ \text { 4th, 3 Strang } & 1.7 \times 10^{-10} & 4.7 \times 10^{-16} \\ \text { 4th, 10 Lie } & 5.7 \times 10^{-13} & 5.4 \times 10^{-16} \\ \text { Boris } & 1.1 \times 10^{-7} & 5.1 \times 10^{-4}\end{array}$

TABLE 2. Streaming Weibel instability: maximum error in the total energy and Gauss' law until time 200 for simulation with various integrators.

$$
\begin{aligned}
f(x, \boldsymbol{v}, t=0)= & \frac{1}{2 \pi \sigma^{2}} \exp \left(-\frac{v_{1}^{2}}{2 \sigma^{2}}\right)\left(\delta \exp \left(-\frac{\left(v_{2}-v_{0,1}\right)^{2}}{2 \sigma^{2}}\right)\right. \\
& \left.+(1-\delta) \exp \left(-\frac{\left(v_{2}-v_{0,2}\right)^{2}}{2 \sigma^{2}}\right)\right), \\
B_{3}(x, t=0)= & \beta \sin (k x), \\
E_{2}(x, t=0)= & 0,
\end{aligned}
$$

and $E_{1}(x, t=0)$ is computed from Poisson's equation.

We set the parameters to the following values $\sigma=0.1 / \sqrt{2}, k=0.2, \beta=-10^{-3}$, $v_{0,1}=0.5, v_{0,2}=-0.1$ and $\delta=1 / 6$. The parameters are chosen as in the case 2 of Cheng et al. (2014c). The growth rate of energy of the second component of the electric field was determined to be 0.03 in Califano et al. (1997). In figure 3, we show the electric and magnetic energies together with the analytic growth rate. We see that the growth rate is verified in the numerical solution. This simulation was performed on the domain $x \in[0,2 \pi / k)$ with 20000000 particles, 128 grid points, splines of degree 3 and 2 and $\Delta t=0.01$. Observe that the energy of the $E_{1}$ component of the electric field starts to increase at times earlier than in Califano et al. (1997), which is caused by particle noise.

As for the Weibel instability, we compare the conservation properties in table 2 for various integrators. Again we see that the Hamiltonian splitting conserves Gauss' law as opposed to the Boris-Yee scheme. The energy conservation properties of the various schemes show approximately the same behaviour as in the previous test case (see also figure 4 for the time evolution of the energy error).

\subsection{Strong Landau damping}

Finally, we also study the electrostatic example of strong Landau damping with initial distribution

$$
f(x, \boldsymbol{v})=\exp \frac{1}{2 \pi \sigma^{2}}\left(-\frac{v_{1}^{2}+v_{2}^{2}}{2 \sigma^{2}}\right)(1+\alpha \cos (k x)), \quad x \in[0,2 \pi / k), \boldsymbol{v} \in \mathbb{R}^{2} .
$$

The physical parameters are chosen as $\sigma=1, k=0.5, \alpha=0.5$ and the numerical parameters as $\Delta t=0.05, n_{x}=32$ and 100000 particles. The fields $B_{3}$ and $E_{2}$ are initialized to zero and remain zero over time. In this example, we essentially solve the Vlasov-Ampère equation with results equivalent to the Vlasov-Poisson equations. In figure 5 we show the time evolution of the electric energy associated with $E_{1}$. 


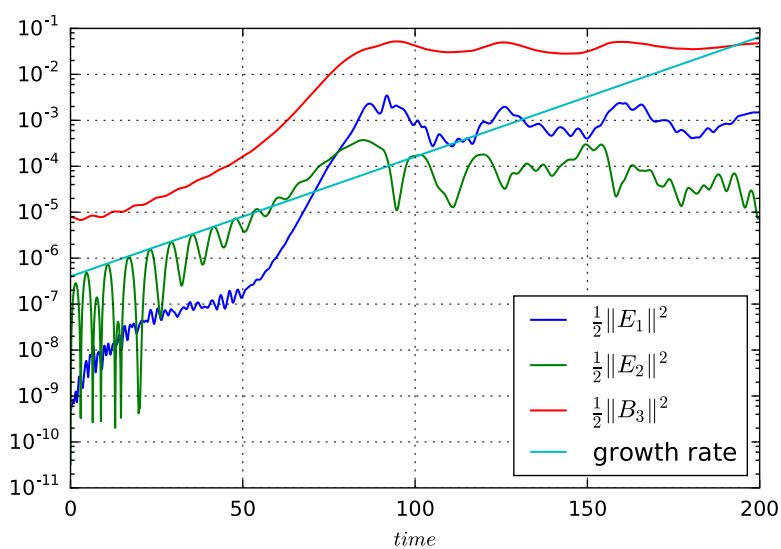

FIGURE 3. Streaming Weibel instability: the two electric and the magnetic energies together with the analytic growth rate.

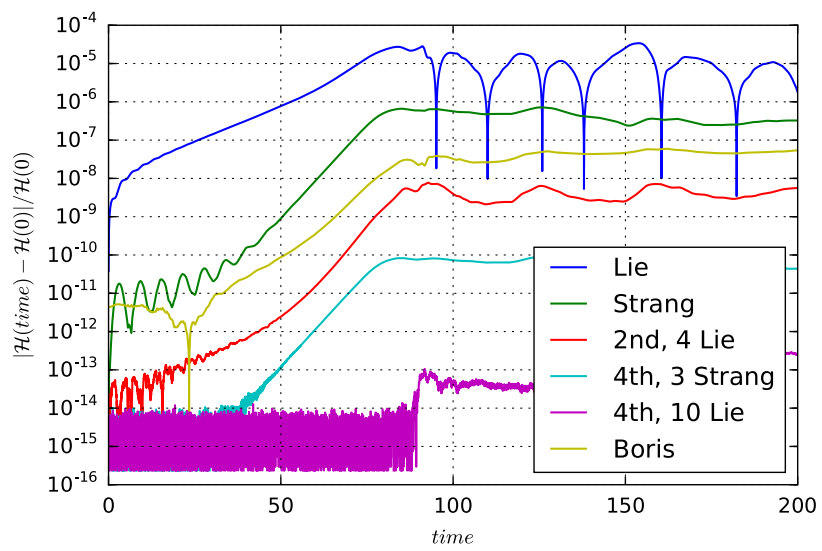

FIGURE 4. Streaming Weibel instability: difference of total energy and its initial value as a function of time for various integrators.

Integrator

$\gamma_{1}$

$-0.286+0.087$

GEMPIC

viVlasov1D (Kraus, Maj \& Sonnendruecker in preparation)

Cheng \& Knorr (1976)

Nakamura \& Yabe (1999)

de Dios \& Hajian (2012)

Heath et al. (2012)

Cheng et al. (2013)
$-0.286+0.085$

$-0.281+0.084$

$-0.280+0.085$

$-0.292+0.086$

$-0.287+0.075$

$-0.291+0.086$

TABLE 3. Damping and growth rates for strong Landau damping.

We have also fitted a damping and growth rate (using the marked local maxima in the plot). These are in good agreement with other codes (see table 3). Again 


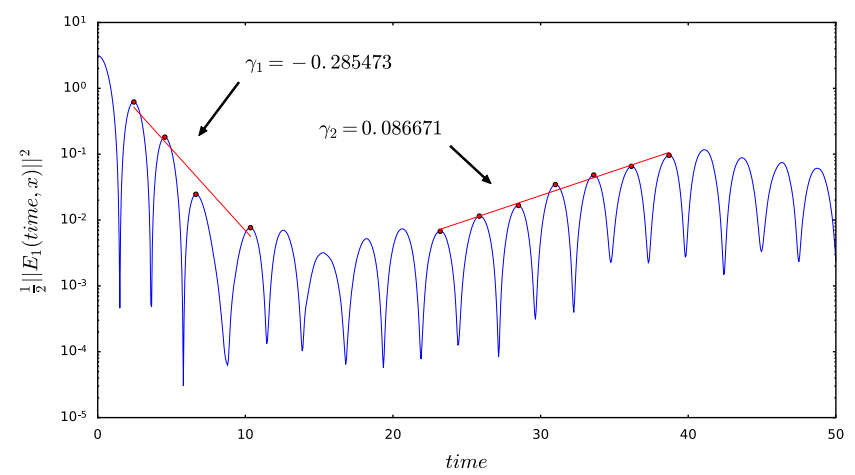

FIGURE 5. Landau damping: electric energy with fitted damping and growth rates.

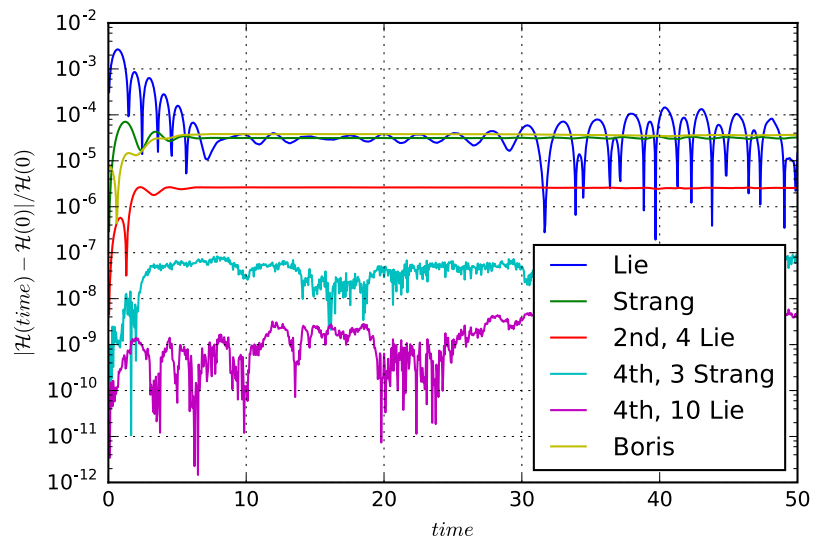

FIGURE 6. Landau damping: total energy error.

the energy conservation for the various method is visualized as a function of time in figure 6. And again we see that the fourth-order methods give excellent energy conservation.

\subsection{Backward error analysis}

For the Lie-Trotter splitting, the error in the Hamiltonian $H$ is of order $\Delta t$. However, using backward error analysis (cf. §5.3), modified Hamiltonians can be computed, which are preserved to higher order. Accounting for first-order corrections $\tilde{H}_{1}$, the error in the modified Hamiltonian,

$$
\tilde{H}=H+\Delta t \tilde{H}_{1}+O\left(\Delta t^{2}\right)
$$

is of order $(\Delta t)^{2}$. For the $1 \mathrm{~d} 2 \mathrm{v}$ example, this correction is obtained as

$$
\tilde{H}_{1}=\frac{1}{2}\left[\left\{H_{D}+H_{E}, H_{B}\right\}+\left\{H_{p_{1}}, H_{p_{2}}\right\}+\left\{H_{D}+H_{E}+H_{B}, H_{p_{1}}+H_{p_{2}}\right\}\right],
$$




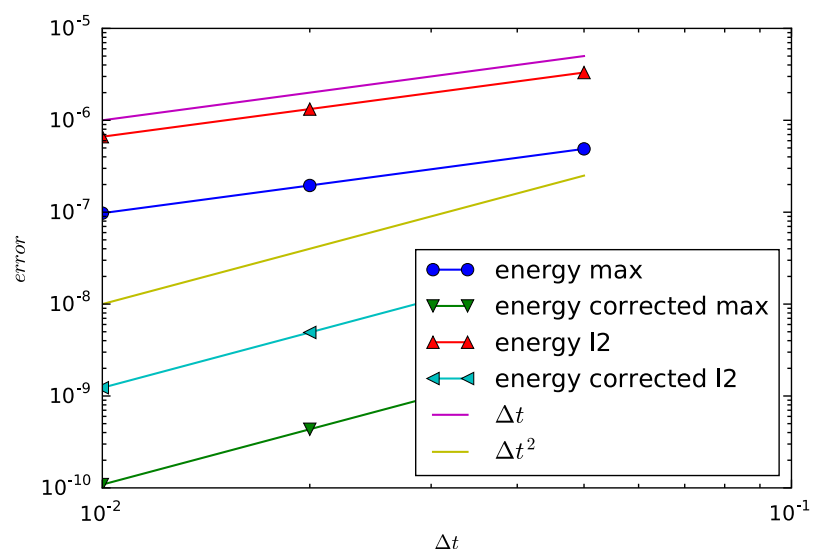

FIGURE 7. Weibel instability: error (maximum norm and $\ell_{2}$ norm) in the total energy for simulations with $\Delta t=0.01,0.02,0.05$.

with the various Poisson brackets computed as

$$
\begin{aligned}
\left\{H_{D}, H_{B}\right\} & =0, \\
\left\{H_{E}, H_{B}\right\} & =\boldsymbol{e}^{\mathrm{T}} \mathbb{C}^{\mathrm{T}} \boldsymbol{b}, \\
\left\{H_{p_{1}}, H_{p_{2}}\right\} & =\boldsymbol{V}_{1} \boldsymbol{M}_{q} \mathbb{B}(\boldsymbol{b}, \boldsymbol{X})^{\mathrm{T}} \boldsymbol{V}_{2}, \\
\left\{H_{D}, H_{p_{1}}\right\} & =-\boldsymbol{d}^{\mathrm{T}} \boldsymbol{\Lambda}^{1}(\boldsymbol{X})^{\mathrm{T}} \boldsymbol{M}_{q} \boldsymbol{V}_{1}, \\
\left\{H_{D}, H_{p_{2}}\right\} & =0 \\
\left\{H_{E}, H_{p_{1}}\right\} & =0 \\
\left\{H_{E}, H_{p_{2}}\right\} & =-\boldsymbol{e}^{\mathrm{T}} \boldsymbol{\triangle}^{0}(\boldsymbol{X})^{\mathrm{T}} \boldsymbol{M}_{q} \boldsymbol{V}_{2}, \\
\left\{H_{B}, H_{p_{1}}\right\} & =0 \\
\left\{H_{B}, H_{p_{2}}\right\} & =0
\end{aligned}
$$

In figure 7 , we show the maximum and $\ell_{2}$ error of the energy and the corrected energy for the Weibel instability test case with the parameters in $\S 7.1$. The simulations were performed with 100000 particles, 32 grid points, splines of degree 3 and 2 and $\Delta t=$ $0.01,0.02,0.05$. We can see that the theoretical convergence rates are verified in the numerical experiments. Figure 8 shows the energy as well as the modified energy for the Weibel instability test case simulated with a time step of 0.05 .

\subsection{Momentum conservation}

Finally, let us discuss conservation of momentum for our one species $1 \mathrm{~d} 2 \mathrm{v}$ equations. In this case, equation (2.30) becomes

$$
\frac{\mathrm{d} P_{e, 1,2}}{\mathrm{~d} t}=-\int E_{1,2}(x, t) \mathrm{d} x .
$$

For $P_{e, 1}(t)$ from (6.4) we get

$$
\frac{\mathrm{d}}{\mathrm{d} t} \int E_{1}(x, t) \mathrm{d} x=-\int j_{1}(x, t) \mathrm{d} x .
$$




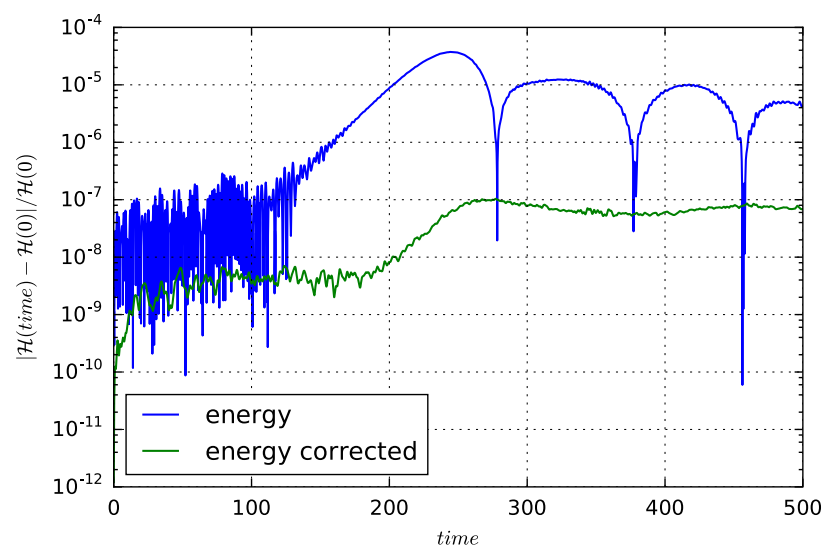

FIGURE 8. Weibel instability: energy and first-order corrected energy for simulation with $\Delta t=0.05$.

Since in general $\int j_{1}(x, t) \mathrm{d} x \neq 0$, momentum is not conserved. As a means of testing momentum conservation, Crouseilles et al. (2015) replaced (6.4) by

$$
\frac{\partial E_{1}(x, t)}{\partial t}=-j_{1}(x, t)+\int j_{1}(x, t) \mathrm{d} x .
$$

Thus, for this artificial dynamics, momentum will be conserved if $\int E_{1}(x, 0) \mathrm{d} x=0$. Instead, we do not modify the equations but check the validity of (7.12). We define a discrete version of (7.12), integrated over time, in the following way:

$$
\begin{aligned}
& \tilde{P}_{e, 1}^{n}=P_{e, 1}^{0}-\frac{\Delta t}{2} \sum_{m=1}^{n}\left(\int D_{h}^{m-1}(x) \mathrm{d} x+\int D_{h}^{m}(x) \mathrm{d} x\right), \\
& \tilde{P}_{e, 2}^{n}=P_{e, 2}^{0}-\frac{\Delta t}{2} \sum_{m=1}^{n}\left(\int E_{h}^{m-1}(x) \mathrm{d} x+\int E_{h}^{m}(x) \mathrm{d} x\right) .
\end{aligned}
$$

Our numerical scheme does not conserve momentum exactly. However, the error in momentum can be kept rather small during the linear phase of the simulation. Note that in all our examples, $\int j_{1,2}(x, t) \mathrm{d} x=0$. For a Gaussian initial distribution, the antithetic sampling ensures that $\int j_{1,2}(x, t) \mathrm{d} x=0$ holds in the discrete sense. Figure 9 shows the momentum error in a simulation of the Weibel instability as considered in $\S 7.1$, but up to time 2000 and with 25600 particles sampled from pseudo-random numbers and Sobol numbers, for both plain and antithetical sampling. For the plain sampling, momentum error is a bit smaller for Sobol numbers compared to pseudorandom numbers during the linear phase. For the antithetic sampling, we can see that the momentum error is very small until time 200 (linear phase). However, when nonlinear effects start to dominate, the momentum error slowly increases until it has reached the same level as the momentum error for plain Sobol number sampling. The level depends on the number of particles (cf. table 4). Note that the sampling does not seem to have an influence on the energy conservation as can be seen in figure 10 that compares the energy error for the various sampling techniques. The curves show that the energy error is related to the increase in potential energy during the linear phase but does not further grow during the nonlinear phase. 


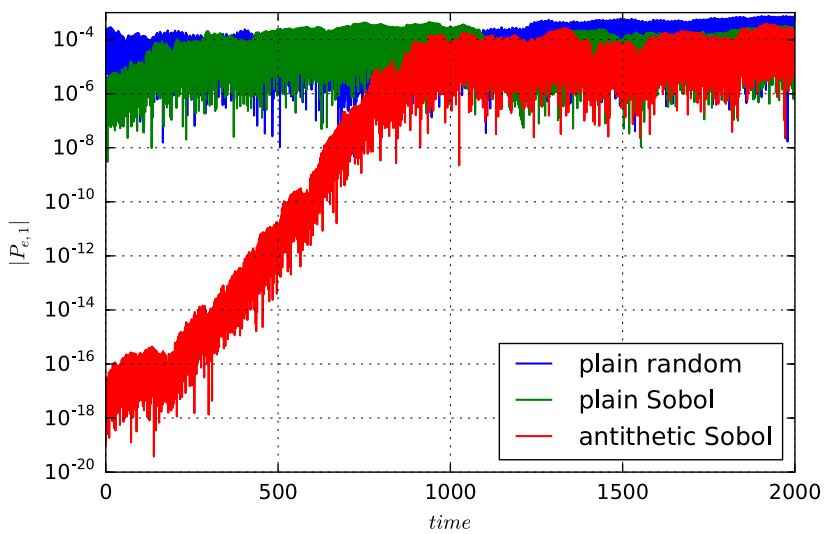

FIGURE 9. Weibel instability: error in the first component of the momentum for plain and antithetic Sobol sampling.

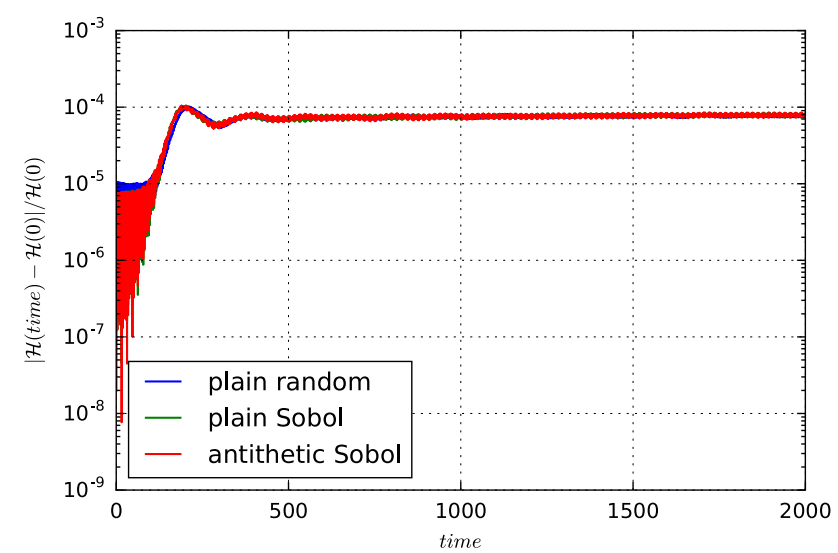

FIGURE 10. Weibel instability: total energy error for plain and antithetic Sobol sampling.

$\begin{array}{ccc}\boldsymbol{N}_{p} & P_{e, 1} & P_{e, 2} \\ 320 & 1.92 \times 10^{-2} & 1.44 \times 10^{-2} \\ 3200 & 7.17 \times 10^{-3} & 8.96 \times 10^{-3} \\ 25600 & 4.45 \times 10^{-4} & 1.49 \times 10^{-3} \\ 100000 & 1.72 \times 10^{-4} & 9.02 \times 10^{-4}\end{array}$

TABLE 4. Weibel instability: maximum error in both components of the momentum for simulations until time 2000 with various numbers of particles and 32 grid cells.

For the streaming Weibel instability on the other hand, we have a sum of two Gaussians in the second component of the velocity. Since in our sampling method we draw the particles based on Sobol quasi-random numbers, the fractions drawn from each of the Gaussians are not exactly given by $\delta$ and $1-\delta$ as in (7.5). Hence $\int j_{2}(x, t) \mathrm{d} x$ is small but non-zero. Comparing the discrete momentum defined by (7.16) 
with the discretization of its definition (2.25),

$$
P_{e, 2}^{n}=\sum_{a} v_{a, 2}^{n} m_{a} w_{a}-\int D_{h}^{n}(x) B_{h}^{n}(x) \mathrm{d} x,
$$

we see a maximum deviation over time of $1.6 \times 10^{-16}$ for a simulation with Strang splitting, 20000000 particles, 128 grid points and $\Delta t=0.01$. This shows that our discretization with a Strang splitting conserves (7.12) in the sense of (7.16) to very high accuracy.

\section{Summary}

In this work, a general framework for geometric finite element particle-in-cell methods for the Vlasov-Maxwell system was presented. The discretization proceeded in two steps. First, a semi-discretization of the noncanonical Poisson bracket was obtained, which preserves the Jacobi identity and important Casimir invariants, so that the resulting finite-dimensional system is still Hamiltonian. Then, the system was discretized in time by Hamiltonian splitting methods, still retaining exact conservation of Casimirs, which in practice means exact conservation of Gauss' law and $\operatorname{div} B=0$. Therefore the resulting method corresponds to one of the rare instances of a genuine Poisson integrator. Energy is not preserved exactly, but backward error analysis showed that the energy error does not depend on the degrees of freedom, the number of particles or the number of time steps. The favourable properties of the method were verified in various numerical experiments in $1 \mathrm{~d} 2 \mathrm{v}$ using splines as basis functions for the electromagnetic fields. One of the advantages of our approach is that conservation laws such as those for energy and charge are not manufactured into the scheme 'by hand' but follow automatically from preserving the underlying geometric structure of the equations.

The basic structure and implementation strategy of the code is very similar to existing finite element particle-in-cell methods for the Vlasov-Maxwell system. The main difference is the use of basis functions of mixed polynomial degree for the electromagnetic fields. The particle pusher is very similar to usual schemes, such as the Boris scheme, the only additional complexity being the exact computation of some line integrals. The cost of the method is comparable to existing charge-conserving algorithms like the method of Villasenor \& Buneman or the Boris correction method. It is somewhat more expensive than non-charge-conserving methods, but such schemes are known to be prone to spurious instabilities that can lead to unphysical simulation results. Even though only examples in $1 \mathrm{~d} 2 \mathrm{v}$ were shown, there are no conceptional differences or difficulties when going from one to two or to three spatial dimensions. The building blocks of the code are identical in all three cases due to the tensor-product structure of the Eulerian grid and the splitting in time. Details on the implementation of a three-dimensional version of the code as well as a comparison with existing methods will be disseminated in a subsequent publication.

The generality of the framework opens up several new paths for subsequent research. Instead of splines, other finite element spaces that form a deRham complex could be used, e.g. mimetic spectral elements or Nédélec elements for 1-forms and Raviart-Thomas elements for two-forms. Further, it also should be possible to apply this approach to other systems like the gyrokinetic Vlasov-Maxwell system (Burby et al. 2015; Burby 2017), although in this case the necessity for new splitting schemes or other time integration strategies might arise. Energy-preserving time 
stepping methods might provide an alternative to Hamiltonian splitting algorithms, where a suitable splitting cannot be easily found. This is a topic currently under investigation. So is the treatment of the relativistic Vlasov-Maxwell system, which follows very closely along the lines of the non-relativistic system. This is a problem of interest in its own right, featuring an even larger set of invariants one should attempt to preserve in the discretization. Another extension of the GEMPIC framework under development is the inclusion of non-ideal, non-Hamiltonian effects, most importantly collisions. An appropriate geometric description for such effects can be provided either microscopically by stochastic Hamiltonian processes or macroscopically by metriplectic brackets (Morrison 1986).

\section{Acknowledgements}

Useful discussions with Omar Maj and Marco Restelli are gratefully appreciated. M.K. has received funding from the European Union's Horizon 2020 research and innovation programme under the Marie Sklodowska-Curie grant agreement No 708124. P.J.M. received support from the US Dept. of Energy Contract \#DE-FG05-80ET-53088 and a Forschungspreis from the Humboldt Foundation. This work has been carried out within the framework of the EUROfusion Consortium and has received funding from the Euratom research and training programme 2014-2018 under grant agreement no. 633053. The views and opinions expressed herein do not necessarily reflect those of the European Commission. A part of this work was carried out using the HELIOS supercomputer system at Computational Simulation Centre of International Fusion Energy Research Centre (IFERC-CSC), Aomori, Japan, under the Broader Approach collaboration between Euratom and Japan, implemented by Fusion for Energy and QST.

\section{Appendix A. The Boris-Yee scheme}

As an alternative discretization scheme, we consider a Boris-Yee scheme (Yee 1966; Boris 1970) with our conforming finite elements. The scheme uses a time staggering working with the variables $\boldsymbol{X}^{n+1 / 2}=\boldsymbol{X}\left(t^{n}+\Delta t / 2\right), \boldsymbol{d}^{n+1 / 2}=\boldsymbol{d}\left(t^{n}+\Delta t / 2\right), \boldsymbol{e}^{n+1 / 2}=\boldsymbol{e}\left(t^{n}+\right.$ $\Delta t / 2), \boldsymbol{V}^{n}=\boldsymbol{V}\left(t^{n}\right)$, and $\boldsymbol{b}^{n}=\boldsymbol{b}\left(t^{n}\right)$ in the $n$th time step $t^{n}=t^{0}+n \Delta t$. The Hamiltonian at time $t^{n}$ is defined as

$$
\begin{aligned}
H= & \frac{1}{2}\left(\boldsymbol{V}_{1}^{n}\right)^{\mathrm{T}} \boldsymbol{M}_{p} \boldsymbol{V}_{1}^{n}+\frac{1}{2}\left(\boldsymbol{V}_{2}^{n}\right)^{\mathrm{T}} \boldsymbol{M}_{p} \boldsymbol{V}_{2}^{n}+\frac{1}{2}\left(\boldsymbol{d}^{n-1 / 2}\right)^{\mathrm{T}} \mathbb{M}_{1} \boldsymbol{d}^{n+1 / 2} \\
& +\frac{1}{2}\left(\boldsymbol{e}^{n-1 / 2}\right)^{\mathrm{T}} \mathbb{M}_{0} \boldsymbol{e}^{n+1 / 2}+\frac{1}{2}\left(\boldsymbol{b}^{n}\right)^{\mathrm{T}} \mathbb{M}_{1} \boldsymbol{b}^{n}
\end{aligned}
$$

Given $\boldsymbol{X}^{n-1 / 2}, \boldsymbol{d}^{n-1 / 2}, \boldsymbol{e}^{n-1 / 2}, \boldsymbol{V}^{n-1}, \boldsymbol{b}^{n-1}$ the Vlasov-Maxwell system is propagated by the following time step:

(i) Compute $\boldsymbol{b}^{n}$ according to

$$
b_{i}^{n}=b_{i}^{n-1}-\frac{\Delta t}{\Delta x}\left(e_{i}^{n-1 / 2}-e_{i-1}^{n-1 / 2}\right) .
$$

and $\boldsymbol{b}^{n-1 / 2}=\left(\boldsymbol{b}^{n-1}+\boldsymbol{b}^{n}\right) / 2$.

(ii) Propagate $\boldsymbol{v}^{n-1} \rightarrow \boldsymbol{v}^{n}$ by equation

$$
\boldsymbol{v}_{a}^{-}=\boldsymbol{v}_{a}^{n-1}+\frac{\Delta t}{2} \frac{q_{s}}{m_{s}} \boldsymbol{E}^{n-1 / 2}\left(x_{a}^{n-1 / 2}\right),
$$




$$
\begin{aligned}
v_{a, 1}^{+} & =\frac{1-\alpha^{2}}{1+\alpha^{2}} v_{a, 1}^{-}+\frac{2 \alpha}{1+\alpha^{2}} v_{a, 2}^{-}, \\
v_{a, 2}^{+} & =\frac{-2 \alpha}{1+\alpha^{2}} v_{a, 1}^{-}+\frac{1-\alpha^{2}}{1+\alpha^{2}} v_{a, 2}^{-}, \\
\boldsymbol{v}_{a}^{n} & =\boldsymbol{v}_{a}^{+}+\frac{\Delta t}{2} \frac{q_{s}}{m_{s}} \boldsymbol{E}^{n-1 / 2}\left(x_{a}^{n-1 / 2}\right),
\end{aligned}
$$

where $\alpha=q_{a} / m_{a} \Delta t / 2 B^{n-1 / 2}\left(x^{n-1 / 2}\right)$.

(iii) Propagate $x^{n-1 / 2} \rightarrow x^{n+1 / 2}$ by

$$
x_{a}^{n+1 / 2}=x_{a}^{n-1 / 2}+\Delta t v_{a, 1}^{n}
$$

and accumulate $\boldsymbol{j}_{1}^{n}, \boldsymbol{j}_{2}^{n}$ by

$$
\begin{aligned}
& \dot{j}_{1}^{n}=\sum_{a=1}^{N_{p}} w_{a} v_{a, 1}^{n} \wedge^{1}\left(\left(x_{a}^{n-1 / 2}+x_{a}^{n+1 / 2}\right) / 2\right), \\
& \dot{j}_{2}^{n}=\sum_{a=1}^{N_{p}} w_{a} v_{a, 2}^{n} \Lambda^{0}\left(\left(x_{a}^{n-1 / 2}+x_{a}^{n+1 / 2}\right) / 2\right) .
\end{aligned}
$$

(iv) Compute $\boldsymbol{d}^{n+1 / 2}$ according to

$$
\mathbb{M}_{1} d^{n+1 / 2}=\mathbb{M}_{1} d^{n-1 / 2}-\Delta t j_{1}^{n},
$$

and $\boldsymbol{e}^{n+1 / 2}$ according to

$$
\mathbb{M}_{0} \boldsymbol{e}^{n+1 / 2}=\mathbb{M}_{0} \boldsymbol{e}^{n-1 / 2}+\frac{\Delta t}{\Delta x} \mathbb{C}^{\mathrm{T}} \boldsymbol{b}^{n}-\Delta t \boldsymbol{j}_{2}^{n}
$$

For the initialization, we sample $\boldsymbol{X}^{0}$ and $\boldsymbol{V}^{0}$ from the initial sampling distribution, set $\boldsymbol{e}^{0}, \boldsymbol{b}^{0}$ from the given initial fields and solve Poisson's equation for $\boldsymbol{d}^{0}$. Then, we compute $\boldsymbol{X}^{1 / 2}, \boldsymbol{d}^{1 / 2}$ and $\boldsymbol{e}^{1 / 2}$ from the corresponding equations of the Boris-Yee scheme for a half time step, using $\boldsymbol{b}^{0}, \boldsymbol{V}^{0}$ instead of the unknown values at time $\Delta t / 4$. Note that the error in this step is of order $(\Delta t)^{2}$. But since we only introduce this error in the first time step, the overall scheme is still of order two.

\section{REFERENCES}

Arnold, D. N., FAlK, R. S. \& Winther, R. 2006 Finite element exterior calculus, homological techniques, and applications. Acta Numerica 15, 1-155.

ARnold, D. N., FALK, R. S. \& Winther, R. 2010 Finite element exterior calculus: from hodge theory to numerical stability. Bull. Am. Math. Soc. 47, 281-354.

BACK, A. \& SonnendrüCKer, E. 2014 Finite element Hodge for spline discrete differential forms. Application to the Vlasov-Poisson system. Appl. Numer. Maths 79, 124-136.

Baez, J. \& Muniain, J. P. 1994 Gauge Fields, Knots and Gravity. World Scientific.

BARThelmé, R. \& PARZAni, C. 2005 Numerical charge conservation in particle-in-cell codes. In Numerical Methods for Hyperbolic and Kinetic Problems, pp. 7-28. European Mathematical Society.

Boffi, D. 2006 Compatible discretizations for eigenvalue problems. In Compatible Spatial Discretizations, pp. 121-142. Springer. 
Boffi, D. 2010 Finite element approximation of eigenvalue problems. Acta Numerica 19, 1-120.

BoRIS, J. P. 1970 Relativistic plasma-simulation - optimization of a hybrid code. In Proceedings of the Fourth Conference on Numerical Simulation of Plasmas, pp. 3-67. Naval Research Laboratory.

Bossavit, A. 1990 Differential geometry for the student of numerical methods in electromagnetism. Lecture Notes. Available online on http://butler.cc.tut.fi/ bossavit/Books/DGSNME/DGSNME.pdf.

Bossavit, A. 2006 Applied differential geometry. Lecture Notes. Available online on http://butler.cc.tut.fi/ bossavit/BackupICM/Compendium.html.

Buffa, A. \& Perugia, I. 2006 Discontinuous Galerkin approximation of the Maxwell eigenproblem. SIAM J. Numer. Anal. 44, 2198-2226.

Buffa, A., Rivas, J., Sangalli, G. \& VÁzquez, R. 2011 Isogeometric discrete differential forms in three dimensions. SIAM J. Numer. Anal. 49, 818-844.

Buffa, A., SAngalli, G. \& VÁzQuez, R. 2010 Isogeometric analysis in electromagnetics: B-splines approximation. Comput. Meth. Appl. Mech. Engng 199 (17), 1143-1152.

Burby, J. W. 2017 Finite-dimensional collisionless kinetic theory. Phys. Plasmas 24 (3), 032101.

Burby, J. W., Brizard, A. J., Morrison, P. J. \& QIn, H. 2015 Hamiltonian Gyrokinetic VlasovMaxwell system. Phys. Lett. A 379 (36), 2073-2077.

Califano, F., Pegoraro, F. \& Bulanov, S. V. 1997 Spatial structure and time evolution of the weibel instability in collisionless inhomogeneous plasmas. Phys. Rev. E 56, 963-969.

Campos Pinto, M., Jund, S., Salmon, S. \& Sonnendrücker, E. 2014 Charge conserving FEM-PIC schemes on general grids. C. R. Méc 342 (10-11), 570-582.

Caorsi, S., Fernandes, P. \& Raffetto, M. 2000 On the convergence of Galerkin finite element approximations of electromagnetic eigenproblems. SIAM J. Numer. Anal. 38, 580-607.

Cendra, H., Holm, D. D., Hoyle, M. J. W. \& Marsden, J. E. 1998 The Maxwell-Vlasov Equations in Euler-Poincaré Form. J. Math. Phys. 39, 3138-3157.

Chandre, C., Guillebon, L., Back, A., Tassi, E. \& Morrison, P. J. 2013 On the use of projectors for Hamiltonian systems and their relationship with Dirac brackets. J. Phys. A 46, 125203.

Channell, P. J. \& Scovel, C. 1990 Symplectic integration of Hamiltonian systems. Nonlinearity 3 (2), 231-259.

Cheng, C.-Z. \& KNORR, G. 1976 The integration of the Vlasov equation in configuration space. J. Comput. Phys. 22, 330-351.

Cheng, Y., Christlieb, A. J. \& Zhong, X. 2014a Energy-conserving discontinuous Galerkin methods for the Vlasov-Ampère system. J. Comput. Phys. 256, 630-655.

Cheng, Y., Christlieb, A. J. \& ZHONG, X. $2014 b$ Energy-conserving discontinuous Galerkin methods for the Vlasov-Maxwell system. J. Comput. Phys. 279, 145-173.

Cheng, Y., Gamba, I. M., LI, F. \& Morrison, P. J. 2014c Discontinuous Galerkin methods for the Vlasov-Maxwell equations. SIAM J. Numer. Anal. 52 (2), 1017-1049.

Cheng, Y., Gamba, I. M. \& Morrison, P. J. 2013 Study of conservation and recurrence of Runge-Kutta discontinuous Galerkin schemes for Vlasov-Poisson systems. J. Sci. Comput. 56, 319-349.

Christiansen, S. H., Munthe-KaAs, H. Z. \& Owren, B. 2011 Topics in structure-preserving discretization. Acta Numerica 20, 1-119.

Crouseilles, N., Einkemmer, L. \& FAoU, E. 2015 Hamiltonian splitting for the Vlasov-Maxwell equations. J. Comput. Phys. 283, 224-240.

Crouseilles, N., Navaro, P. \& Sonnendrücker, E. 2014 Charge conserving grid based methods for the Vlasov-Maxwell equations. C. R. Méc 342 (10-11), 636-646.

Darling, R. W. R. 1994 Differential Forms and Connections. Cambridge University Press.

DE Dios, B. A., Carrillo, J. \& ShU, C.-W. 2011 Discontinuous Galerkin methods for the onedimensional Vlasov-Poisson system. Kinet. Relat. Models 4, 955-989.

De Dios, B. A., Carrillo, J. A. \& ShU, C.-W. 2012 Discontinuous Galerkin methods for the multi-dimensional vlasov-poisson problem. Math. Models Meth. Appl. Sci. 22, 1250042. 
DE Dios, B. A. \& HaJiAn, S. High order and energy preserving discontinuous Galerkin methods for the Vlasov-Poisson system, Preprint, 2012, arXiv:1209.4025.

Desbrun, M., Kanso, E. \& Tong, Y. 2008 Discrete differential geometry. In Discrete Differential Forms for Computational Modeling, pp. 287-324. Birkhäuser, ISBN 978-3-7643-8621-4.

DRAY, T. 2014 Differential Forms and the Geometry of General Relativity. CRC Press.

EAstwood, J. W. 1991 The virtual particle electromagnetic particle-mesh method. Comput. Phys. Commun. 640 (2), 252-266.

ESIRKEPOV, T. Z. 2001 Exact charge conservation scheme for particle-in-cell simulation with an arbitrary form-factor. Comput. Phys. Commun. 135 (2), 144-153.

Evstatiev, E. G. \& Shadwick, B. A. 2013 Variational formulation of particle algorithms for kinetic plasma simulations. J. Comput. Phys. 245, 376-398.

Gerritsma, M. 2012 An Introduction to a compatible spectral discretization method. Mech. Adv. Mater. Struct. 19, 48-67.

Hairer, E., Lubich, C. \& Wanner, G. 2006 Geometric Numerical Integration. Springer.

He, Y., QIN, H., Sun, Y., XiaO, J., Zhang, R. \& LiU, J. 2015 Hamiltonian integration methods for Vlasov-Maxwell equations. Phys. Plasmas 22, 124503.

HE, Y., Sun, Y., QIN, H. \& LIU, J. 2016 Hamiltonian particle-in-cell methods for Vlasov-Maxwell equations. Phys. Plasmas 23 (9), 092108.

Heath, R. E., Gamba, I. M., Morrison, P. J. \& Michler, C. 2012 A discontinuous Galerkin method for the Vlasov-Poisson system. J. Comput. Phys. 231, 1140-1174.

Hesthaven, J. S. \& Warburton, T. 2004 High-order nodal discontinuous Galerkin methods for the Maxwell eigenvalue problem. Phil. Trans. R. Soc. Lond. A 362 (1816), 493-524.

Hirani, A. N. 2003 Discrete exterior calculus. PhD thesis, California Institute of Technology, http://resolver.caltech.edu/CaltechETD:etd-05202003-095403.

Holloway, J. P. 1996 On numerical methods for Hamiltonian PDEs and a collocation method for the Vlasov-Maxwell equations. J. Comput. Phys. 129 (1), 121-133.

Kraus, M. 2013 Variational integrators in plasma physics. PhD thesis, Technische Universität München, arXiv:1307.5665.

Kraus, M., Maj, O. \& SonnendrüCKer, E. Variational integrators for the Vlasov-Poisson system. (in preparation).

Kreeft, J., Palha, A. \& Gerritsma, M. Mimetic framework on curvilinear quadrilaterals of arbitrary order, Preprint, 2011, arXiv:1111:4304.

LAngdon, A. B. 1992 On enforcing Gauss' law in electromagnetic particle-in-cell codes. Comput. Phys. Commun. 70, 447-450.

LEWIS, H. R. 1970 Energy-conserving numerical approximations for Vlasov plasmas. J. Comput. Phys. 6 (1), 136-141.

LEWIS, H. R. 1972 Variational algorithms for numerical simulation of collisionless plasma with point particles including electromagnetic interactions. J. Comput. Phys. 10 (3), 400-419.

Low, F. E. 1958 A Lagrangian formulation of the Boltzmann-Vlasov equation for plasmas. Proc. R. Soc. Lond. A 248, 282-287.

Madaule, É., Restelli, M. \& Sonnendrücker, E. 2014 Energy conserving discontinuous Galerkin spectral element method for the Vlasov-Poisson system. J. Comput. Phys. 279, 261-288.

MARDER, B. 1987 A method for incorporating Gauss's law into electromagnetic PIC codes. J. Comput. Phys. 68, 48-55.

Marsden, J. E. \& Weinstein, A. 1982 The Hamiltonian structure of the Maxwell-Vlasov equations. Physica D 4 (3), 394-406.

MCLACHLAN, R. I. 1995 On the numerical integration of ordinary differential equations by symmetric composition methods. SIAM J. Sci. Comput. 16 (1), 151-168.

McLachlan, R. I. \& Quispel, G. R. W. 2002 Splitting methods. Acta Numerica 11, 341-434.

Monk, P. 2003 Finite Element Methods for Maxwell's Equations. Oxford University Press.

Moon, H., TeiXeira, F. L. \& OMelchenko, Y. A. 2015 Exact charge-conserving scatter-gather algorithm for particle-in-cell simulations on unstructured grids: a geometric perspective. Comput. Phys. Commun. 194, 43-53. 
Moore, B. E. \& ReICH, S. 2003 Multi-symplectic integration methods for Hamiltonian PDEs. Future Generation Comput. Syst. 19 (3), 395-402.

Morita, S. 2001 Geometry of Differential Forms. American Mathematical Society.

Morrison, P. J. 1980 The Maxwell-Vlasov equations as a continuous Hamiltonian system. Phys. Lett. A 80 (5-6), 383-386.

MORRISON, P. J. 1981a Hamiltonian field description of the one-dimensional Poisson-Vlasov equations. Tech. Rep. PPPL-1788, Princeton Plasma Physics Laboratory.

MORRISON, P. J. $1981 b$ Hamiltonian field description of the two-dimensional vortex fluids and guiding center plasmas. Tech. Rep. PPPL-1783, Princeton Plasma Physics Laboratory.

Morrison, P. J. 1982 Poisson brackets for fluids and plasmas. In AIP Conference Proceedings, vol. 88, pp. 13-46. AIP.

Morrison, P. J. 1986 A paradigm for joined Hamiltonian and dissipative systems. Physica D 18, $410-419$.

MORRISON, P. J. 1987 Variational principle and stability of nonmonotonic Vlasov-Poisson equilibria. Z. Naturforsch. A 42, 1115-1123.

Morrison, P. J. 1998 Hamiltonian description of the ideal fluid. Rev. Mod. Phys. 70, 467-521.

Morrison, P. J. A general theory for gauge-free lifting. Phys. Plasmas 20 (1), 012104.

Morrison, P. J. \& PFirsch, D. 1989 Free-energy expressions for Vlasov equilibria. Phys. Rev. A 40, 3898-3910.

Munz, C.-D., Omnes, P., Schneider, R., Sonnendrücker, E. \& Voss, U. 2000 Divergence correction techniques for Maxwell solvers based on a hyperbolic model. J. Comput. Phys. 161, 484-511.

Munz, C.-D., Schneider, R., Sonnendrücker, E. \& Voß, U. 1999 Maxwell's equations when the charge conservation is not satisfied. C. R. Acad. Sci. I 328 (5), 431-436.

NAKAMURA, T. \& YABE, T. 1999 Cubic interpolated propagation scheme for solving the hyperdimensional Vlasov-Poisson equation in phase space. J. Comput. Phys. 120, 122-154.

Palha, A., Rebelo, P. P., Hiemstra, R., Kreeft, J. \& Gerritsma, M. 2014 Physics-compatible discretization techniques on single and dual grids, with application to the Poisson equation of volume forms. J. Comput. Phys. 257, 1394-1422.

Qin, H., He, Y., Zhang, R., LiU, J., XiaO, J. \& Wang, Y. 2015 Comment on Hamiltonian splitting for the Vlasov-Maxwell equations. J. Comput. Phys. 297, 721-723.

Qin, H., LiU, J., XiaO, J., Zhang, R., He, Y., Wang, Y., Sun, Y., Burby, J. W., Ellison, L. \& ZHOU, Y. 2016 Canonical symplectic particle-in-cell method for long-term large-scale simulations of the Vlasov-Maxwell equations. Nucl. Fusion 56 (1), 014001.

RAtnAni, A. \& SonNendRÜCKer, E. 2012 An arbitrary high-order spline finite element solver for the time domain Maxwell equations. J. Sci. Comput. 51 (1), 87-106.

REICH, S. 2000 Multi-symplectic Runge-Kutta collocation methods for Hamiltonian wave equations. J. Comput. Phys. 157 (2), 473-499.

SAnZ-Serna, J. M. \& CALvo, M.-P. 1993 Symplectic numerical methods for Hamiltonian problems. Intl J. Mod. Phys. C 04 (02), 385-392.

Scovel, C. \& Weinstein, A. 1994 Finite dimensional Lie-Poisson approximations to Vlasov-Poisson equations. Commun. Pure Appl. Maths 47 (5), 683-709.

SeLaLib. http://selalib.gforge.inria.fr/.

Shadwick, B. A., Stamm, A. B. \& Evstatiev, E. G. 2014 Variational formulation of macroparticle plasma simulation algorithms. Phys. Plasmas 21 (5), 055708.

Sircombe, N. J. \& Arber, T. D. 2009 VALIS: A split-conservative scheme for the relativistic 2D Vlasov-Maxwell system. J. Comput. Phys. 228, 4773-4788.

SONNENDRÜCKER, E. 2017 Numerical Methods for the Vlasov-Maxwell Equations. Springer.

SQuire, J., QIN, H. \& TANG, W. M. 2012 Geometric integration of the Vlasov-Maxwell system with a variational particle-in-cell scheme. Phys. Plasmas 19, 084501.

StAmm, A. B. \& SHADWICK, B. A. 2014 Variational formulation of macroparticle models for electromagnetic plasma simulations. IEEE Trans. Plasma Sci. 42 (6), 1747-1758.

Stern, A., Tong, Y., Desbrun, M. \& Marsden, J. E. 2014 Geometric computational electrodynamics with variational integrators and discrete differential forms. In Geometry, 
Mechanics and Dynamics: The Legacy of Jerry Marsden, Fields Institute Communications. Springer.

StRAnG, G. 1968 On the construction and comparison of difference schemes. SIAM J. Numer. Anal. 5 (3), 506-517.

SUZUKI, M. 1990 Fractal decomposition of exponential operators with applications to many-body theories and Monte Carlo simulations. Phys. Lett. A 146 (6), 319-323.

Trotter, H. F. 1959 On the product of semi-groups of operators. Proc. Am. Math. Soc. 10 (4), $545-551$.

Umeda, T., Omura, Y., Tominaga, T. \& Matsumoto, H. 2003 A new charge conservation method in electromagnetic particle-in-cell simulations. Comput. Phys. Commun. 156 (1), 73-85.

Villasenor, J. \& BUneman, O. 1992 Rigorous charge conservation for local electromagnetic field solvers. Comput. Phys. Commun. 69 (2), 306-316.

WARnick, K. F. \& RusSER, P. H. 2006 Two, three and four-dimensional electromagnetics using differential forms. Turkish J. Elect. Engng Comput. Sci. 14 (1), 153-172.

WARnick, K. F., Selfridge, R. H. \& ARnOld, D. V. 1998 Teaching electromagnetic field theory using differential forms. IEEE Trans. Edu. 1, 53-68.

WeIBEL, E. S. 1959 Spontaneously growing transverse waves in a plasma due to an anisotropic velocity distribution. Phys. Rev. Lett. 2, 83-84.

Weinstein, A. \& Morrison, P. J. 1981 Comments on: the Maxwell-Vlasov equations as a continuous Hamiltonian system. Phys. Lett. A 86 (4), 235-236.

XiaO, J., QIn, H., LiU, J., He, Y., Zhang, R. \& SUn, Y. 2015 Explicit high-order non-canonical symplectic particle-in-cell algorithms for Vlasov-Maxwell systems. Phys. Plasmas 22, 112504.

Ye, H. \& Morrison, P. J. 1992 Action principles for the Vlasov equation. Phys. Fluids B 4 (4), 771-777.

YEE, K. S. 1966 Numerical solution of initial boundary value problems involving Maxwell's equations in isotropic media. IEEE Trans. Antennas Propag. 14, 302-307.

Yoshida, H. 1990 Construction of higher order symplectic integrators. Phys. Lett A 150 (5-7), 262-268.

Yu, J., Jin, X., Zhou, W., LI, B. \& GU, Y. 2013 High-order interpolation algorithms for charge conservation in particle-in-cell simulations. Commun. Comput. Phys. 13, 1134-1150. 